\title{
Compensatory CSF2-driven macrophage activation promotes adaptive resistance to CSF1R inhibition in breast-to-brain metastasis
}

Florian Klemm ${ }^{1,2}$, Alexander Schäffer ${ }^{3}$, Anna Salamero-Boix ${ }^{3,4}$, Tijna Alekseeva ${ }^{3}$, Michael Schulz ${ }^{3,4}$, Katja Niesel ${ }^{3}$, Roeltje R. Maas ${ }^{1,2,5,6}$, Marie Groth $^{7}$, Benelita T. Elie ${ }^{7}$, Robert L. Bowman $^{7}$, Monika E. Hegi ${ }^{5,6}$, Roy T. Daniel ${ }^{6}$, Pia S. Zeiner ${ }^{8,9}$, Jenny Zinke ${ }^{8}$, Patrick N. Harter ${ }^{8}$, 10, 11,12, Karl H. Plate ${ }^{8,10,11,12}$, Johanna A. Joyce ${ }^{1,2^{*}}$ and Lisa Sevenich ${ }^{3,10,11,12^{*}}$

${ }^{1}$ Department of Oncology, University of Lausanne, Lausanne, Switzerland

${ }^{2}$ Ludwig Institute for Cancer Research, Lausanne, Switzerland

${ }^{3}$ Georg-Speyer-Haus, Institute for Tumor Biology and Experimental Therapy, Frankfurt am Main, Germany

${ }^{4}$ Biological Sciences, Faculty 15, Goethe University Frankfurt, Frankfurt am Main, Germany

${ }^{5}$ Neuroscience Research Center, Centre Hospitalier Universitaire Vaudois, Lausanne,

Switzerland

${ }^{6}$ Department of Neurosurgery, Centre Hospitalier Universitaire Vaudois, Lausanne, Switzerland

${ }^{7}$ Cancer Biology and Genetics Program, Memorial Sloan Kettering Cancer Center, New York, NY, USA

${ }^{8}$ Institute of Neurology (Edinger Institute), Goethe University Hospital Frankfurt, Frankfurt am Main, Germany

${ }^{9}$ Dr. Senckenberg Institute of Neurooncology, Goethe University Frankfurt, Frankfurt am Main, Germany

${ }^{10}$ German Cancer Research Center (DKFZ), Heidelberg, Germany

${ }^{11}$ German Cancer Consortium (DKTK), Partner Site Frankfurt/Mainz, Germany

${ }^{12}$ Frankfurt Cancer Institute (FCI), Goethe University Frankfurt, Frankfurt am Main, Germany

*Correspondence: Johanna A. Joyce (johanna.joyce@unil.ch) and Lisa Sevenich (sevenich@gsh.uni-frankfurt.de) 


\section{SUMMARY}

Tumor microenvironment-targeted therapies are emerging as promising treatment options for different cancer types. Tumor-associated macrophages and microglia (TAMs) represent an abundant non-malignant cell type in brain metastases and have been proposed to modulate metastatic colonization and outgrowth. We used an inhibitor of colony stimulating factor 1 receptor (CSF1R) to target TAMs at distinct stages of the metastatic cascade in preclinical breast-to-brain metastasis models and found that CSF1R inhibition leads to anti-tumor responses in prevention and intervention trials. However, in established brain metastases, compensatory CSF2Rb-STAT5-mediated pro-inflammatory TAM activation blunted the ultimate efficacy of CSF1R inhibition by inducing neuro-inflammation gene signatures in association with wound repair responses that fostered tumor recurrence. Consequently, combined blockade of CSF1R and STAT5 signaling led to sustained tumor control, a normalization of microglial activation states and amelioration of neuronal damage. 


\section{INTRODUCTION}

Brain metastases (BrM) represent the most common intracranial tumors in adults. It is estimated that approximately $20-40 \%$ of all cancer patients develop BrM, with melanoma, breast, and lung cancer showing the highest incidence ${ }^{1-4}$. Despite recent advances in the management of extracranial primary tumors, treatment options for disseminated disease remain limited and largely depend on multimodal approaches involving surgical resection, radio- and/or chemotherapy ${ }^{5,6}$. The success of immunotherapies in several non-cerebral cancers has led to considerable optimism for the development of more efficient treatment options for brain tumors, including glioblastoma and $\mathrm{BrM}^{7,8}$. However, strategies that aim to activate $T$ cell responses directed against tumor cells have largely failed as monotherapies in primary brain tumors and showed low or moderate efficacy in BrM patients depending on the primary tumor type ${ }^{9-11}$. Low response rates can in part be attributed to the unique tissue environment of the central nervous system (CNS), which is highly immune-suppressive ${ }^{12,13}$.

Tumor-associated macrophages (TAMs) are one of the most abundant non-cancerous cell types in breast-BrM ${ }^{14-16}$ and originate from brain-resident microglia (TAM-MG) or monocytederived macrophages (TAM-MDM) ${ }^{14,15,17,18}$ which exhibit an immunomodulatory phenotype in $\mathrm{BrM}^{14}$. Therefore, targeting this highly abundant TAM population could represent a viable strategy to disrupt cancer-permissive tumor-stroma interactions and to modulate the immune response directed against cancer cells for more effective tumor control. Indeed, preclinical studies have demonstrated that TAM-targeted therapies can lead to anti-tumor efficacy in glioblastoma $^{19-21}$, melanoma-BrM ${ }^{22}$, and in the context of intracerebral injection of 4T1 breast cancer cells ${ }^{23}$. However, these previous studies interrogated the role of TAMs in BrM by depleting the cells only at single time points in the metastatic process and did not discriminate cell-type specific functions or the consequences of CSF1R blockade on TAM-MG and TAMMDM, which may have important implications for clinical translation. Thus, in order to address this knowledge gap, we used an inhibitor of colony stimulating factor 1 receptor (CSF1R) to deplete TAMs at distinct stages of BrM, to study their role in metastatic progression, and to evaluate the efficacy of TAM-targeted therapies for BrM in different preclinical trial settings.

\section{RESULTS}

CSF1R expression in human and mouse brain metastases

We first analyzed the abundance of CSF1R+ cells in patient BrM tissue arrays originating from different cancer types including small cell lung cancer (SCLC), non-small cell lung cancer (NSCLC), renal cell cancer (RCC), melanoma, colon and breast cancer (Extended data Table 1). We observed high infiltration of CSF1R+ immune cells across all analyzed BrM lesions, with RCC-BrM showing the largest proportion of CSF1R+ cells, and SCLC-BrM the lowest 
(Fig. 1a,b). Localization of CSF1R+ cells showed a considerable overlap with IBA1 expression in all analyzed biopsies, indicating that the majority of CSF1R+ cells originate from the monocyte/macrophage lineage. Further investigation in an independent cohort of breast cancer-BrM and NSCLC-BrM whole tissue sections (Fig. 1c, Extended data Fig. 1, Extended data Table 2) confirmed CSF1R expression in a significant proportion of BrM-MG and BrMMDMs, whereas very few non-immune cells were CSF1R+. To examine the potential relevance of low level CSF1R expression in tumor cells, we quantified CSF1R/Csf1r across a panel of human and mouse cancer cell lines. Quantitative real-time PCR showed either no or very low CSF1R/Csf1r expression in cancer cell lines derived from different primary tumors, compared to macrophages as a positive control (Extended data Fig. 2a,b). Moreover, CSF1R expression levels in FACS-purified tumor cells isolated from experimental BrM lesions were significantly lower than from tumor-associated MG and MDM or their normal counterparts (Extended data Fig. 2c). Finally, tumor cell viability was not affected by treatment with the CSF1R inhibitor BLZ945 in vitro (Extended data Fig. 2d), indicating that even though rare tumor cells can express low levels of CSF1R, their survival is not directly dependent on CSF1R signaling, and its inhibition rather targets myeloid cells.

\section{Ontogenetic origin and molecular identity of TAMs in BrM}

We next analyzed the localization and morphology of TAMs in the xenograft MDA-BrM and syngeneic $99 \mathrm{LN}-\mathrm{BrM} 2$ breast-BrM mouse models ${ }^{24}$. TAM activation was associated with a gradual change in cell morphology from ramified, resting $M G$ in the adjacent brain parenchyma to amoeboid, activated TAMs within the tumor lesion (Fig. 2a). Flow cytometric analysis using previously established lineage-specific cell surface markers ${ }^{17}$, revealed that the majority of TAMs in BrM were derived from brain-resident MG, while on average $7 \%$ and $17 \%$ were recruited from the periphery in the MDA-BrM and 99LN-BrM models respectively (Extended data Fig. 2e). We performed RNA sequencing (RNAseq) analyses of FACS-purified TAM-MG and TAM-MDM from MDA-BrM tumors compared to their respective control population in tumor-free animals, i.e. normal MG or blood monocytes, to gain deeper insight into the molecular identity of TAMs in BrM (Fig. 2b,c, see also Extended data Table S3). The purity of the FACS-sorted populations was further validated by the MG- and MDM-specific expression of cell type-restricted markers (Extended data Fig. 2f). RNAseq analysis revealed 966 differentially expressed genes (DEG) in the TAM-MG population compared to normal MG, and 2863 DEG for the comparison between TAM-MDM and normal monocytes; 500 of which were shared between both TAM populations (Fig. 2b and Extended data Table 3). We also queried an RNAseq dataset from matched primary breast cancer and BrM bulk tissue samples ${ }^{25}$ for the expression of the 3329 DEG identified in the comparison of TAMs to normal MG and monocytes in the mouse model. Interestingly, we found a higher enrichment of the metastasis 
TAM genes in BrM compared to the matched primary breast cancer both in triple negative breast cancer (TNBC) and in other molecular subtypes collectively denoted as non-TNBC (Extended data Fig. 2g). This suggests that these TAM-DEG are acquired in a breast BrMspecific fashion. We next performed gene ontology analysis of clustered gene groups within the 500 genes commonly enriched in both TAM populations (Fig. $2 \mathrm{c}$ and Extended data Table 4). TAM-MDM showed a higher expression of gene signatures that represent pathways associated with antigen presentation, and tissue remodeling and immune regulation (cluster 5, 6 and 8) as well as mitosis and cell division (cluster 7), while TAM-MG showed a higher enrichment of genes associated with wounding responses and regulation of neurological processes (cluster 3). Signatures associated with negative regulation of CNS development, metabolism and leukocyte recruitment (cluster 1, 2 and 4) showed similar induction in TAMMG and TAM-MDM. Taken together, our data indicate that education of TAMs in BrM leads to the induction of cancer-associated inflammation that has previously been associated with tumor promotion across different cancer types ${ }^{26-29}$. However, the functional contributions of TAM-MG and TAM-MDM at distinct stages of breast-to-brain metastasis remains largely unknown.

\section{MG support tumor cell extravasation}

We next sought to investigate the consequences for tumor cell fate after initial contact with MG. We therefore employed a MG depletion strategy using the blood-brain barrier (BBB)permeable CSF1R inhibitor BLZ945 ${ }^{19}$, and began by treating normal, non-tumor bearing mice which resulted in a 90\% decrease in MG after 7 days (Fig. 3a,b). To dynamically track the first encounter of MG and tumor cells in the brain parenchyma, we performed ex vivo brain slice assays (Fig. 3c). MG in brain slices that were isolated from vehicle-treated mice displayed directed movements towards exogenously-added 99LN-BrM cells and extended their protrusions to survey the tumor cell surface (Fig. 3d and Extended data Movie 1), consistent with their known role in immune surveillance ${ }^{30}$. The MG that remained in brain slices from BLZ945-treated animals were still motile and the formation of protrusions was visible. However, the execution of movements was less targeted, suggesting that following CSF1R inhibition MG failed to detect the presence of tumor cells (Fig. 3d and Extended data Movie 2). Indeed, quantification of tumor cell-MG interactions, normalized to the number of MG present following BLZ945 treatment, revealed a significant reduction in direct contacts (Fig. $3 e)$. We also detected fewer tumor cells after $20 \mathrm{~h}$ in culture in brain slices isolated from BLZ945-treated animals, compared to vehicle controls (Fig. 3f), suggesting that the initial tumor cell-MG interactions in the controls result in the support of tumor cell colonization rather than recognition and killing of tumor cells. 
To further evaluate if these tumor cell-MG interactions are also apparent at the BBB in vivo, we treated mice with BLZ945 or vehicle for 7 days before intracardiac injection of tumor cells and analyzed their rate of extravasation in the brain. We observed a significant delay in extravasation in BLZ945-treated 99LN-BrM animals after 24 and 48h (Fig. 3g,h). Similar results were observed in the MDA-BrM model after 48h (Fig. 3h). Consistent with the observations from the ex vivo brain slice assay with exogenously-added tumor cells, we detected fewer direct interactions between tumor cells and MG at sites of extravasation in BLZ945-treated mice, again normalized to the number of MG (Fig. 3i). In vitro BBB assays further confirmed that the addition of freshly isolated primary MG, or the MG cell line EOC2, increased the BBB transmigration potential of 99LN-BrM and MDA-BrM tumor cell lines (Extended data Fig. 3a-c), and this effect was significantly reduced by BLZ945 (Extended data Fig. 3b,c).

TAMs support metastatic colonization and outgrowth

In order to evaluate whether the impaired BBB transmigration translates into decreased BrM incidence or delayed tumor outgrowth, we followed BrM progression in response to BLZ945 treatment. CSF1R inhibition in the prevention trial setting resulted in $94 \%$ and $73 \%$ reduction of the number of lba+ cells in MDA-BrM and 99LN-BrM endpoint tumors respectively (Extended data Fig. 3d-g). We determined the time point when tumor lesions were first detectable, and evaluated effects on overall survival using the 99LN-BrM and MDA-BrM mouse models (Fig. 3j). We found a significant delay in tumor onset in mice that developed BrM in the context of BLZ945 treatment in both models (Fig. 3k,l, and Fig. 3n,o). This effect translated into significantly prolonged median survival of BLZ945-treated animals in the MDABrM model (Fig. $3 \mathrm{~m}$ ). However, once tumors developed, the growth rate was similar in vehicle and BLZ945-treated animals (Extended data Fig. 3h). We observed similar effects with a delay in tumor onset in the 99LN-BrM model, but no significant differences in median survival were detected (Fig. 3p, see also Extended data Fig. 3i). This indicates that tumor cells can ultimately compensate for the loss of supportive TAM functions after successful metastatic colonization either by tumor cell-intrinsic traits or by engaging the support of other immune or stromal cell types that are not CSF1R-dependent.

\section{CSF1R inhibition transiently blocks tumor growth in established BrM}

We subsequently evaluated the effects of CSF1R inhibition in established $\mathrm{BrM}$, and stratified tumor-bearing mice into vehicle and BLZ945 treatment groups, starting with a similar tumor volume, based on BLI output or volumetric MRI measurements. Mice were then treated once daily for 7 days or until symptoms developed. Measurements of tumor progression were 
performed on $\mathrm{d} 4$ and $\mathrm{d} 7$ after treatment initiation or once per week until the trial endpoint (Fig. 4a). Imaging by $\mathrm{BLI}$ or MRI revealed that TAM depletion leads to a blockade of tumor outgrowth in the majority of BLZ945-treated animals, with a few animals showing regression of large BrM lesions (Fig. 4b-e). Quantification of the depletion rate indicated $63 \%$ or $65 \%$ decrease of IBA1+ TAMs in MDA-BrM or 99LN-BrM respectively (Extended data Fig. 4a-d). Flow cytometry further confirmed depletion of CSF1R-expressing cell types including MG, MDM and inflammatory monocytes, while the relative abundance of granulocytes increased (Extended data Fig. 4e,g). Other tumor-associated cell types including T cells in the 99LNBrM model were not affected by CSF1R inhibition (Extended data Fig. 4f,h). Decreased tumor burden was associated with a reduction in the proliferation:apoptosis (Ki67:CC3) index in BLZ945-treated MDA-BrM and 99LN-BrM lesions (Extended data Fig. 5a-d). However, while CSF1R inhibition led to initial tumor responses at d4 and d7 after treatment initiation (Fig. 4d,e, see also Extended data Fig. 5e,f), BrM regrowth was subsequently detected in both models (Extended data Fig. 4e,f). Consequently, no significant effects on median survival in either BrM model were observed (Fig. 4f,g).

\section{TAM depletion leads to gene expression changes in tumor cells}

We next FACS-purified tumor cells from vehicle and BLZ945-treated MDA-BrM mice 7 days after treatment initiation to perform RNAseq for further mechanistic insight into indirect effects on tumor cells as a consequence of CSF1R inhibition (Fig. 5a,b). Analysis of DEG in tumor cells revealed that the down-regulated genes were predominantly associated with DNA repair, cell cycle or transcriptional regulation, e.g. XRCC2, CDK1, CCNF, TOP2A and ESCO2, or represented receptor tyrosine kinases involved in wound repair, tumor growth and invasiveness, e.g. DDR2 (Fig. 5c and Extended data Table 5). Gene ontology analysis further confirmed that gene sets related to cell cycle regulation and proliferation were found among the top 20 pathways in the gene set overrepresentation analysis (ORA, Fig. 5d) of genes downregulated in MDA-BrM upon BLZ945 treatment.

Interestingly, evaluation of the list of up-regulated DEG revealed multiple genes that are typically enriched in cells of the CNS, e.g. GPR153, or are associated with diverse CNS pathways (Fig. 5c). Among these PCDH2O, BTBD6 and TMEM158 are known to be involved in CNS cell-cell communication, neuronal differentiation, or neuronal survival, respectively ${ }^{31-}$ ${ }^{34}$. Gene set enrichment analysis (GSEA) additionally demonstrated expression changes in genes annotated as 'Regulation of Neuron Differentiation' (G00045666) and 'Cell Morphogenesis involved in Neuron Differentiation' (GO0048812) in tumor cells following in vivo BLZ945 treatment (Fig. 5e). Histological evaluation confirmed higher expression of GPR153, PCDH20 and TMEM158 in tumor cells in BLZ945-treated animals in the 99LN-BrM 
model (Fig. 5f). Together these data suggest a form of neuronal mimicry, which may represent a general trait of brain metastatic tumor cells to integrate into CNS cell-cell communication circuits that support cell survival- in this case, as an adaptation to TAM depletion.

\section{CSF2Rb-STAT5 downstream signaling compensates for CSF1R inhibition in TAMs}

To gain functional insight into the direct effects of CSF1R inhibition on TAMs, we FACSpurified TAM-MG and TAM-MDM from vehicle and BLZ945-treated animals (Extended data Fig. 6a-e, Extended data Table 3), as well as MG and blood monocytes from normal mice as controls for RNAseq analyses. We first queried the expression of putative M1-like and M2-like genes ${ }^{35}$ and found that TAMs acquire a mixed phenotype in BrM based on expression of polarization markers and transcription factor activity (Fig. 6a,b). In contrast to TAMs in glioblastoma $^{19,21}$, we did not observe a loss of M2-polarization of TAMs in response to CSF1R inhibition. To further interrogate the consequences of CSF1R inhibition on TAM activation states, we performed gene set ORA to identify pathways that are enriched in BLZ945-treated TAM-MG and TAM-MDM compared to the vehicle-treated TAM populations, when contrasted to their normal counterparts isolated from tumor-free mice (Extended data Fig. 6f). BLZ945treated TAMs showed an enrichment of gene sets that are associated with neuro-inflammation typically found in neurological disorders including EAE/MS or Alzheimer's disease, such as TNF and IL6 (Extended data Fig. 6f) ${ }^{36,37}$. Gene signatures associated with metabolic modification and cellular responses that indicate the production of nitric oxide (NO) and reactive oxygen species (ROS), were also elevated (Extended data Fig. 6f). Collectively, these gene ontology analyses revealed the presence of a signaling network, which includes inducerand effector molecules of neuro-inflammation.

We then investigated putative signaling pathways mediating this phenotype upon CSF1R inhibition by directly comparing the expression of the subset of TAMs that are protected from BLZ945-induced cell death ( $35 \%$ of cells, Extended data Fig. $4 a, b)$ to their vehicle-treated counterparts (Fig. 6c). Our data indicate a shift from CSF1R to CSF2Rb-STAT5 downstream signaling, as an unbiased ORA analysis of the DEGs revealed an overrepresentation of CSF2Rb- and STAT5-associated pathways in BLZ945-treated MG (Fig. 6c). The induction of a pathway associated with production of MMP14 (Fig. 6c), a protease that has been correlated with demyelination in neuro-inflammation ${ }^{38}$, was also observed. Moreover, our data further point towards the induction of IL4-driven pathways (Fig. 6c, see also Extended data Fig. 6f) that were associated with wound healing processes in recurrent glioblastoma in the context of long-term CSF1R inhibition ${ }^{20}$. Activation of CSF2R downstream signaling was further confirmed by elevated expression of known CSF2R downstream genes and transcription factor activation (Fig. 6d,e). 
To further validate that CSF1R blockade leads to induction of a compensatory CSF2RbSTAT5 signaling axis, we compared the gene expression in TAMs from vehicle and BLZ945treated animals to published signatures of CSF1 and CSF2 downstream signaling in MG ${ }^{39}$. GSEA confirmed that in particular 7d-BLZ945-treated TAM-MG in BrM express signatures of CSF2 activation while vehicle-treated TAM-MG showed signatures associated with CSF1 activation (Fig. 6f). Gene signatures indicative of compensatory CSF2Rb signaling remained evident in samples isolated from mice that were treated with BLZ945 longer than the 7d perioddenoted as BLZ endpoint (EP) (Fig. 6g). In contrast to TAM-MG, TAM-MDM lacked the CSF2 gene signature enrichment in response to CSF1R inhibition in vivo (Fig. 5f), although both cell types respond to CSF2 in vitro (Extended data Fig. 7a,b), express Csf2ra and Csf2rb (Extended data Fig. 7c), and show up-regulation of putative CSF2 target genes and STAT5 transcription factor activity in response to CSF1R inhibition (Fig. 6d,e). Taken together, our data indicate that CSF1R inhibition leads to the induction of a compensatory CSF2Rb-STAT5 signaling axis that protects a subset of TAMs from BLZ945-induced cell death in BrM. However, compensatory CSF2Rb-STAT5 activation induces TAM gene signatures that have been associated with neuroinflammation and which trigger IL4-mediated wound repair mechanisms (summarized in Extended data Fig. 7d).

\section{Tumor-associated niche cells are the primary source of CSF2 in BrM}

We next sought to identify the cellular source of CSF2 in BrM. Gene expression analyses indicated that TAM-MG and TAM-MDM express no or low Csf2 levels respectively, with no change in expression levels in response to CSF1R inhibition (Extended data Fig. 7c). FACSsorted MDA-BrM tumor cells showed low CSF2 mRNA expression, however, we did not detect CSF2 protein in tumor cells in MDA-BrM and 99LN-BrM lesions by IF staining (Fig. 7a). Histological assessment revealed that the dominant cellular source for CSF2 in vehicle and BLZ945-treated mice are vessel-associated cells within or in close vicinity to BrM lesions (Fig. $7 a)$, indicating the generation of a protective environment within the vascular niche. Further characterization of the CSF2 expressing cell type revealed that PDGFRb+ pericytes represent the major cellular source for CSF2 in BrM (Fig. 7b). Critically, the TAMs that were still present after 7 days of BLZ945 treatment were localized in close proximity to vessels. By contrast, the number of TAMs localized distal to vessels was greatly reduced in response to CSF1R inhibition (Fig. 7c-d). Consequently, the presence and spatial distribution of CSF2-producing cells relative to TAM-MG and TAM-MDM may determine regional differences in the extent of TAM depletion vs. CSF2-mediated activation in BrM.

Combined targeting of CSF1R and CSF2Rb-STAT5-mediated inflammation leads to more sustained anti-tumor responses in BrM 
We next examined whether combined inhibition of CSF1R and CSF2Rb-STAT5 downstream signaling could prevent the regrowth of BrM after the initial response to BLZ945 treatment. We tested the efficacy of a neutralizing CSF2Rb antibody and the previously described STAT5 inhibitor AC4-130 ${ }^{40}$ to block protective effects of CSF2 in vitro (Extended data Fig.8a). We did not observe any effects on the viability of bone-marrow derived macrophages (BMDM) and EOC2 MG in response to CSF2Rb neutralization using antibody concentrations of $0.05-0.5$ $\mu \mathrm{g} / \mathrm{ml}$ (Extended data Fig. 8b). In contrast, we found that $5 \mu \mathrm{M}$ and $10 \mu \mathrm{M}$ AC4-130 blocked the proliferative and protective capacity of CSF2, while lower concentrations did not affect MG and BMDM viability in vitro (Extended data Fig. $8 \mathrm{c}$ ). This was further confirmed by the dosedependent reduction of STAT5 phosphorylation in the presence of CSF2 following treatment

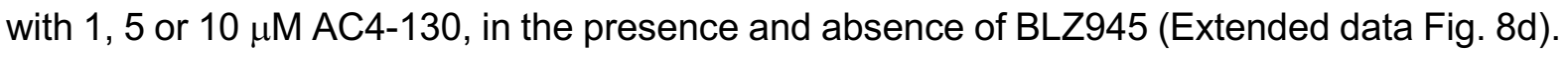
Therefore, we chose the STAT5 inhibitor AC4-130 for further in vivo analysis to investigate the role of compensatory CSF2Rb-STAT5 signaling in response to CSF1R inhibition.

Interestingly, the combination of AC4-130 and BLZ945 in vivo led to significant synergistic anti-tumor effects with reduced tumor growth kinetics until the trial endpoint (Fig. 8a-c). While we observed an initial reduction of the growth rate in AC4-130 or BLZ945 single-treated mice compared to the vehicle treatment group, monotherapy with either inhibitor was ineffective in controlling tumor outgrowth at subsequent time points (Fig. 8b,c). We observed a synergistic effect of the AC4-130 and BLZ945 combination at both d4 and d7 in MDA-BrM mice (Extended data Fig. 8e). However, symptom development resulting from extracranial lesions in this model prevented us from performing longer trials. Histological assessment revealed that BLZ945 treatment in combination with AC4-130 did not lead to more pronounced depletion of the TAM population. Instead we observed a striking change in TAM morphology (Fig. 8d, see also Extended data Fig. 8f). TAMs in the combination treatment group resembled features of ramified microglia with loss of the amoeboid morphology of activated TAMs at the tumorstroma interface or even within the tumor area (Fig. 8d, see also Extended data Fig. 8f). Examination of $M G$ in the adjacent brain parenchyma also indicated morphological changes. BLZ945 treatment led to retraction of protrusions and enlarged cell bodies in the remaining MG population, while combination treatment normalized this phenotype (Fig. 8d, see also Extended data Fig. 8f). AC4-130 monotherapy did not induce pronounced changes in TAM morphology or numbers.

We next analyzed effects on the integrity of the myelin sheath and axonal morphology by transmission electron microscopy as a mean to evaluate tissue damage following the different therapeutic regimens investigated herein, given the indications of neuro-inflammation and neuronal damage. Axons in vehicle-treated animals showed a circular morphology in the peritumor region similar to those found in the adjacent parenchyma. Interestingly, in BLZ945- 
treated BrM we observed irregularly shaped axons with dense myelin. In contrast, axons in BLZ945+AC4-130 treated BrM showed a regular morphology, indicating a reversion of the phenotype observed in response to BLZ945 monotherapy (Fig. 8e,f). We did not observe differences in other characteristics of axonal damage such as inclusions, protrusions or degradation of the myelin sheath as previously described for CSF1R haploinsufficiency ${ }^{41}$. Axons in the normal adjacent brain parenchyma also showed similar morphology across all conditions (Fig. 8e,f). Collectively, our data indicate that combined targeting of CSF1R and STAT5 results in sustained tumor control, concomitant with a phenotypic normalization of the TAM and microglia population as well as reversion of the observed axonal morphology.

\section{DISCUSSION}

Given the critical roles of various tumor-associated immune and stromal cells, TME-targeted therapies are emerging as promising intervention strategies either in combination with standard therapy or as monotherapy ${ }^{42-45}$. To date, a major focus has been directed towards developing $T$ cell-based immunotherapies such as immune checkpoint blockade or CAR T cell therapies ${ }^{46-48}$. However, many cancer types are characterized by low T cell content ${ }^{44,49}$, or the majority of $\mathrm{T}$ cells present in tumors are incapable of executing cytotoxic $\mathrm{T}$ cell responses ${ }^{50}$. T cell-excluded tumors are often highly infiltrated by myeloid cells, which are associated with the establishment of a cancer-permissive, immune-suppressive environment 51. TAMs are one of the most abundant non-cancerous cell types in primary and metastatic brain tumors ${ }^{14}$ and hence represent a promising target for TME-directed therapies ${ }^{16,19,20,52}$. Here we demonstrate that TAM targeting with the CSF1R inhibitor BLZ945 delayed brain metastatic onset and led to temporary stasis of established metastases.

In contrast to our previous findings from glioblastoma mouse models where TAMs survived CSF1R inhibition and were instead re-educated in that context, in BrM we observed depletion of the majority of TAMs, with only a subset being protected from BLZ945-induced cell death. CSF1R inhibition triggered pro-inflammatory responses associated with the production of proinflammatory cytokines as well as ROS and NO. Induction of pro-inflammatory responses has been shown to be beneficial in tumor control of extracranial cancers and displays synergy with chemotherapy in a breast cancer model ${ }^{53}$. By contrast, strategies that potentially initiate proinflammatory responses in the brain have to be very carefully weighed for their ability to achieve efficient tumor control while minimizing the risk of detrimental neurotoxicity. Indeed, our gene expression analysis revealed that the activation status of BrM-TAMs in response to CSF1R blockade resembles features of effector cells that drive demyelination and neurotoxicity in neurodegenerative disorders including EAE/MS and Alzheimer's disease ${ }^{37}$. 
This phenotype can be attributed to the induction of CSF2Rb-STAT5 downstream signaling in BrM-TAMs that were located in close vicinity to CSF2-producing niche cells.

Our data show that CSF1R inhibition in BrM initially induces anti-tumor responses. However, the subsequent activation of a CSF2Rb-STAT5-mediated pro-inflammatory response in the CNS most likely leads to tissue damage followed by wound repair mechanisms to limit neurotoxicity. While we did not find indications for demyelination, we did observe the formation of irregularly shaped axons with dense myelin sheaths following BLZ945 treatment. One potential explanation for this phenomenon could be enhanced re-myelination during neuronal regeneration in combination with reduced de-myelination capacity due to BLZ945-induced MG depletion, thereby leading to altered myelin organization and axonal compression. In addition to a shift from CSF1 to CSF2-mediated TAM activation, our data indicate the induction of IL4mediated responses. This is in line with previous findings in recurrent glioblastoma where an IL4-mediated wound repair signature was associated with tumor recurrence ${ }^{20}$. Importantly, neuronal mimicry by brain metastatic tumor cells as previously reported ${ }^{54-57}$, and as we find in this study in response to CSF1R inhibition, might allow these cells to exploit host responses that aim to limit tissue damage and consequently support tumor outgrowth.

Pharmacological blockade of compensatory CSF2Rb-STAT5 downstream signaling further emphasized the biological consequences of causing pro-inflammatory responses within the CNS. Combined CSF1R and STAT5 inhibition resulted in synergistic anti-tumor effects compared to BLZ945 or AC4-130 monotherapy. In contrast to our initial hypothesis that combined blockade of CSF1R and CSF2Rb-STAT5 signaling would reduce neuroinflammation due to a complete loss of the TAM population, we found a normalization of TAM morphology. This indicates that a combination treatment incorporating BLZ945 and AC4-130 has the potential to revert phenotypes of tumor-mediated TAM alterations with concomitant maintenance of anti-tumor activity. TME-targeted strategies that preserve physiologically essential cell types are of critical clinical significance, in particular in the brain which controls higher cognitive functions and all vital systems. In the future, it will be critical to determine compensatory pathways that remain active upon combined inhibition of CSF1R and STAT5 downstream signaling and to determine the respective TAM activation status in order to evaluate their role in anti-tumor responses.

Taken together, the data presented in this study reveal the risk of unleashing pro-inflammatory responses in BrM upon CSF1R inhibition, but also presents the first experimental evidence for a novel strategy to overcome this adaptive resistance mechanism. By designing rational combination therapies to disrupt tumor-glial communication, we find that this leads to sustained tumor control in conjunction with a normalization of microglia/macrophage phenotypes rather than their depletion, which has evident clinical translational implications. 


\section{Author contributions}

FK, JAJ and LS designed experiments, FK, AS, ASB, TA, MS, KN, RRM, MG, BTE, RLB, PSZ, JZ and LS performed experiments and analyzed data, FK performed computational analysis, MEH, RTD, PNH and KP provided patient samples, FK, JAJ and LS wrote the manuscript. All authors edited or commented on the manuscript. JAJ and LS conceived and supervised the project.

\section{Conflict of interest}

The authors declare no competing interests.

\section{Acknowledgements}

We thank X. Chen, M. Quick, K. Simpson, P. Dinse, A. Möckl, P. Gebhardt and E. De laco for excellent technical support and members of the Joyce lab and the Georg-Speyer-Haus for insightful discussion. We are grateful to Novartis for providing BLZ945, and Dr. Marion Wiesmann, Novartis, for critical input and discussion. We thank the GSH, Goethe University and MSKCC Core Facilities for Molecular Cytology for technical assistance, in particular Marion Basoglu for electron microscopy, and the Core Facility for Microscopy at the IMB Mainz for access to the Imaris software. Research in the lab of JAJ is supported by the Breast Cancer Research Foundation, Ludwig Institute for Cancer Research, University of Lausanne, Swiss Bridge Award, Swiss Cancer League (KFS-3390-08-2016), and Cancer Research UK, as well as research fellowships from the German Research Foundation (KL2491/1-1 to FK and SE2234/1-1 to LS ), Fondation Medic (to FK), and Metastasis Research Center at MSKCC (to LS). Research in the lab of LS is supported by institutional funds from the Georg-Speyer-Haus jointly funded by the German Federal Ministry of Health and the Ministry of Higher Education, Research and the Arts of the State of Hesse (HMWK), as well as grants from the LOEWE Center Frankfurt Cancer Institute (FCl), the German Cancer Consortium (DKTK, partner site Frankfurt/Mainz), the German Cancer Aid (Max-Eder Junior Group Leader Program 70111752), the German Research Foundation (SE2234/3-1), the Beug Foundation for Metastasis Research and the Dr. Bodo Sponholz Foundation. 


\section{References}

1. Ostrom, Q.T., Wright, C.H. \& Barnholtz-Sloan, J.S. Brain metastases: epidemiology. Handb Clin Neurol 149, 27-42 (2018).

2. Stelzer, K.J. Epidemiology and prognosis of brain metastases. Surg Neurol Int 4, S192-202 (2013).

3. Tabouret, E., et al. Recent trends in epidemiology of brain metastases: an overview. Anticancer Res 32, 4655-4662 (2012).

4. Valiente, M., et al. The Evolving Landscape of Brain Metastasis. Trends Cancer 4, 176-196 (2018).

5. Owonikoko, T.K., et al. Current approaches to the treatment of metastatic brain tumours. Nat Rev Clin Oncol 11, 203-222 (2014).

6. Kotecki, N., Lefranc, F., Devriendt, D. \& Awada, A. Therapy of breast cancer brain metastases: challenges, emerging treatments and perspectives. Ther Adv Med Oncol 10, 1758835918780312 (2018).

7. Lim, M., Xia, Y., Bettegowda, C. \& Weller, M. Current state of immunotherapy for glioblastoma. Nat Rev Clin Oncol 15, 422-442 (2018).

8. Caponnetto, S., et al. Cancer immunotherapy in patients with brain metastases. Cancer Immunol Immunother 67, 703-711 (2018).

9. Sevenich, L. Turning "cold" into "hot" tumors - Opportunities and Challenges for RadioImmunotherapy against primary and metastatic brain cancers. Frontiers in Oncology 9(2019).

10. Long, G.V., et al. Combination nivolumab and ipilimumab or nivolumab alone in melanoma brain metastases: a multicentre randomised phase 2 study. Lancet Oncol 19, 672-681 (2018).

11. Tawbi, H.A., et al. Combined Nivolumab and Ipilimumab in Melanoma Metastatic to the Brain. N Engl J Med 379, 722-730 (2018).

12. Aldape, K., et al. Challenges to curing primary brain tumours. Nat Rev Clin Oncol 8, 509-520 (2019).

13. Quail, D.F. \& Joyce, J.A. The Microenvironmental Landscape of Brain Tumors. Cancer Cell 31, 326-341 (2017).

14. Klemm, F., et al. Interrogation of the Microenvironmental Landscape in Brain Tumors Reveals Disease-Specific Alterations of Immune Cells. Cell 181, 1643-1660 e1617 (2020).

15. Friebel, E., et al. Single-Cell Mapping of Human Brain Cancer Reveals Tumor-Specific Instruction of Tissue-Invading Leukocytes. Cell 181, 1626-1642 e1620 (2020). 
16. Sevenich, L., et al. Analysis of tumour- and stroma-supplied proteolytic networks reveals a brain-metastasis-promoting role for cathepsin S. Nat Cell Biol 16, 876-888 (2014).

17. Bowman, R.L., et al. Macrophage Ontogeny Underlies Differences in Tumor-Specific Education in Brain Malignancies. Cell Rep 17, 2445-2459 (2016).

18. Schulz, M., et al. Cellular and Molecular Changes of Brain Metastases-Associated Myeloid Cells during Disease Progression and Therapeutic Response. iScience 23, 101178 (2020).

19. Pyonteck, S.M., et al. CSF-1R inhibition alters macrophage polarization and blocks glioma progression. Nat Med 19, 1264-1272 (2013).

20. Quail, D.F., et al. The tumor microenvironment underlies acquired resistance to CSF$1 \mathrm{R}$ inhibition in gliomas. Science 352, aad3018 (2016).

21. Yan, D., et al. Inhibition of colony stimulating factor-1 receptor abrogates microenvironment-mediated therapeutic resistance in gliomas. Oncogene 36, 60496058 (2017).

22. Qiao, S., Qian, Y., Xu, G., Luo, Q. \& Zhang, Z. Long-term characterization of activated microglia/macrophages facilitating the development of experimental brain metastasis through intravital microscopic imaging. J Neuroinflammation 16, 4 (2019).

23. Andreou, K.E., et al. Anti-inflammatory Microglia/Macrophages As a Potential Therapeutic Target in Brain Metastasis. Front Oncol 7, 251 (2017).

24. Valiente, M., et al. Brain Metastasis Cell Lines Panel: a public resource of organotropic cell lines. Cancer Res (2020).

25. Vareslija, D., et al. Transcriptome Characterization of Matched Primary Breast and Brain Metastatic Tumors to Detect Novel Actionable Targets. J Natl Cancer Inst 111, 388-398 (2019).

26. Doron, H., Pukrop, T. \& Erez, N. A Blazing Landscape: Neuroinflammation Shapes Brain Metastasis. Cancer Res 79, 423-436 (2019).

27. Williams, C.B., Yeh, E.S. \& Soloff, A.C. Tumor-associated macrophages: unwitting accomplices in breast cancer malignancy. NPJ Breast Cancer 2, 15025 (2016).

28. DeNardo, D.G. \& Ruffell, B. Macrophages as regulators of tumour immunity and immunotherapy. Nat Rev Immunol 19, 369-382 (2019).

29. Sevenich, L. Brain-Resident Microglia and Blood-Borne Macrophages Orchestrate Central Nervous System Inflammation in Neurodegenerative Disorders and Brain Cancer. Front Immunol 9, 697 (2018).

30. Nimmerjahn, A., Kirchhoff, F. \& Helmchen, F. Resting microglial cells are highly dynamic surveillants of brain parenchyma in vivo. Science 308, 1314-1318 (2005). 
31. Oishi, K., et al. Identity of neocortical layer 4 neurons is specified through correct positioning into the cortex. Elife 5, e10907 (2016).

32. Bury, F.J., et al. Xenopus BTBD6 and its Drosophila homologue lute are required for neuronal development. Dev Dyn 237, 3352-3360 (2008).

33. Hama, T., et al. Identification and molecular cloning of a novel brain-specific receptor protein that binds to brain injury-derived neurotrophic peptide. Possible role for neuronal survival. J Biol Chem 276, 31929-31935 (2001).

34. Ehrlich, A.T., et al. Expression map of 78 brain-expressed mouse orphan GPCRs provides a translational resource for neuropsychiatric research. Commun Biol 1, 102 (2018).

35. Murray, P.J., et al. Macrophage activation and polarization: nomenclature and experimental guidelines. Immunity 41, 14-20 (2014).

36. Becher, B., Tugues, S. \& Greter, M. GM-CSF: From Growth Factor to Central Mediator of Tissue Inflammation. Immunity 45, 963-973 (2016).

37. Becher, B., Spath, S. \& Goverman, J. Cytokine networks in neuroinflammation. Nat Rev Immunol 17, 49-59 (2017).

38. Mirshafiey, A., Asghari, B., Ghalamfarsa, G., Jadidi-Niaragh, F. \& Azizi, G. The significance of matrix metalloproteinases in the immunopathogenesis and treatment of multiple sclerosis. Sultan Qaboos Univ Med J 14, e13-25 (2014).

39. Re, F., et al. Granulocyte-macrophage colony-stimulating factor induces an expression program in neonatal microglia that primes them for antigen presentation. $\mathrm{J}$ Immunol 169, 2264-2273 (2002).

40. Wingelhofer, B., et al. Pharmacologic inhibition of STAT5 in acute myeloid leukemia. Leukemia 32, 1135-1146 (2018).

41. Chitu, V., et al. Microglial Homeostasis Requires Balanced CSF-1/CSF-2 Receptor Signaling. Cell Rep 30, 3004-3019 e3005 (2020).

42. Brown, J.M., Recht, L. \& Strober, S. The Promise of Targeting Macrophages in Cancer Therapy. Clin Cancer Res 23, 3241-3250 (2017).

43. Marshall, H.T. \& Djamgoz, M.B.A. Immuno-Oncology: Emerging Targets and Combination Therapies. Front Oncol 8, 315 (2018).

44. Peranzoni, E., et al. Macrophages impede CD8 T cells from reaching tumor cells and limit the efficacy of anti-PD-1 treatment. Proc Natl Acad Sci U S A 115, E4041-E4050 (2018).

45. Quail, D.F. \& Joyce, J.A. Microenvironmental regulation of tumor progression and metastasis. Nat Med 19, 1423-1437 (2013).

46. Guedan, S., Ruella, M. \& June, C.H. Emerging Cellular Therapies for Cancer. Annu Rev Immunol 37, 145-171 (2019). 
47. June, C.H., O'Connor, R.S., Kawalekar, O.U., Ghassemi, S. \& Milone, M.C. CAR T cell immunotherapy for human cancer. Science 359, 1361-1365 (2018).

48. Migliorini, D., et al. CAR T-Cell Therapies in Glioblastoma: A First Look. Clin Cancer Res 24, 535-540 (2018).

49. Chongsathidkiet, $\mathrm{P}$., et al. Sequestration of $\mathrm{T}$ cells in bone marrow in the setting of glioblastoma and other intracranial tumors. Nat Med 24, 1459-1468 (2018).

50. Simoni, Y., et al. Bystander CD8(+) T cells are abundant and phenotypically distinct in human tumour infiltrates. Nature 557, 575-579 (2018).

51. Binnewies, M., et al. Understanding the tumor immune microenvironment (TIME) for effective therapy. Nat Med 24, 541-550 (2018).

52. Akkari, L., et al. Dynamic changes in glioma macrophage populations after radiotherapy reveal CSF-1R inhibition as a strategy to overcome resistance. Science Translational Medicine 12 eaaw7843 (2020).

53. Salvagno, C., et al. Therapeutic targeting of macrophages enhances chemotherapy efficacy by unleashing type I interferon response. Nat Cell Biol 21, 511-521 (2019).

54. Zeng, Q., et al. Synaptic proximity enables NMDAR signalling to promote brain metastasis. Nature 573, 526-531 (2019).

55. Wingrove, E., et al. Transcriptomic Hallmarks of Tumor Plasticity and Stromal Interactions in Brain Metastasis. Cell Rep 27, 1277-1292 e1277 (2019).

56. Neman, J., et al. Human breast cancer metastases to the brain display GABAergic properties in the neural niche. Proc Natl Acad Sci U S A 111, 984-989 (2014).

57. Schnepp, P.M., et al. GAD1 Upregulation Programs Aggressive Features of Cancer Cell Metabolism in the Brain Metastatic Microenvironment. Cancer Res 77, 2844-2856 (2017).

58. Jung, S., et al. Analysis of fractalkine receptor CX(3)CR1 function by targeted deletion and green fluorescent protein reporter gene insertion. Mol Cell Biol 20, 4106-4114 (2000).

59. Bos, P.D., et al. Genes that mediate breast cancer metastasis to the brain. Nature 459, 1005-1009 (2009).

60. Ponomarev, V., et al. A novel triple-modality reporter gene for whole-body fluorescent, bioluminescent, and nuclear noninvasive imaging. Eur J Nucl Med Mol Imaging 31, 740-751 (2004).

61. Shree, T., et al. Macrophages and cathepsin proteases blunt chemotherapeutic response in breast cancer. Genes Dev 25, 2465-2479 (2011).

62. Harter, P.N., et al. Distribution and prognostic relevance of tumor-infiltrating lymphocytes (TILs) and PD-1/PD-L1 immune checkpoints in human brain metastases. Oncotarget 6, 40836-40849 (2015). 
63. Yushkevich, P.A., et al. User-guided 3D active contour segmentation of anatomical structures: significantly improved efficiency and reliability. Neuroimage 31, 1116-1128 (2006).

64. Valiente, M., et al. Serpins promote cancer cell survival and vascular co-option in brain metastasis. Cell 156, 1002-1016 (2014).

65. R-core-Team. R: A language and environment for statistical computing. R Foundation for Statistical Computing, Vienna, Austria. https://www.R-project.org/. (2018).

66. Dobin, A., et al. STAR: ultrafast universal RNA-seq aligner. Bioinformatics 29, 15-21 (2013).

67. Love, M.I., Huber, W. \& Anders, S. Moderated estimation of fold change and dispersion for RNA-seq data with DESeq2. Genome Biol 15, 550 (2014).

68. Subramanian, A., et al. Gene set enrichment analysis: a knowledge-based approach for interpreting genome-wide expression profiles. Proc Natl Acad Sci U S A 102 , 15545-15550 (2005).

69. Yu, G., Wang, L.G., Han, Y. \& He, Q.Y. clusterProfiler: an R package for comparing biological themes among gene clusters. OMICS 16, 284-287 (2012).

70. Sergushichev, A.A. An algorithm for fast preranked gene set enrichment analysis using cumulative statistic calculation. bioRxiv (2016).

71. Hanzelmann, S., Castelo, R. \& Guinney, J. GSVA: gene set variation analysis for microarray and RNA-seq data. BMC Bioinformatics 14, 7 (2013).

72. Wickham, H. ggplot2: Elegant Graphics for Data Analysis. . Springer-Verlag New York (2016). 


\section{EXPERIMENTAL MODELS AND SUBJECT DETAILS}

\section{Mice}

All animal studies were approved by the government committee (Regierungspräsidium Darmstadt, Germany) and conducted in the Georg-Speyer-Haus (GSH) in accordance with the requirements of the German Animal Welfare Act, or approved by the Institutional Animal Care and Use Committee of Memorial Sloan Kettering Cancer Center (MSKCC). The Cx3cr1GFP/wildtype (wt) mouse line was generated as described previously ${ }^{58}$. C57BL6, Cx3cr1GFP/wt mice or Athymic/nude mice were purchased from NCl Frederick or Charles River Laboratories or bred within the GSH or MSKCC animal facilities.

\section{Primary cell cultures}

Bone marrow-derived macrophages (BMDM) were derived from monocytes isolated from bone marrow of 6-8 week-old mice. Bone marrow-derived cells were differentiated into macrophages by culture in Teflon bags for $7 d$ in DMEM containing $10 \%$ fetal bovine serum with $1 \% \mathrm{~L}$-glutamine and $1 \%$ penicillin/streptomycin with the addition of $10 \mathrm{ng} / \mathrm{ml} \mathrm{CSF} 1$. Primary microglia were isolated from 6-8 week-old mice using the adult brain dissociation kit (Miltenyi) combined with $\mathrm{CD} 11 \mathrm{~b}+$ magnetic bead enrichment (Miltenyi) according to the manufacturer's instructions. Primary microglia were used for experiments directly after the isolation without further culture on plastic unless otherwise specified. Media for primary microglia contained 1\% FBS, $1 \%$ L-glutamine and $1 \%$ penicillin/streptomycin and were supplemented with $20 \mathrm{ng} / \mathrm{ml} \mathrm{IL34}$ and $5 \mathrm{ng} / \mathrm{ml}$ TGF $\beta$.

\section{Cell lines}

Brain metastatic (BrM) variants of the human breast cancer cell line MDA-MB-231 (here denoted as MDA-BrM ${ }^{59}$ ), H2030-BrM, PC9-BrM and HCC-1954-BrM were provided by Dr. Joan Massagué, MSKCC, New York, and labeled with a triple-imaging vector (TK-GFP-LuC; $\mathrm{TGL}^{60}$ ). The murine parental $99 \mathrm{LN}$ cell line was derived from a metastatic lymph node of the MMTV-PyMT breast cancer model (C57BL6/J background) and twofold selected in vivo for brain homing capacity as previously described ${ }^{17}$, resulting in the $99 \mathrm{LN}-\mathrm{BrM} 2$ variant used herein (denoted as 99LN-BrM throughout this manuscript). The murine parental TS1 and TS2 cell lines were derived from primary tumors of the MMTV-PyMT breast cancer model (FVB/n background) as previously described ${ }^{61}$. The tumor cell lines MCF7, SKBR3, BT474, T47D were purchased from the ATCC. MDA-BrM, 99LN-BrM, TS1 and TS2 cell lines were maintained in DMEM containing $10 \%$ fetal bovine serum with $1 \%$ L-glutamine and $1 \%$ penicillin/streptomycin. The remaining tumor cell lines were maintained in RPMI medium containing $10 \%$ fetal bovine serum with $1 \%$ L-glutamine and $1 \%$ penicillin/streptomycin. The 
microglia cell line EOC2 and the monocytic cell line THP1 were purchased from the ATCC. EOC2 cells were maintained in DMEM containing $10 \%$ fetal bovine serum with $2 \%$ L-glutamine and $1 \%$ penicillin/streptomycin with the addition of $20 \mathrm{ng} / \mathrm{ml} \mathrm{IL34}$ and $5 \mathrm{ng} / \mathrm{ml} \mathrm{TGF} \beta$. THP1 cells were cultured in RPMI medium containing $10 \%$ fetal bovine serum with $1 \%$ L-glutamine and $1 \%$ penicillin/streptomycin. THP1 monocytes were differentiated into macrophages by $24 \mathrm{~h}$ incubation with $150 \mathrm{nM}$ phorbol 12-myristate 13-acetate (PMA, Sigma, P8139) followed by $24 \mathrm{~h}$ incubation in RPMI medium. Human brain microvascular endothelial cells (HBMEC) and human astrocytes (HA) were purchased from Sciencell. HBMECs were cultured on gelatin-coated cell culture dishes, and HA on poly-L-lysine cell culture dishes in endothelial cell media (ECM, Sciencell) $+10 \%$ FBS supplemented with endothelial cell growth factors (ECGF, Sciencell).

\section{Clinical samples}

All participants included in this study provided written consent. Formalin-fixed and paraffinembedded (FFPE) tissue from archived brain metastases collected between 1999 and 2014 was processed as tissue microarrays (TMAs) and provided by P.N.H. and K.H.P.. Specimens were obtained from the UCT tumor bank (Goethe University, Frankfurt am Main, Germany, member of the German Cancer Consortium (DKTK) and German Cancer Research Center (DKFZ), Heidelberg, Germany). Approval for this study was conferred by the ethics committee UCT Frankfurt / Goethe University Frankfurt am Main, Germany; project numbers GS 4/09; SNO_01-12 and SNO_02-2015. The TMAs included specimens from 165 patients. Histopathological scoring of CSF1R expression was obtained from $n=14 / 28$ melanoma, $n=43 / 62$ NSCLC, $n=9 / 9$ SCLC $n=26 / 33$ breast carcinoma, $n=5 / 5$ renal cell carcinoma. Additionally, the TMA contained $n=11$ carcinoma samples, which were not otherwise specified, and $n=10$ rare tumors that were not included in the analyses performed for this study. Patient tissue for whole section immunofluorescent stainings was provided by M.E.H. and R.T.D.. The collection of tumor tissue samples at the Centre Hospitalier Universitaire Vaudois (CHUV, Lausanne, Switzerland) was approved by the Commission cantonale d'éthique de la recherche sur l'être humain (CER-VD, protocol PB 2017-00240, F25 / 99). Additional information about the clinical samples can be found in Extended data Tables 1,2 and ${ }^{62}$.

\section{Generation of experimental brain metastasis and in vivo BLI and MRI measurements}

The initiation of brain metastasis from the MDA-BrM and 99LN-BrM cell lines has previously been described 16,17. Briefly, for brain metastasis generation in xenografted and immunocompetent mice, $1 \times 10^{4}$ MDA-BrM cells or $1 \times 10^{5}$ 99LN-BrM cells were inoculated into the left cardiac ventricle of 6-8 week-old female Athymic/nude mice or 10-12 week-old C57BL6J mice respectively. For a subset of experiments, Cx3cr1-GFP/wt mice were used for 
microglia labeling instead of C57BL6/J wt mice. Tumor progression was monitored by weekly $\mathrm{BLI}$ or MRI measurements in the MDA-BrM and 99LN-BrM models respectively. For BLI measurement, mice were injected with $100 \mu \mathrm{l}$ of D-luciferin (BioCat) subcutaneously and luminescence intensity was measured using an IVIS spectrum in vivo imaging system (Perkin Elmer) with images taken after $1 \mathrm{sec}$ and 1 min exposure times. MR imaging was performed using a 7 Tesla Small Animal MR Scanner (PharmaScan, Bruker) with a volume coil as transmitter and a head surface coil for signal reception. Mice were injected intraperitoneally (i.p.) with $150 \mu \mathrm{l}$ Gadobutrol (Gadovist, $1 \mathrm{mmol} \mathrm{ml}^{-1}$, Bayer) before the measurement. Data acquisition was performed using the Paravision 6.0.1 software with images being acquired in coronal planes. For T2-weighted images, a localized T2-multislice Turbo rapid acquisition with relaxation enhancement (T2 TurboRARE; TE/TR $=33 \mathrm{~ms} / 2500 \mathrm{~ms}$ ) was used while a T1weighted RARE sequence (T1 RARE; TE/TR $=6.5 \mathrm{~ms} / 1500 \mathrm{~ms}$ ) was applied for obtaining T1weighted images. Volumetric analysis of brain metastases was performed on MR image DICOM files using a segmentation tool in the ITK-Snap software ${ }^{63}$.

\section{BLZ945 preclinical trials}

In vivo blood-brain barrier transmigration assay: For in vivo extravasation experiments, Athymic/nude or Cx3cr1-GFP/wt mice were pre-treated for 7 days with $20 \%$ Captisol (vehicle)

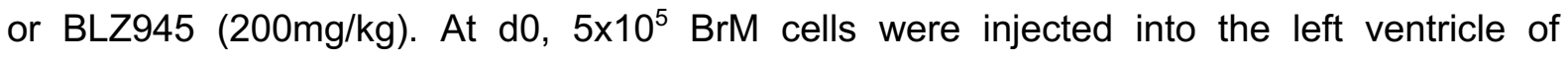
anesthetized mice. MDA-BrM cells were labeled with cell-tracker green (CMFDA) before injection. 99LN-BrM cells were transduced with blue fluorescent protein (BFP) for visualization. For the MDA-BrM model, mice were injected with Texas Red Lectin (Biozol) to visualize the vasculature. Samples were harvested $48 \mathrm{~h}$ after tumor cell inoculation. For the 99LN-BrM model, samples were harvested after 12, 24 and 48h and visualization of the vasculature was achieved by collagen IV staining. Analysis of extravasation was performed on cleared tissue sections as described in the image analysis section.

Prevention trial: For prevention trials, Athymic/nude or C57BL6/J wt mice were treated for 7 days with $20 \%$ Captisol or BLZ945 (200mg/kg). At d0, $1 \times 10^{4}$ MDA-BrM cells or $1 \times 10^{5}$ BrM 99LN-BrM cells were injected into the left ventricle of anesthetized mice. Mice were treated once daily with vehicle or BLZ945 until mice developed symptoms from BrM. Tumor onset and progression was monitored by weekly BLI or MRI measurements.

Intervention trial: For intervention trials, mice were injected with MDA-BrM or 99LN-BrM cells as above. Tumor progression was monitored by weekly BLI or MRI measurements. Once mice showed signs of established BrM defined as a BLI output $>1 \times 10^{6}$ photons $\mathrm{sec}^{-1}$ or MRI volume $>1 \mathrm{~mm}^{3}$, mice were stratified into vehicle or BLZ945 treatment groups at d-1 and treatment was commenced at d0 with daily doses of $20 \%$ Captisol or BLZ945 (200 mg/kg). BrM burden 
was evaluated on $\mathrm{d} 4$ and $\mathrm{d} 7$ after treatment initiation, followed by weekly $\mathrm{BLI}$ or MRI measurements. For the short-term trials, mice were sacrificed on $\mathrm{d} 7$ after treatment initiation. For the survival trials, animals were treated until they developed symptoms from BrM or reached a maximum volume of $>100 \mathrm{~mm}^{3}$ based on MRI measurements or $5 \times 10^{9}$ photons $\mathrm{sec}^{-}$ ${ }^{1}$ based on BLI measurements.

Combination trials: For combination trials, mice were injected with MDA-BrM or 99LN-BrM cells as above. Tumor progression was monitored by weekly BLI and MRI measurements. Once mice showed signs of established BrM, defined as a BLI output $>1 \times 10^{6}$ photons $\mathrm{sec}^{-1}$ or MRI volume $>1 \mathrm{~mm}^{3}$, the animals were stratified into vehicle, BLZ945, AC4-130 ${ }^{40}$, or BLZ945+AC4-130 treatment groups at $d-1$ and treatment was commenced at d0 with daily doses of 20\% Captisol, BLZ945 (200 mg/kg/d), AC4-130 (25 mg/kg/d) or BLZ945+AC4-130. BrM burden was evaluated on $\mathrm{d} 4$ and $\mathrm{d} 7$ after treatment initiation followed by weekly MRI measurements until the trial endpoint.

\section{In vitro blood-brain barrier transmigration assays}

In vitro blood-brain barrier (BBB) transmigration assays were performed as previously described ${ }^{16,59}$. The artificial BBB was formed with HBMECs $(20,000$ cells/well) in co-culture with HA cells $(100,000$ cells/well) on transwell inserts with fluoroblok membranes. For conditions with primary microglia, or the EOC2 microglia cell line, cells were seeded onto the astrocyte cell layer within the last hour of the $5 \mathrm{~h}$-seeding period. After the different cell types were seeded, the artificial BBB was allowed to form and tighten for 3 days. Cell-tracker green (CMFDA)-labeled MDA-BrM and 99LN-BrM cells were allowed to transmigrate for $18 \mathrm{~h}$ through the artificial BBB towards a FBS gradient in the presence or absence of BLZ945 (670 nM). Tumor cell migration through empty inserts (coated with gelatin and poly-L-lysine) with or without the addition of primary BMDMs or microglia was used to determine the effect of BMDMs and microglia on tumor cell migration in the absence of an artificial BBB to determine the baseline ability of those cells to alter the migration of tumor cells. The number of transmigrated tumor cells was quantified by analyzing 25 fields of view (FOV) that were acquired with a 20x objective on a Zeiss Axiolmager Z1 using TissueQuest analysis software (Tissue Gnostics) or CQ1 analysis software (Yokogawa), respectively. Analysis was performed blinded to the group allocation.

\section{Ex vivo brain slice assays}

Organotypic slice cultures were prepared from 6-8 week-old Cx3cr1-GFP/wt mice. Before tissue harvest, mice were treated for 7 days with BLZ945 $(200 \mathrm{mg} / \mathrm{kg})$ to deplete microglia. Vehicle control mice were treated with $20 \%$ Captisol. Fresh brain tissue was collected from 
PBS-perfused mice and cut in $250 \mu \mathrm{m}$ slices using a vibratome (Leica). Brain slices were transferred in transwell inserts in brain slice media as previously described ${ }^{64}$. Brain slices were incubated at $37^{\circ} \mathrm{C}$ and $5 \% \mathrm{CO} 2$ for 2 hours, before $1 \times 10^{4}$ tumor cells (CXMFA+ 99LNBrM) suspended in $1 \mu \mathrm{l}$ media were placed on top of the brain slices. Brain slices were transferred in to the CQ1 (Yokogawa) for time lapse imaging for 18h with images taken every 15 mins at $37^{\circ} \mathrm{C}$ and $5 \% \mathrm{CO} 2$. The number of contacts between tumor cells and microglia were quantified and numbers of tumor cells on brain slices were counted in $25 \mathrm{FOV}$. Analysis was performed blinded to the group allocation.

\section{Proliferation assays}

Cell growth rate was determined using the MTS cell viability assay (Promega). Briefly, cells were plated in triplicate in 96-well plates. $1 \times 10^{3}$ cells for MDA-BrM and 99LN-BrM cells, $5 \times 10^{3}$ cells per well for BMDM and EOC2. For all experiments, media was changed every 48h. Cells

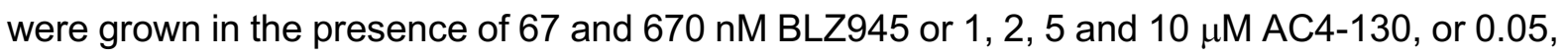
$0.1,0.25$ and $0.5 \mu \mathrm{g} / \mathrm{ml} \mathrm{CSF2R}$ blocking antibody (RnD Biosystems). BMDM and EOC2 were supplemented with recombinant CSF1 or IL34+TGF $\beta$ respectively, unless otherwise indicated. For testing the efficacy of CSF2Rb or STAT5 inhibition, BMDMs or EOC2 were supplemented with CSF2 unless otherwise indicated. For BMDMs and EOC2 stimulated with tumor cellconditioned media (Tu-CM), recombinant growth factors were not added to the media. Reduction of the MTS substrate was detected by colorimetric analysis using a plate reader according to the manufacturer's instructions. $20 \mu$ lof MTS labeling reagent was added to each well. Absorbance was measured after 2 hours at $490 \mathrm{~nm}$ and $650 \mathrm{~nm}$ on a SpectraMax $340 \mathrm{pv}$ plate reader (Molecular Devices).

\section{RNA isolation, cDNA synthesis and quantitative real-time PCR}

RNA was isolated with Trizol, DNase treated, and $1 \mu \mathrm{g}$ of RNA was used for cDNA synthesis. The following Taqman assays were used for qRT-PCR: CSF1R Hs06911250_m1 and Csf1r

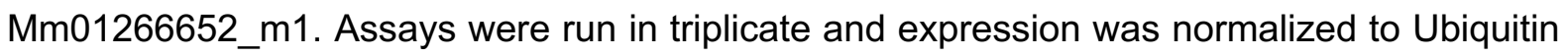
C (UBC Hs00824723_m1 and Ubc Mm02525934_g1) for each sample.

\section{Protein isolation and Western blotting}

For analysis of phosphorylated STAT5 in BMDMs and the MG cell line EOC2, cells were cultured in the absence of growth factors for $12 \mathrm{~h}$. Cells were subsequently stimulated for 5 min with DMEM medium supplemented with CSF1, IL34 or CSF2 (RnD Systems, $10 \mathrm{ng} \mathrm{ml}^{-1}$ ) or Tu-CM. Whole protein lysates were isolated with RIPA buffer containing 1x complete Mini protease inhibitor cocktail (Roche) and 1x PhosphoSTOP phosphatase inhibitors cocktail 
(Roche). STAT5 activation was compared between vehicle and BLZ945-treated (670 nM) BMDMs and EOC2 using antibodies specific to the Tyr694 of STAT5 relative to STAT5 levels. Details about antibodies used for immuno-blotting can be found in the Extended data Table 6.

\section{Tissue preparation and immunostaining}

Tissue for frozen histology was fixed in 4\% PFA overnight and subsequently transferred into $30 \%$ sucrose until the tissue was fully equilibrated. Tissues were then embedded in OCT (Tissue-Tek) and $10 \mu \mathrm{m}$ cryostat tissue sections were used for subsequent analyses. For immunofluorescence staining, frozen sections were thawed and dried at room temperature and rehydrated. For standard staining protocols, tissue sections were blocked in $3 \%$ $\mathrm{BSA}+0.1 \%$ Triton $\mathrm{X} 100$ in PBS for $1 \mathrm{~h}$ at room temperature, followed by incubation with primary antibodies in $1.5 \% \mathrm{BSA}$ overnight at $4^{\circ} \mathrm{C}$. An additional blocking step with the mouse on mouse blocking kit (MOM, Vector Laboratories) was performed for $1 \mathrm{~h}$ at room temperature for primary antibodies derived from mouse. Primary antibody information is listed in the Key Resources Table. Fluorophore-conjugated secondary antibodies were used at a dilution of $1: 500$ in $1.5 \%$ BSA in PBS for $1 \mathrm{~h}$ at room temperature. Consecutive staining protocols were performed if two primary antibodies were derived from the same species.

Paraffin-embedded patient sections were processed using a Leica Bond Max automated staining device. The automated deparaffinization / rehydration, citrate buffer-based antigen retrieval, and blocking of unspecific protein binding and endogenous peroxidase was followed by incubation with primary antibodies (Extended data Table 6), followed by HRP labeled secondary antibodies and DAB conversion.

Patient tissue for immunofluorescence was processed as described previously ${ }^{14}$. Briefly, tissue was OCT embedded by submersion in 2-methyl butane cooled with dry ice, $10 \mu \mathrm{m}$ sections were thawed, air dried and fixed with 100\% methanol. Fixed sections were rehydrated, permeabilized with PBS $+0.2 \%$ Triton $\mathrm{X}-100$ for 3 hours and blocked with PBS $+0.5 \%$ Tween $20+1 \%$ TSA blocking reagent (Perkin Elmer). Primary antibody (Extended data Table 6) incubations where performed overnight in the same buffer, followed by secondary antibody incubations for 1 hour. Directly-conjugated primary antibodies were used after primary and secondary antibody stainings.

For reconstruction of cellular morphology, PFA-fixed brain samples were sliced in $350 \mu \mathrm{m}$ thick sections using a Vibratome VT1200S (Leica). Brain slices were cleared using the XClarity tissue clearing system (Logos Biosystem). Tissue clearing was performed at $0.6 \mathrm{~A}$ for $3 \mathrm{~h}$ using the X-Clarity electrophoretic tissue clearing solution. After tissue clearing, unspecific protein binding was blocked with $3 \%$ BSA in PBS containing $0.1 \%$ Triton-X100 followed by 
incubation with primary antibodies (Extended data Table 6) for $24 \mathrm{~h}$ at room temperature and fluorophore-conjugated secondary antibodies were used at a dilution of 1:500 in 1.5\% BSA in PBS for $12 \mathrm{~h}$ at room temperature

\section{Microscopy and image analysis}

Tissue sections were visualized under a Carl Zeiss Axiolmager Z1 microscope equipped with an ApoTome.2 and a Tissue Gnostic stage to allow for automated image acquisition or the confocal quantitative image cytometer CQ1 (Yokogawa). Quantification of IBA1+ macrophages/microglia, GFAP+ astrocytes and the analysis of proliferation and apoptosis were performed using the TissueQuest analysis software (TissueGnostic) as previously described ${ }^{19}$ or the CQ1 analysis software (Yokogawa). For 3D reconstruction, images were acquired with the CQ1 confocal microscope using the 60x objective. A range of $10 \mu \mathrm{m}$ with 100 Z-stacks was acquired. The semi-automatic surface-rendering module in the Imaris software (Bitplane) was used to create 3D volumetric surface objects. To histologically quantify the percentage of intravascular, extravasating or extravasated tumor cells or the localization of proximal and distal macrophages/microglia, brain sections were stained for collagen IV or CD31 to visualize the vasculature. Analysis was performed blinded to the group allocation. Immunfluorescently stained clinical tissue sections were imaged with an Axio Scan.Z1 slide scanner (Zeiss) equipped with a Colibri 7 LED light source (Zeiss) using a PlanApochromat 20x/0.8 DIC M27 cover slip-corrected objective (Zeiss). Cell type identification and CSF1R expression analysis was performed using the VIS Image Analysis software (Visiopharm) as described previously ${ }^{14}$ and analyzed within the R environment (v3.5) ${ }^{65}$.

\section{Transmission electron microscopy}

Mice were anesthetized with Ketamine/Xylazine and transcardially perfused with PBS and glutaraldehyde in 4\% PFA. Fixed tissue was sectioned in $1 \mathrm{~mm}$ thick slices and regions of interest were chosen based on MRI images for peri-tumor areas or within the M2 cortical region with projections into the corpus callosum in the contralateral hemisphere for tumor-free brain. Brain tissue was washed twice with Na-Cacodylate buffer and post-fixation was achieved with $2 \%$ OsO4 in aqua bidest for $2 \mathrm{~h}$ followed by two washing steps in Na-Cacodylate buffer. Fixed tissue was dehydrated in an ascending alcohol series followed by incubation in propylene oxide and infiltration overnight with a mixture of propylene oxide and araldite. Before embedding, tissue was incubated $2 \mathrm{x} 2 \mathrm{~h}$ in pure araldite. Araldite polymerization was performed at $60^{\circ} \mathrm{C}$ for $48 \mathrm{~h} .1 \mu \mathrm{m}$ thin sections were used to define the region of interest under a light microscope and images were acquired on consecutive $50 \mathrm{~nm}$ thin sections using a CM12 transmission electron microscope (Philips) at 4000x magnification. 5\% uranyl acetate 
and lead citrate were used as contrast enhancers. For the quantification of normal and abnormal axons, axonal phenotypes were categorized as normal, irregular morphology, axons with protrusions or inclusions as well as axons with disaggregated myelin sheaths indicative for degradation. Analysis was performed blinded to the group allocation.

\section{FACS analysis and cell sorting}

For blood analysis, mice were bled either via retro-orbital or submandibular routes under isoflurane anesthesia. For cell sorting, mice were anesthetized with Ketamine/Xylazine, blood collected by cardiac puncture and animals were transcardially perfused with PBS. Brain metastases were macrodissected based on ex vivo BLI signal or MRI images and dissociated using the Brain Tumor Dissociation Kit (Miltenyi) and a single cell suspension was generated using the OctoMACS dissociator. For non-tumor bearing controls, cerebella and olfactory bulb was removed, and the remaining cortex was dissociated using the Mouse Tumor Dissociation Kit. Cell suspensions were filtered through a $40 \mu \mathrm{m}$ mesh filter followed by red blood cell lysis. Normal brain and brain metastases samples were incubated with Myelin Removal Beads (Miltenyi). Cell suspensions were incubated for $15 \mathrm{~min}$ at $4^{\circ} \mathrm{C}$ with $\mathrm{FC}$ block followed by incubation with directly conjugated antibody panels for $15 \mathrm{~min}$ at $4^{\circ} \mathrm{C}$. Cell suspensions were (PBS $+2 \%$ fetal bovine serum) and resuspended in a DAPI solution or stained with efluor as live-dead staining. All flow cytometry analyses were performed on a BD Fortessa device and FACS sorting was performed on an Aria III. Cells were sorted directly in Trizol LS and snap frozen in liquid nitrogen. Antibody information for the flow panels can be found in the Extended data Table 5.

\section{RNA sequencing and gene expression analysis}

RNA was isolated by chloroform extraction and isopropanol precipitation using a glycogen carrier. RNA-sequencing libraries were generated with the SMART-Seq preparation kit (CloneTech) and fragmented with the Nextera XT kit (Illumina). Paired end, 150 base pair, sequencing was performed by Genewiz (New Jersey, USA) on an Illumina HiSeq2500. Reads were adapter trimmed and quality clipped using trim_galore (v0.4.3) in paired mode (https://github.com/FelixKrueger/TrimGalore). Trimmed reads were mapped to a concatenated mouse (mm10) and human genome (hg38) using the STAR aligner (v2.5.2b) ${ }^{66}$ with default parameters. Transcript abundance was quantified using STAR with a GTF file from iGenomes (Illumina). Within each sample only species-specific read counts were retained for further analysis. A count matrix was produced in $\mathrm{R}$ and differential gene expression was assessed with DESeq2 (v1.22) ${ }^{67}$, using a log2 fold change cutoff of $+/-1$ and a false discovery rate of $5 \%$ unless otherwise indicated. Gene set overrepresentation analyses were performed with sets from the Molecular Signatures Database ${ }^{68}$ using the clusterProfiler 
$R$ package (v3.10.1) ${ }^{69}$, while gene set enrichment analyses were done with the fgsea $R$ package (v1.8, https://github.com/ctlab/fgsea) ${ }^{70}$ and single sample gene set enrichment was assessed using the gsva $\mathrm{R}$ package $(\mathrm{v} 1.30)^{71}$. Transcription factor activity was assessed as previously described ${ }^{17}$. Plots were graphed using ggplot2 (v3.2) ${ }^{72}$ and heatmaps drawn with the R package pheatmap (v1.0.12, https://cran.r-project.org/package=pheatmap).

\section{Data and code availability}

All sequencing data has been deposited to the GEO under the accession number GSE133887.

\section{Quantification and statistical analysis}

Summary data are presented as mean \pm standard error of the mean (sem), floating bars with lines indicating min, max and median, or Tukey's box plots using GraphPad Prism software v7 or "ggplot2". Numerical data was analyzed using the statistical tests noted within the corresponding sections of the manuscript. Statistical analyses were performed with GraphPad Prism software $v 7$ and $R$ (version 3.5) performing tests as indicated and were considered statistically significant, with ${ }^{*} P<0.05,{ }^{* *} P<0.01$ and ${ }^{* *} P<0.001$. 
Extended data Table 1 Clinical data of the IHC brain metastasis cohort (TMAs)

\begin{tabular}{|l|l|l|}
\hline Primary tumor & $\begin{array}{l}\text { Sex } \\
\text { m/f }\end{array}$ & $\begin{array}{l}\text { Patient age } \\
\text { (years) }\end{array}$ \\
\hline $\begin{array}{l}\text { Colon cancer } \\
(n=11)\end{array}$ & $7 / 4$ & $\begin{array}{l}56-73 \\
\text { median } 66\end{array}$ \\
\hline $\begin{array}{l}\text { Breast cancer } \\
(n=33)\end{array}$ & $0 / 33$ & $\begin{array}{l}32-76 \\
\text { median } 58\end{array}$ \\
\hline $\begin{array}{l}\text { RCC } \\
(n=18)\end{array}$ & $17 / 1$ & $\begin{array}{l}45-76 \\
\text { median } 65\end{array}$ \\
\hline $\begin{array}{l}\text { NSCLC } \\
(n=62)\end{array}$ & $36 / 26$ & $\begin{array}{l}13-80 \\
\text { median } 61\end{array}$ \\
\hline $\begin{array}{l}\text { SCLC } \\
(n=9)\end{array}$ & $4 / 5$ & $\begin{array}{l}43-72 \\
\text { median } 57\end{array}$ \\
\hline $\begin{array}{l}\text { Melanoma } \\
n=28\end{array}$ & $11 / 17$ & NA \\
\hline
\end{tabular}

Extended data Table 2 Clinical data of the IF brain metastasis cohort (whole tissue sections)

\begin{tabular}{|l|l|l|}
\hline Primary tumor & $\begin{array}{l}\text { Sex } \\
\mathrm{m} / \mathrm{f}\end{array}$ & $\begin{array}{l}\text { Patient age } \\
\text { (years) }\end{array}$ \\
\hline $\begin{array}{l}\text { Breast cancer } \\
(\mathrm{n}=9)\end{array}$ & $0 / 9$ & $\begin{array}{l}40-69 \\
\text { median } 54.5\end{array}$ \\
\hline $\begin{array}{l}\text { NSCLC } \\
(\mathrm{n}=7)\end{array}$ & $3 / 4$ & $\begin{array}{l}54-70 \\
\text { median } 61\end{array}$ \\
\hline
\end{tabular}

Extended data Table 3 DEG_TAM_MDABrM; Excel sheet

Expression fold-changes in MG and MDM.

Extended data Table 4 GO_Overrepresentation_TAM-DEG; Excel sheet

$\mathrm{GO}$ analysis results of clusters from Figure $2 \mathrm{c}$.

Extended data Table 5 DEG_Tumor_MDABrM; Excel sheet

Expression fold-changes in MDA-BrM vehicle-treated vs. 7d BLZ.

Extended data Table 6 Antibodies used for histology, western blotting and flow cytometry

Extended data Movie 1 BrainSlice_MG_vehicle; m4v file

Extended data Movie 2 BrainSlice_MG_BLZ945; m4v file 
a
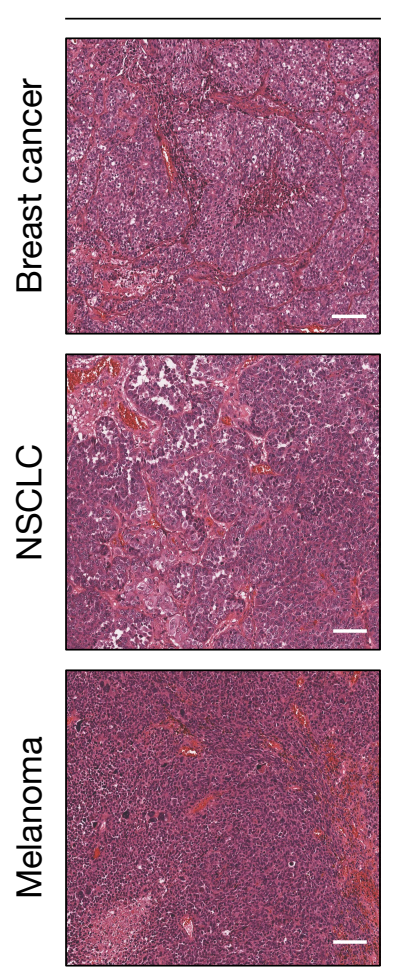

IBA1
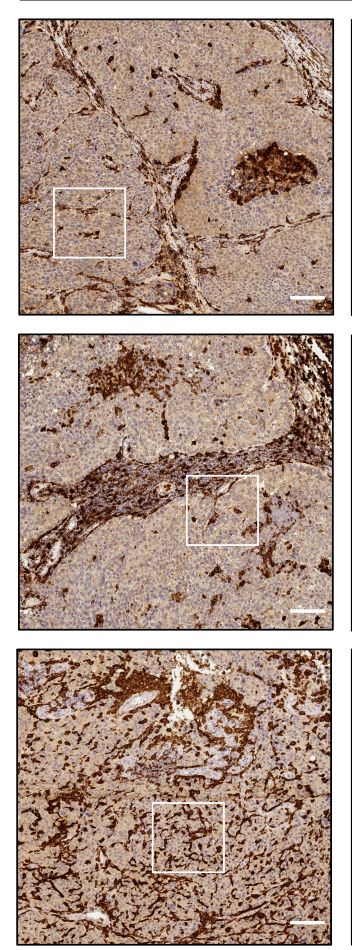
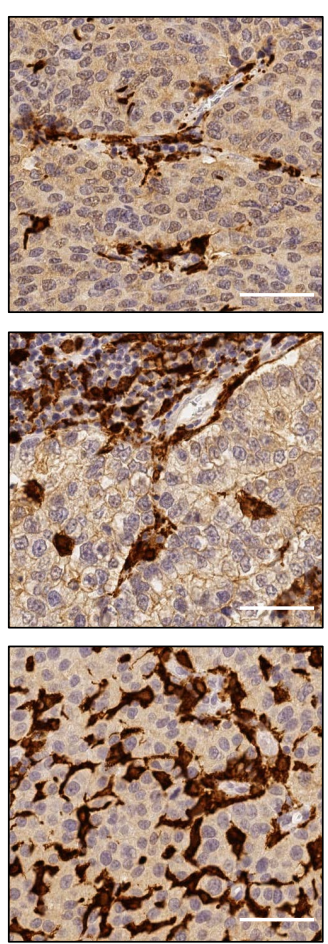

CSF1R
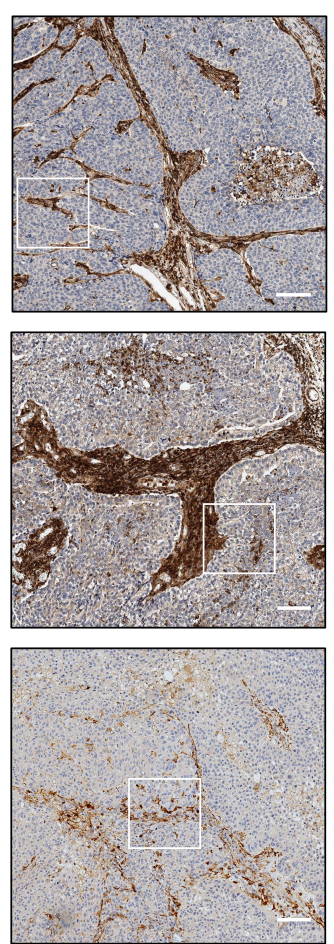
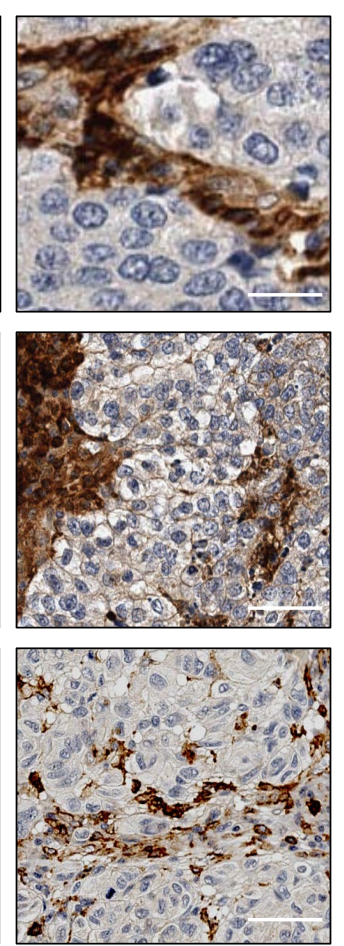

b

Patient BrM

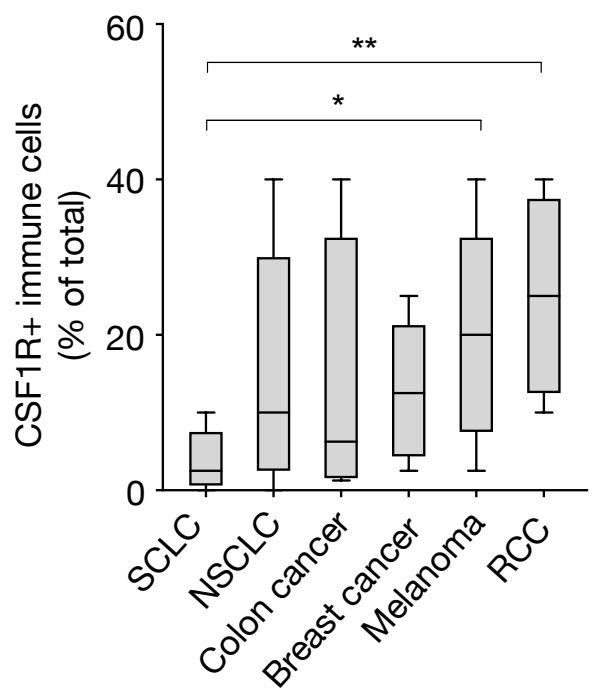

C

Breast-BrM

NSCLC-BrM

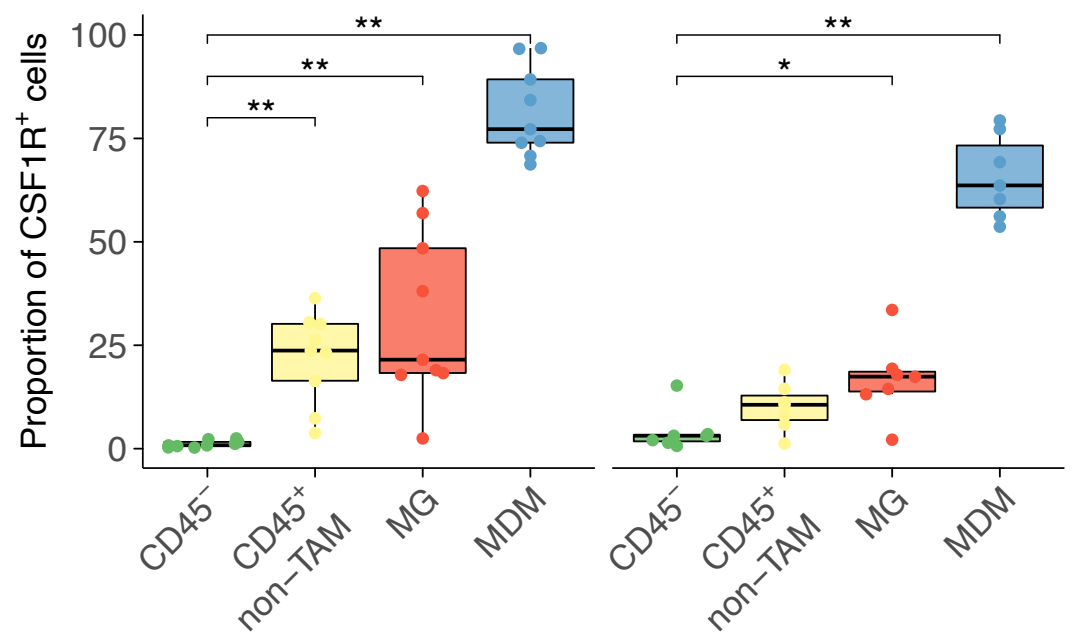


Fig. 1 Tumor-associated microglia and macrophages (TAMs) in human BrM express CSF1R.

a, Representative IHC images of IBA1+ and CSF1R+ cells in human brain metastasis (BrM) samples. Scale bar, 100 and $50 \mu \mathrm{m}$. b, Quantification of CSF1R+ immune cells in BrM originating from NSCLC $(n=43)$, SCLC $(n=9)$, colon $(n=4)$, breast $(n=26)$, melanoma $(n=14)$, and renal cancer $(n=5)$. c, Percentage of CSF1R+ cells in CD45 non-immune cells (CD45), $\mathrm{CD}^{+}$non-TAM immune cells $\left(\mathrm{CD} 45^{+}, \mathrm{CD} 68 / \mathrm{P} 2 \mathrm{RY} 12^{-}, \mathrm{CD} 49 \mathrm{D}^{-1+}\right), \mathrm{MG}\left(\mathrm{CD} 45^{+}\right.$, CD68/P2RY12 $\left.{ }^{+}, \mathrm{CD}^{-} \mathrm{D}^{-}\right)$and MDM $\left(\mathrm{CD}^{+} 5^{+}, \mathrm{CD} 68 / \mathrm{P} 2 \mathrm{RY} 12^{+}, \mathrm{CD} 49 \mathrm{D}^{+}\right)$in BrM originating from breast $(n=9)$ and NSCLC $(n=7)$, as determined by immunofluorescence (IF). Scale bar, $100 \mu \mathrm{m} .{ }^{*} P<0.05,{ }^{* *} P<0.01$; two-tailed Student's t-test in (b), one-sided Wilcoxon signed-rank test in (c). 
a

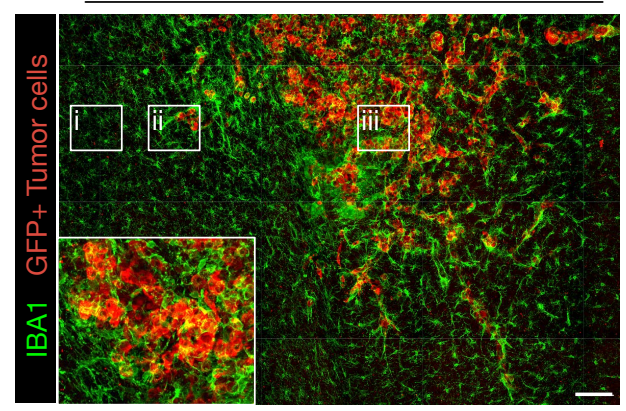

i
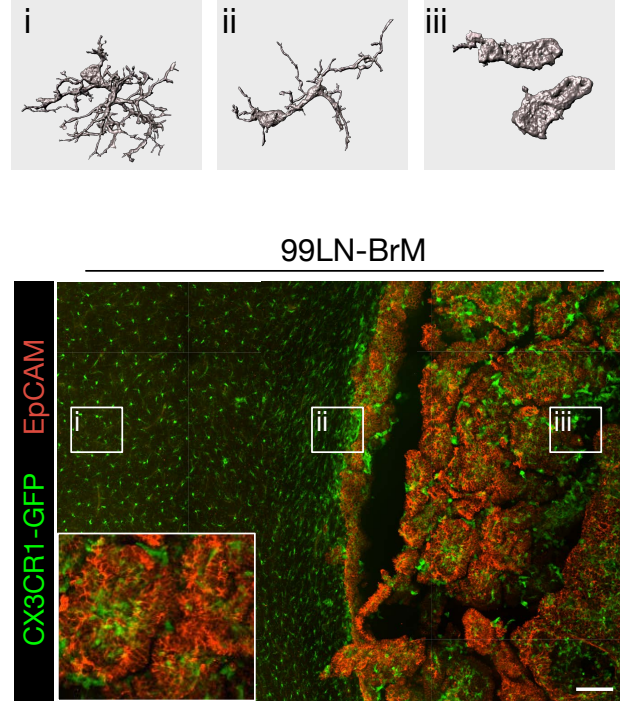

i

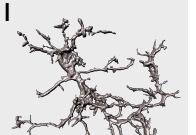

管的

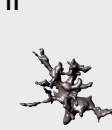

iii

b

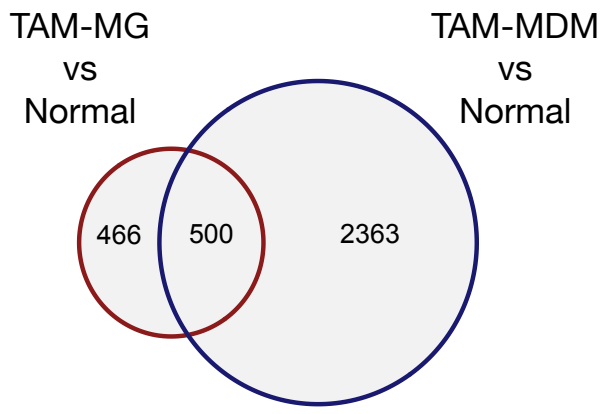

C

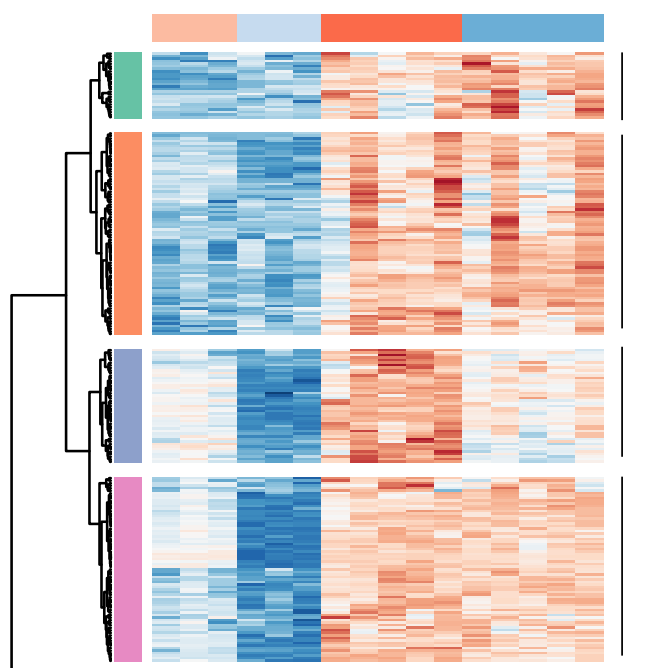

Negative regulation of nervous system development

Lipid localization and transport

Prostaglandin secretion

Metal ion homeostasis

Response to wounding

Hormone secretion and transport

Regulation of neurological system process

Leukocyte mediated immunity

Leukocyte and macrophage migration

Lymphocyte chemotaxis

Negative regulation of immune response

Regulation of vascular smooth muscle proliferation

Regulation of tissue remodeling

Positive regulation of defense response

IFNa response

Cell division

Mitosis

IFNa and IFN $\beta$ response

Antigen processing and presentation via $\mathrm{MHCll}$

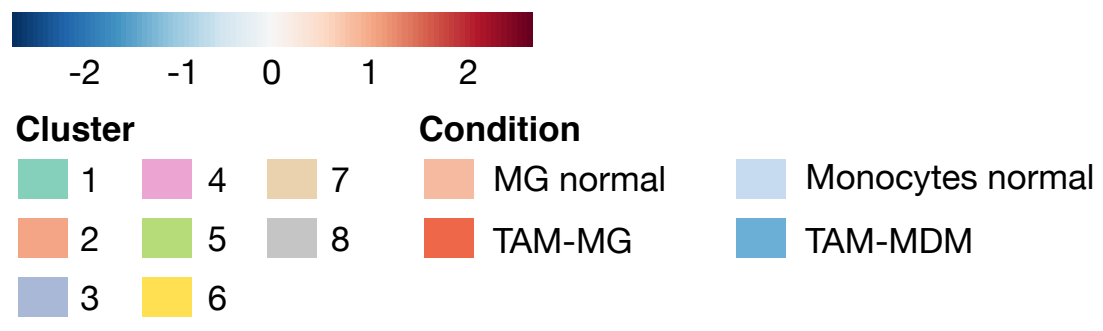


Fig. 2 TAMs exhibit an inflammatory phenotype in mouse BrM.

a, Representative IF images of TAMs in MDA-BrM (upper panel) and 99LN-BrM (lower panel), Scale bar, $250 \mu \mathrm{m}$. 3D image reconstructions of normal microglia (MG) and TAMs are shown in panels (i, ii, iii) below. b, Euler plot of the intersection of MG and monocyte-derived macrophage (MDM) genes upregulated in TAMs in the MDA-BrM model compared to normal controls (i.e. microglia and monocytes respectively from untreated, non-tumor-bearing mice). c, Gene expression heatmap of 500 upregulated genes in TAMs, compared to their normal cellular counterparts. Rows were clustered using Ward's method with 1 - Pearson's correlation coefficient as the distance measurement. Gene ontology terms found overrepresented by gene set overrepresentation analysis (ORA) within each cluster are indicated. 

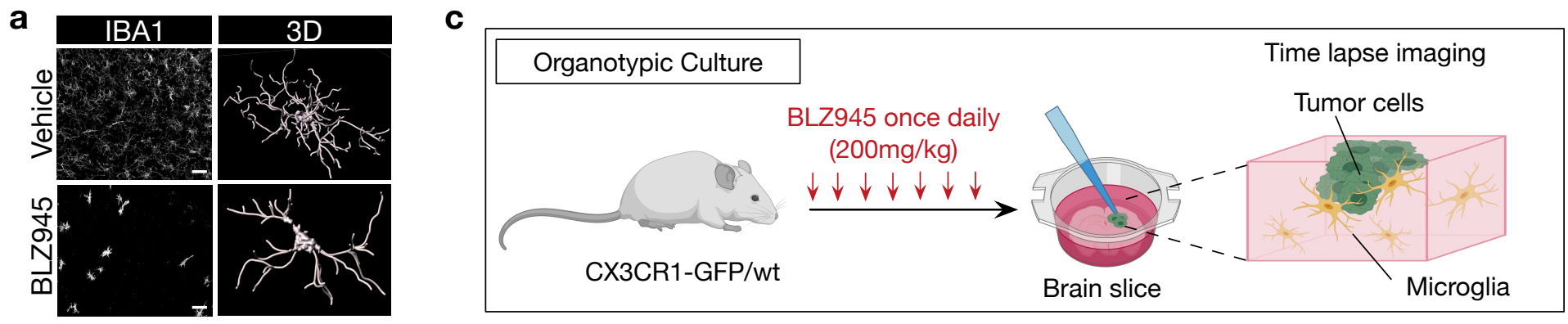

b

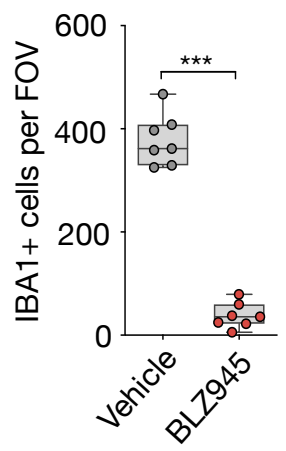

d

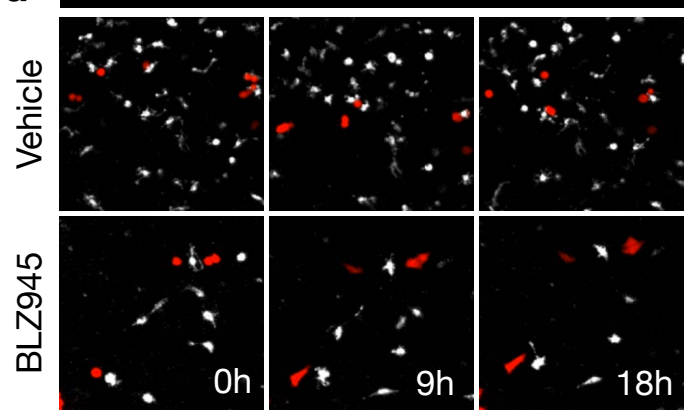

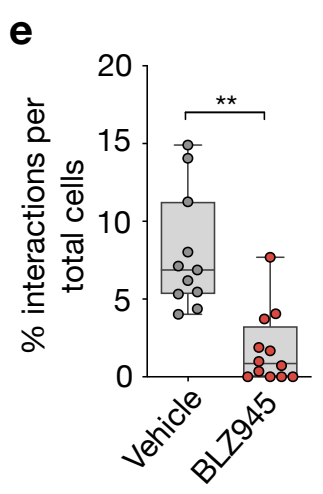

h

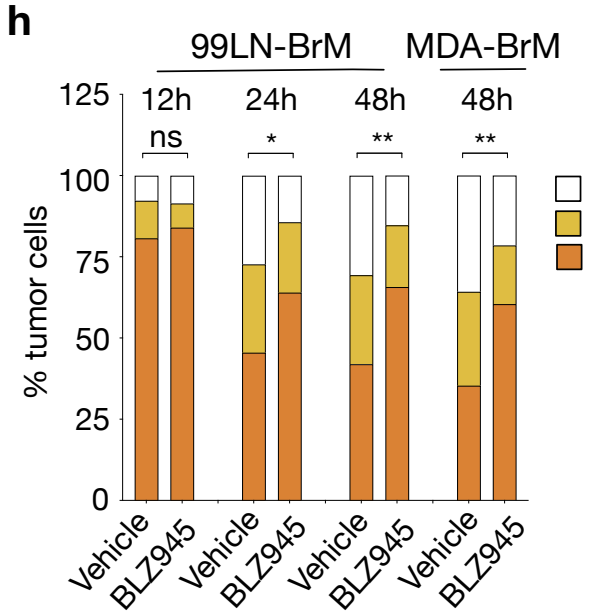

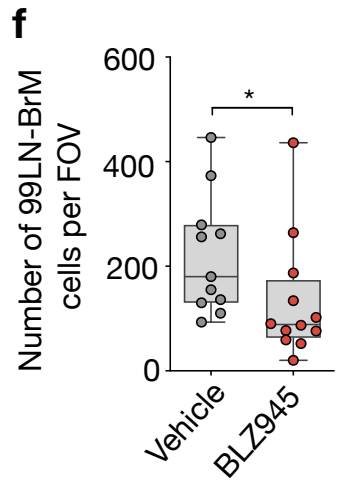

g

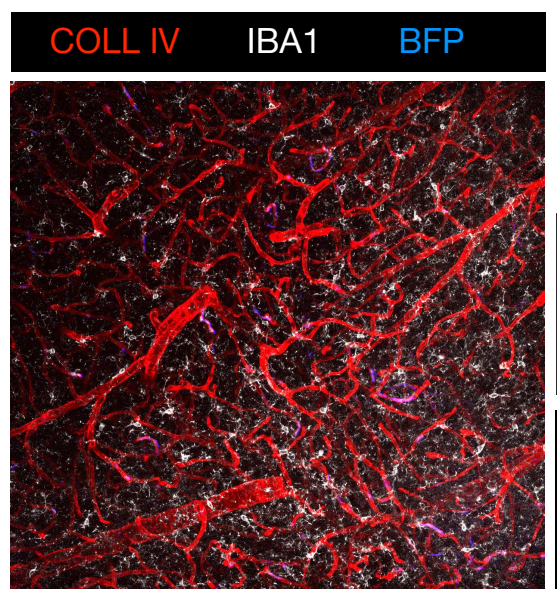

$\mathrm{i}=$ intravascular $\mathrm{i}=$ =extravasating $\mathrm{iii}=$ extravasated
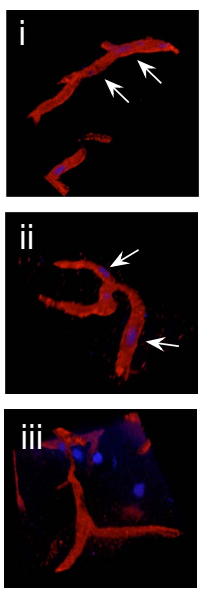

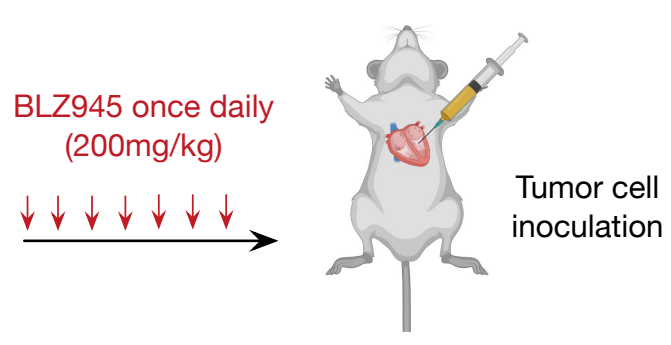

\section{BLZ945 once daily \\ $(200 \mathrm{mg} / \mathrm{kg})$}

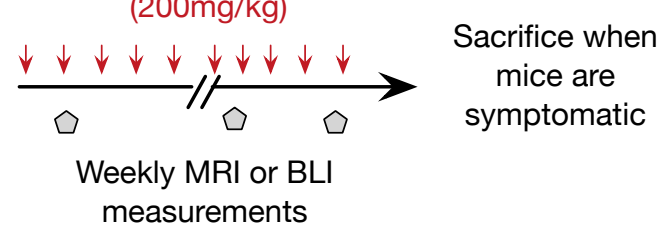

$\mathbf{k}$

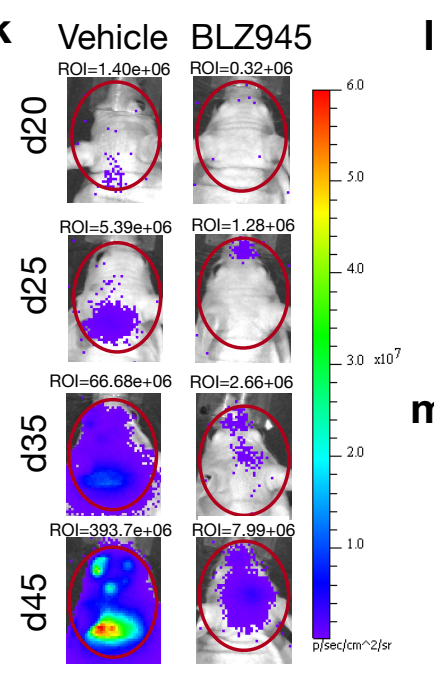

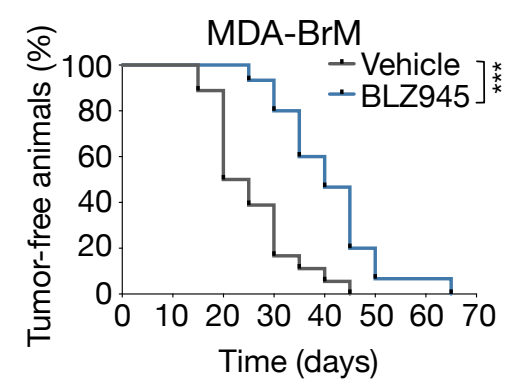

m

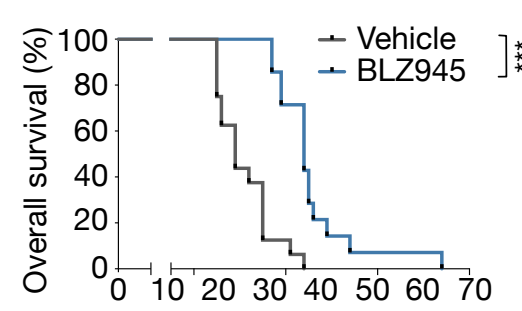

Time (days)
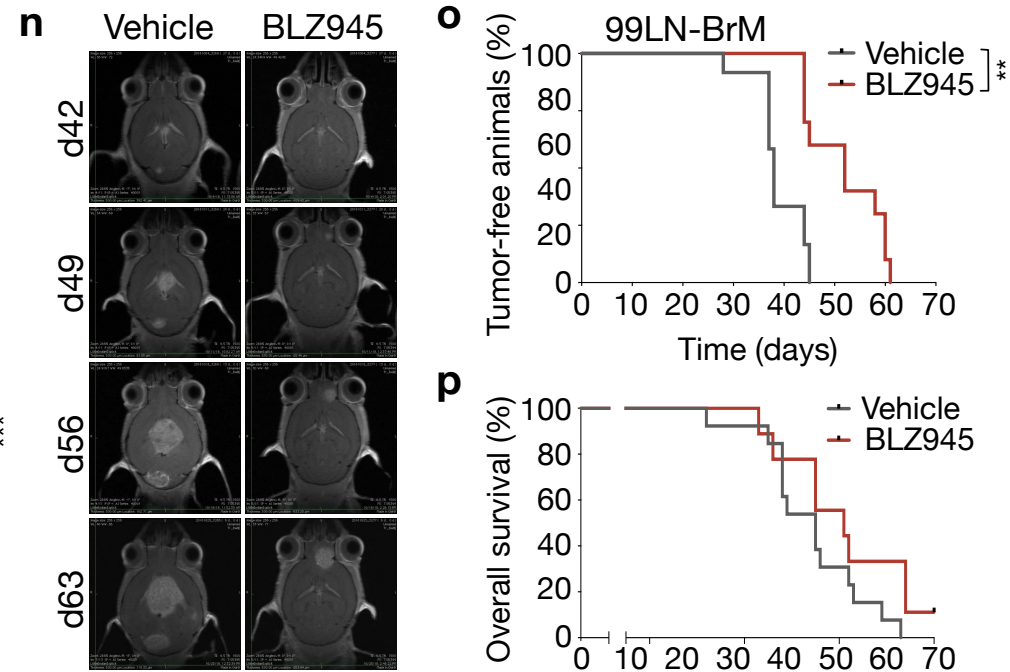

p

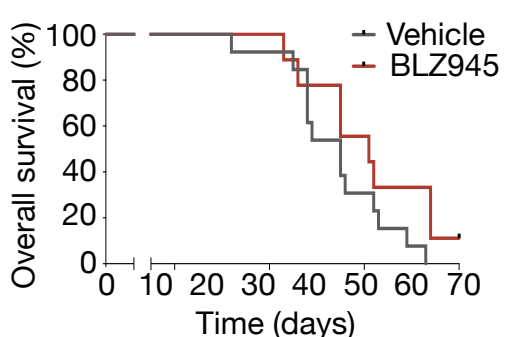


Fig. 3 MG support initial steps of metastatic colonization.

a, Representative IF images (left) and 3D reconstruction (right) of IBA1+ microglia in normal brain parenchyma treated for 7 days with vehicle or BLZ945. Scale bar, $25 \mu \mathrm{m}$. b, Quantification of IBA1 + cells after vehicle or BLZ945 treatment for 7 days, $n=7$ per group with 5 analyzed fields of view (FOV) acquired with a 10x objective. c, Experimental design for timelapse imaging of MG-tumor cell interactions on brain slices isolated from vehicle or BLZ945treated mice. d, Representative images of MG-tumor cell interactions on brain slices at different time points during time-lapse imaging. ( $n=3$ independent experiments). e, Quantification of the number of interactions between tumor cells and MG $(n=11$; vehicle, $n=12$; BLZ945), normalized to the total number of MG in each condition. $\mathbf{f}$, Quantification of tumor cells on brain slices after $20 \mathrm{~h}(\mathrm{n}=11$; vehicle, $n=12$; BLZ945). $\mathbf{g}$, Representative image of a brain section stained for collagen IV (COLL IV, red) and IBA1 (white) to visualize blood vessels and microglia respectively. Insets on right depict the relative localization of tumor cells (BFP, blue) to the vasculature. Arrows indicate intravascular and extravasating cells. $\mathbf{h}$, Quantification of tumor cell extravasation in response to BLZ945 treatment at $12 \mathrm{~h}, 24 \mathrm{~h}$ and 48h after tumor cell injection ( $n=3-5$ mice per condition). i, Quantification of direct contacts between MDA-BrM tumor cells and MG at sites of extravasation ( $n=5$; vehicle, $n=4 ; B L Z 945$ ), normalized to the total number of MG. j, Experimental design of prevention trials. $\mathbf{k}$, Representative BLI images of vehicle and BLZ945-treated MDA-BrM mice at different time points after tumor cell injection. I,m, Kaplan-Meier curves show the percentage of tumor-free animals $(\mathbf{I})$ and the overall survival $(\mathbf{m})$ in the MDA-BrM model $(n=16$; vehicle, $n=14 ; B L Z 945)$. n, Representative MRI images of vehicle and BLZ945-treated 99LN-BrM mice at different time points after tumor cell injection. o,p, Kaplan-Meier curves show the percentage of tumor-free animals (o) and the overall survival $(p)$ in the 99LN-BrM model ( $n=12$; vehicle, $n=10 ; B L Z 945)$. ${ }^{*} P<0.05,{ }^{* *} P<0.01,{ }^{* * *} P<0.001$; two-tailed Student's t-test in (b, e, f, i), Chi square test in (h), Mantel-Cox log rank test in (I, m, o, p). 
a

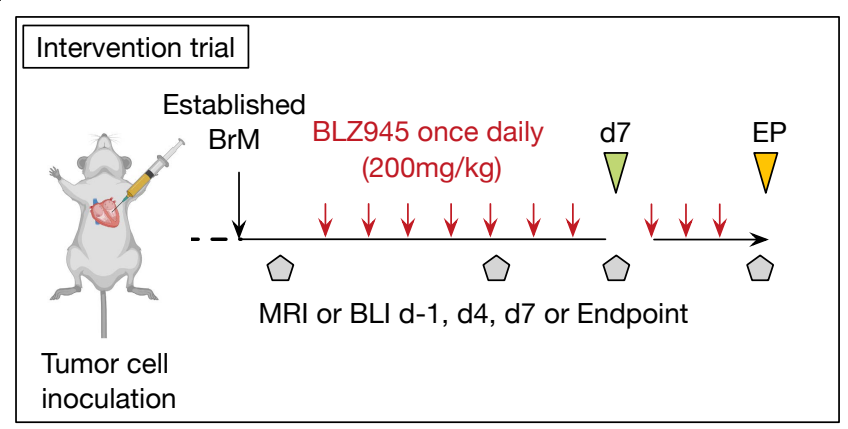

b

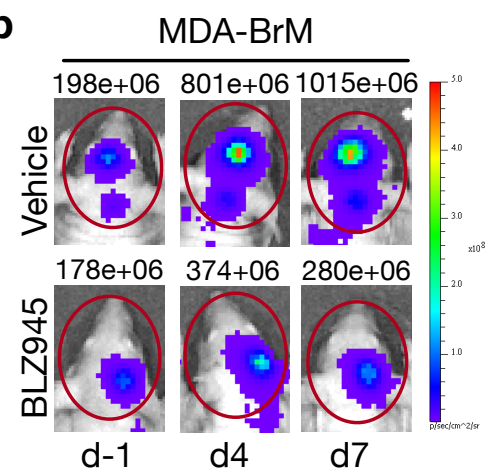

C

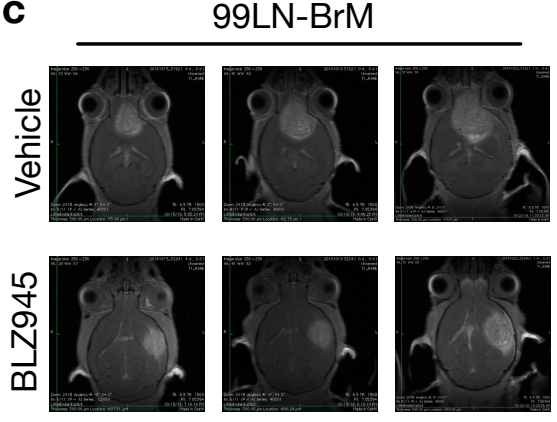

d-1

$\mathrm{d} 7$

f
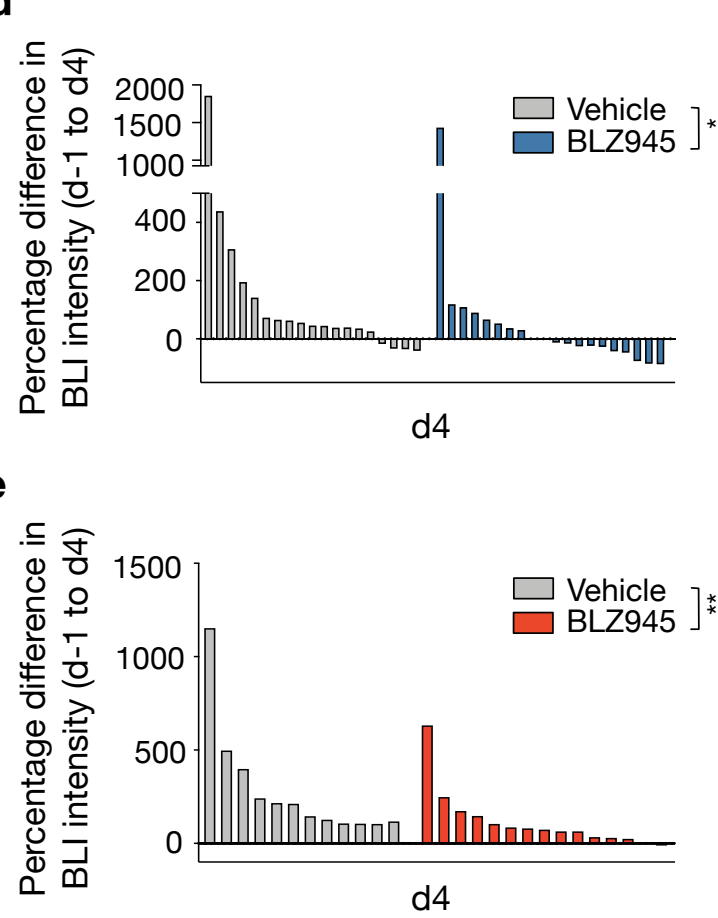

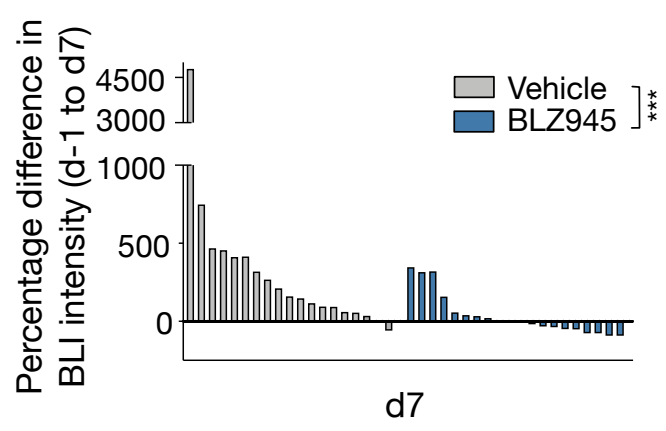

g
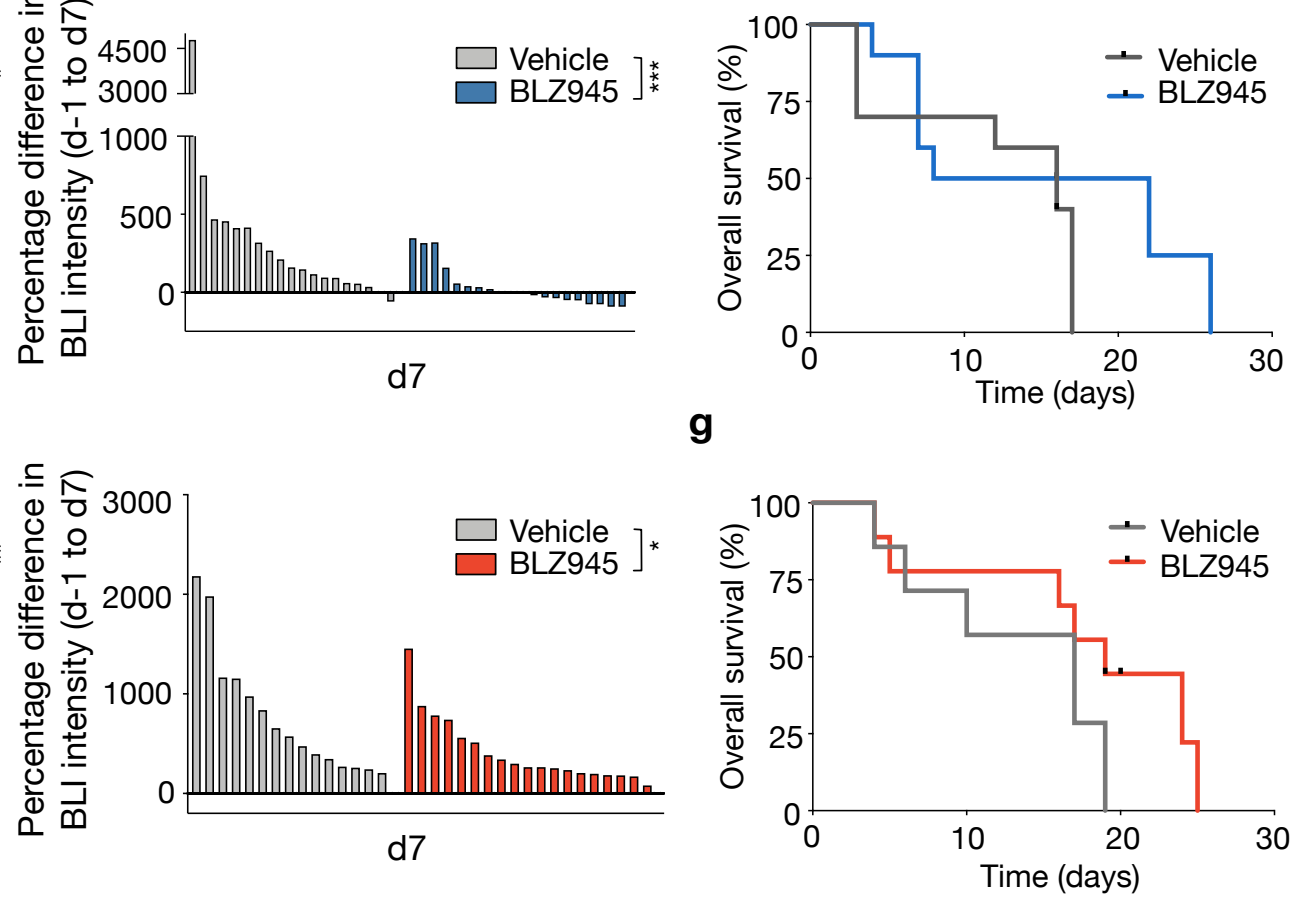
Fig. 4 CSF1R inhibition leads to tumor stasis in short-term trials followed by rapid tumor regrowth.

a, Experimental design of intervention trials. b, Representative BLI images of vehicle and BLZ945-treated MDA-BrM mice. c, Representative MRI images of vehicle and BLZ945-treated 99LN-BrM mice. d,e, Waterfall plots of relative BrM growth at indicated time points (MDA-BrM in (d): $n=19$, vehicle and $n=20, B L Z 945$; and 99LN-BrM in (e): $n=12$ and 15; vehicle and $n=15$ and 19; BLZ945). ${ }^{*} P<0.05,{ }^{* *} P<0.01,{ }^{* * *} P<0.001$, Mann-Whitney U log rank test. f,g, KaplanMeier survival curves of MDA-BrM and 99LN-BrM mice after treatment as indicated in (a) (MDA-BrM in (f): $n=10$, vehicle and BLZ945; and 99LN-BrM in $(\mathbf{g}): n=7$, vehicle and $n=9$, BLZ945, Mantel-Cox log rank test). 
a

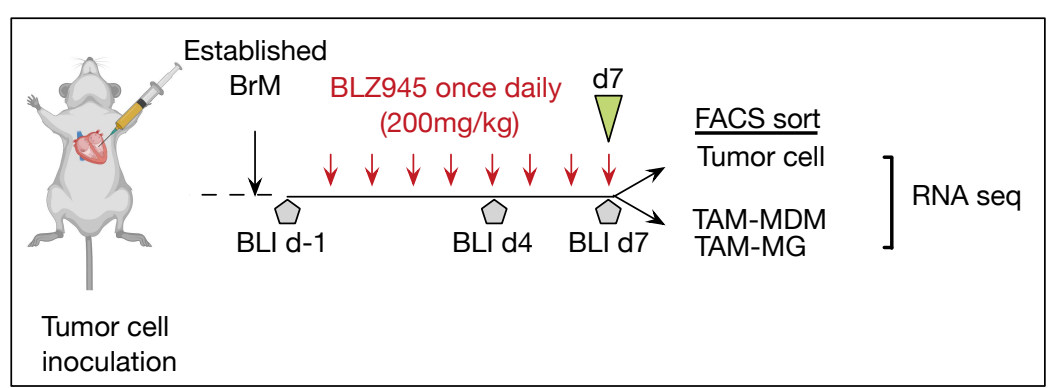

b

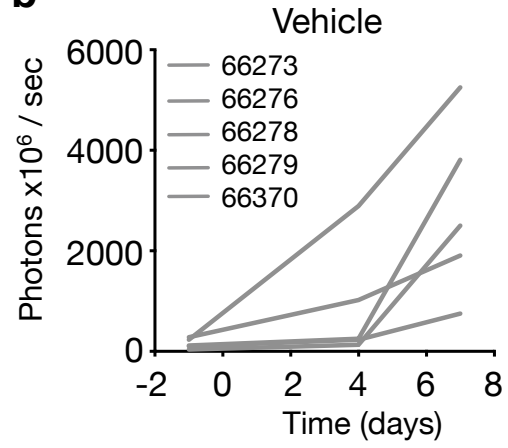

d $\begin{array}{r}\text { Organelle Fission } \\ \text { Chromosome Segregation }\end{array}$ Sister Chromatid Segregation Nuclear Chromosome Segregation Mitotic Nuclear Division DNA Conformation Change Double Strand Break Repair DNA Geometric Change Mitotic Sister Chromatid Segregation DNA Replication DNA Recombination Strand Displacement Negative Regulation Of Cell Cycle Process Chromosome Condensation Negative Regulation
DNA Biosynthetic Process
DNA Repair Protein Localization To Chromosome DNA Integrity Checkpoint

\section{e}

MDA-BrM 7d-BLZ vs. Vehicle treated "Regulation of Neuron Differentiation"

$\mathrm{NES}=1.37, \mathrm{p} \cdot \operatorname{adj}=0.045$

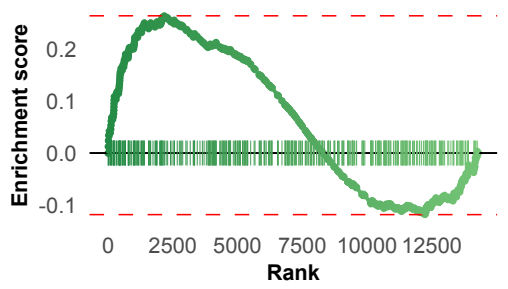

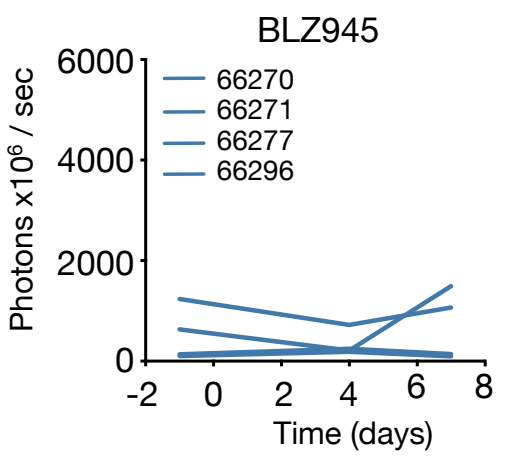

Fraction of gene set

- 0.03

0.06

0.09

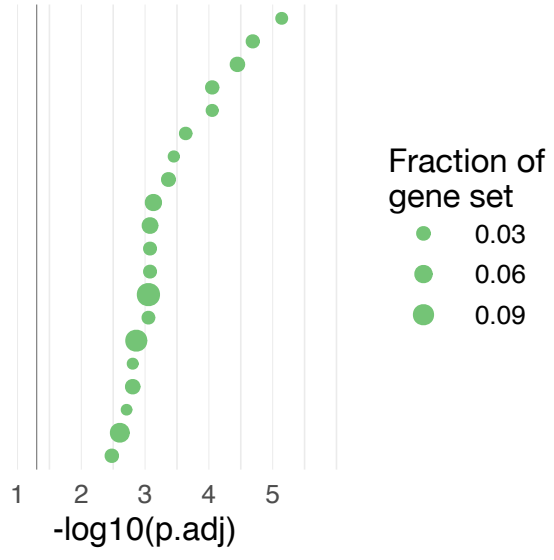

f

MDA-BrM 7d-BLZ vs. Vehicle treated "Cell Morphogenesis involved in Neuron Differentiation" $\mathrm{NES}=1.38$, p.adj $=0.093$

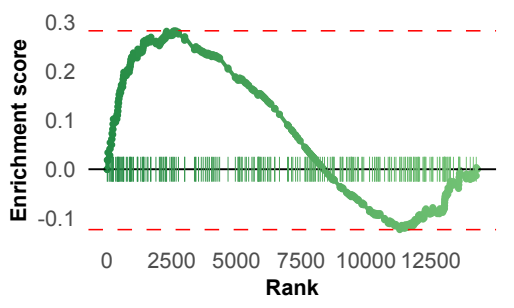

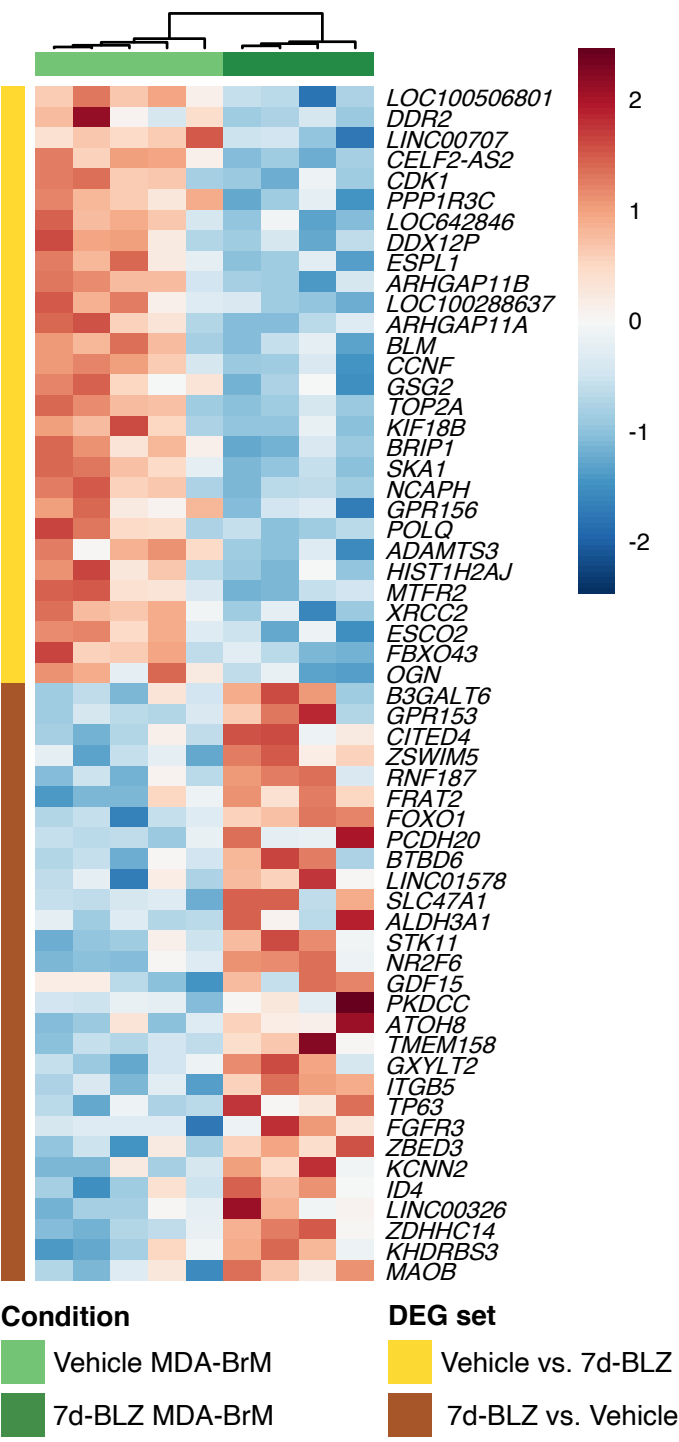
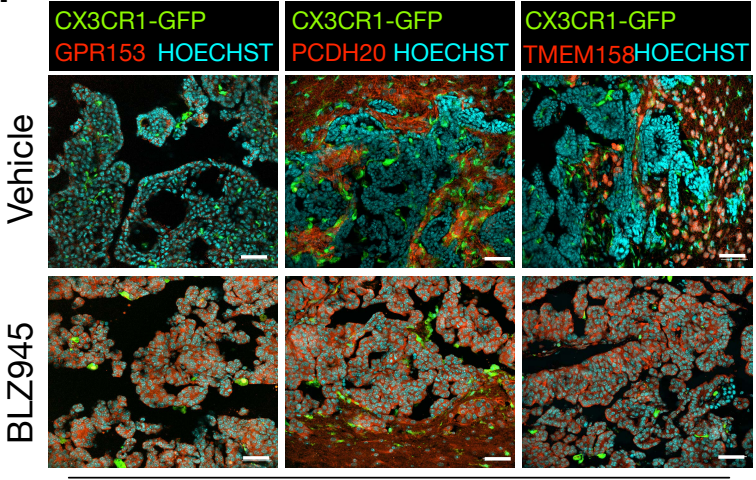

99LN-BrM 
Fig. 5 TAM depletion leads to cell cycle arrest and induction of neuronal signatures in tumor cells.

a, Experimental design for the generation of FACS sorted samples for RNA sequencing. $\mathbf{b}$, Quantification of BLI intensity in vehicle and BLZ945 treated MDA-BrM tumors subjected to RNAseq analysis ( $n=5$; vehicle, $n=4$; BLZ945). c, Heatmap of DEG in MDA-BrM tumor cells in response to 7 days of BLZ945 treatment in vivo $(n=5$; vehicle, $n=4 ; B L Z 945$, p-adjust $\leq 0.1)$. Columns were clustered using Ward's method with 1 - Pearson's correlation coefficient as the distance measurement. Row annotation indicates comparison with differential expression. d, Top 20 (i.e. with lowest p-adjusted) pathways (from the MSigDB GO collection) identified by gene set ORA in the DEG set Vehicle treated vs. 7d-BLZ. e, Gene set enrichment analysis (GSEA) for signatures "Regulation of Neuron Differentiation" and "Cell Morphogenesis involved in Neuron Differentiation" in 7d BLZ945 treated tumor cells compared to vehicle controls. NES, normalized enrichment score. f, Representative images of tumor cellexpressed factors that are associated with neuronal differentiation identified in panel (c). Scale bars, $50 \mu \mathrm{m}$. 


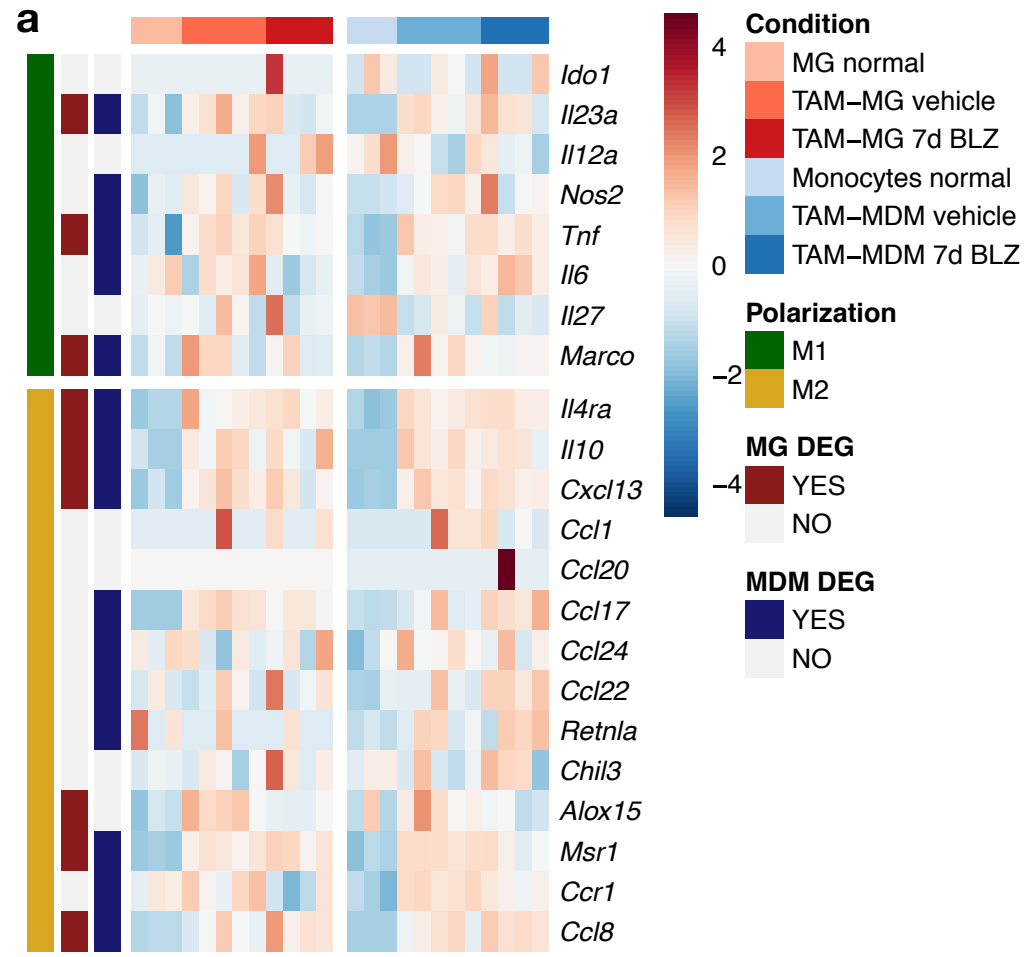

C

Rutella Response To Hgf Vs Csf2rb And II4 Dn Schlosser Serum Response Dn . Rutella Response To Csf2rb And II4 Up . Ross Aml With Pml Rara Fusion . Rutella Response To Hgf Up . Sasson Response To Forskolin Dn . Weng Por Targets Liver Up . Sasson Response To Gonadotrophins Dn Chang :
Core Serum Response Dn Wierenga Stat5a Targets Up . Jiang Tip30 Targets Up Chauhan Response To Methoxyestradiol Up . Maina Vhl Targets Up . Huang Foxa2 Targets Up

Petrova Endothelium Lymphatic Vs Blood Up Zhou Inflammatory Response Fima Dn . Servitja Liver Hnf1a Targets Up 。 Massarweh Tamoxifen Resistance Up . Rozanov Mmp14 Correlated. Nikolsky Breast Cancer 7q21 Q22 Amplicon 。

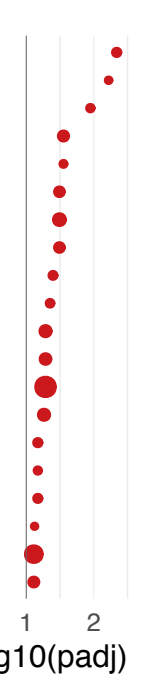

d

Fraction of gene set

Condition

$$
\begin{array}{llll}
-0.0 & 0.1 & \bullet & 0.2
\end{array}
$$

TAM-MG BLZ $00,50,5$

\section{f CSF1 geneset}

7d-BLZ945 vs. Vehicle treated NES $=-1.43$, p.adj $=0.064$

ড

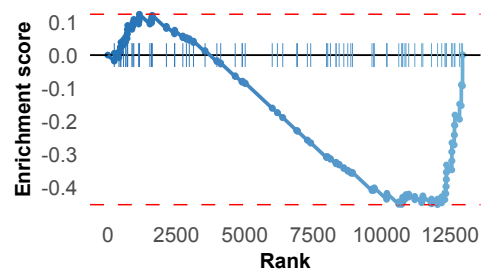

NES $=-1.48, p . \operatorname{adj}=0.018$

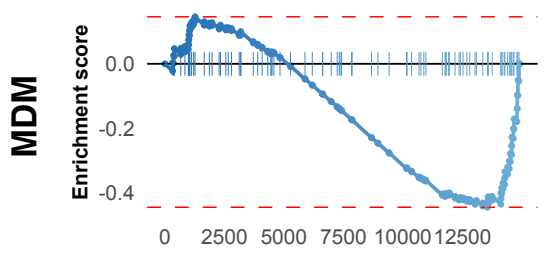

\section{CSF2 geneset}

7d-BLZ945 vs. Vehicle treated

NES $=-1.31$, p. adj $=0.074$

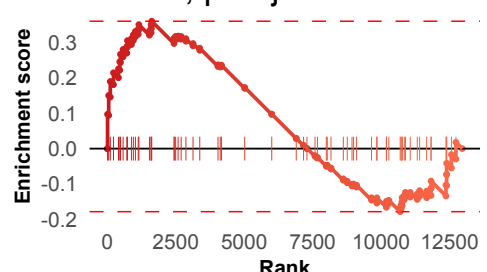

$\mathrm{NES}=-0.9, \mathrm{p} \cdot \mathrm{adj}=0.62$

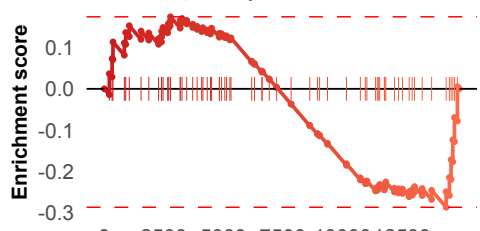

o 2500500075001000012500

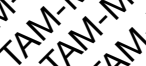
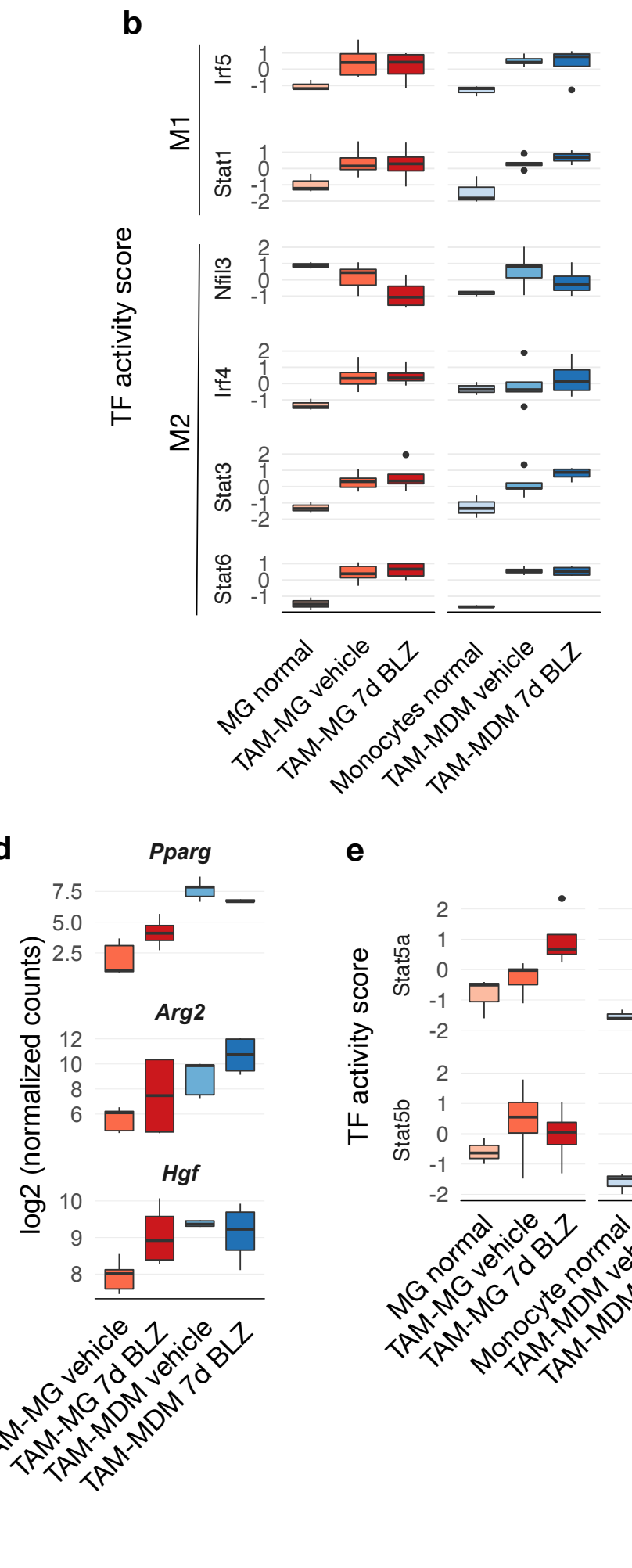

e

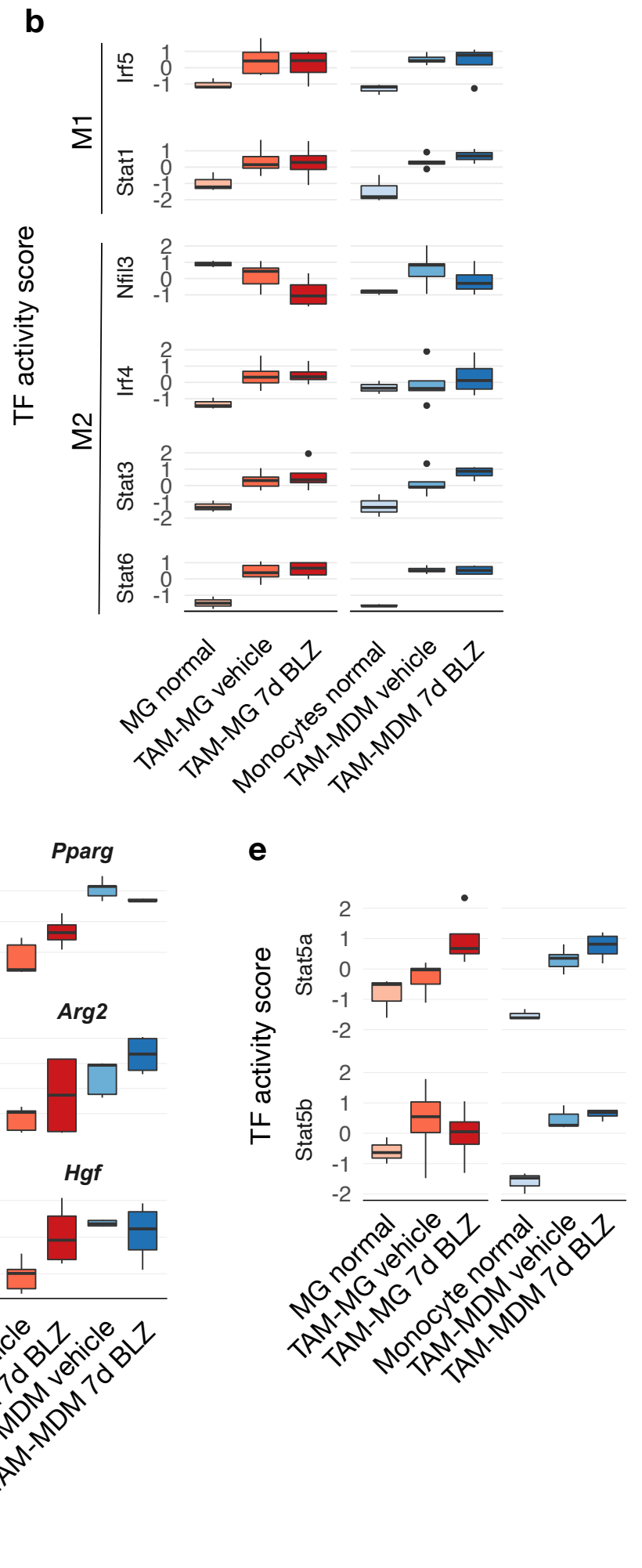

Pparg

pr g

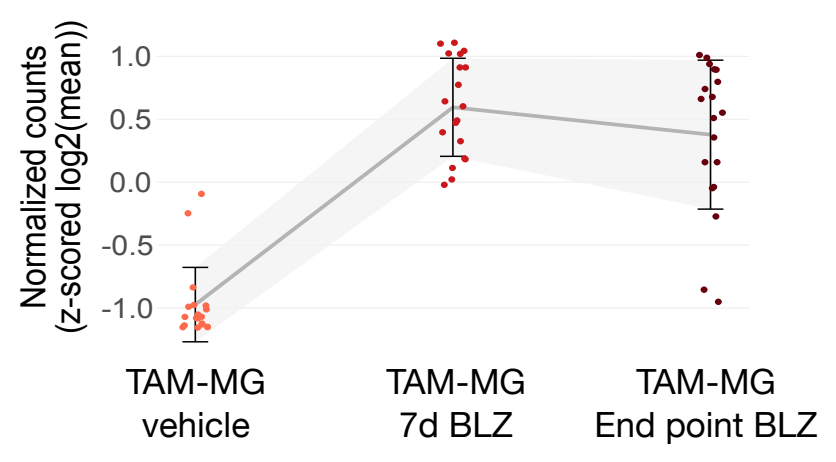


Fig. 6 CSF1R inhibition induces compensatory CSF2-mediated activation of TAMs.

a, Expression heatmap of M1- and M2-like macrophage markers in vehicle and 7d BLZ945 treated TAMs ( $n=5$; vehicle, $n=4$; BLZ945) from the MDA-BrM model and control monocytes or normal MG ( $n=3$ each). b, Transcription factor (TF) activity for a selected panel of M1/M2 associated transcription factors. c, Gene set ORA to identify pathways (top 20 based on their p-adjust, from the MSigDB C2 collection) that are induced in 7d-BLZ945 vs. Vehicle treated TAMs in MDA-BrM tumors ( $n=5$; vehicle, $n=4 ; B L Z 945$, fdr $\leq 0.1$ ). d, Box plots of CSF2 target gene expression and e, normalized TF activity scores for Stat5a and Stat5b in vehicle- and BLZ945-treated TAMs and normal MG and blood monocytes. f, GSEA of CSF1 and CSF2 signatures published in ${ }^{39}$ analyzed in 7d BLZ945-treated TAM-MG compared to vehicle. NES, normalized enrichment score. g, Z-scored normalized counts of CSF2 signature genes in TAM-MG in vehicle-, 7d- and end point-BLZ945 treated MDA-BrMs. 

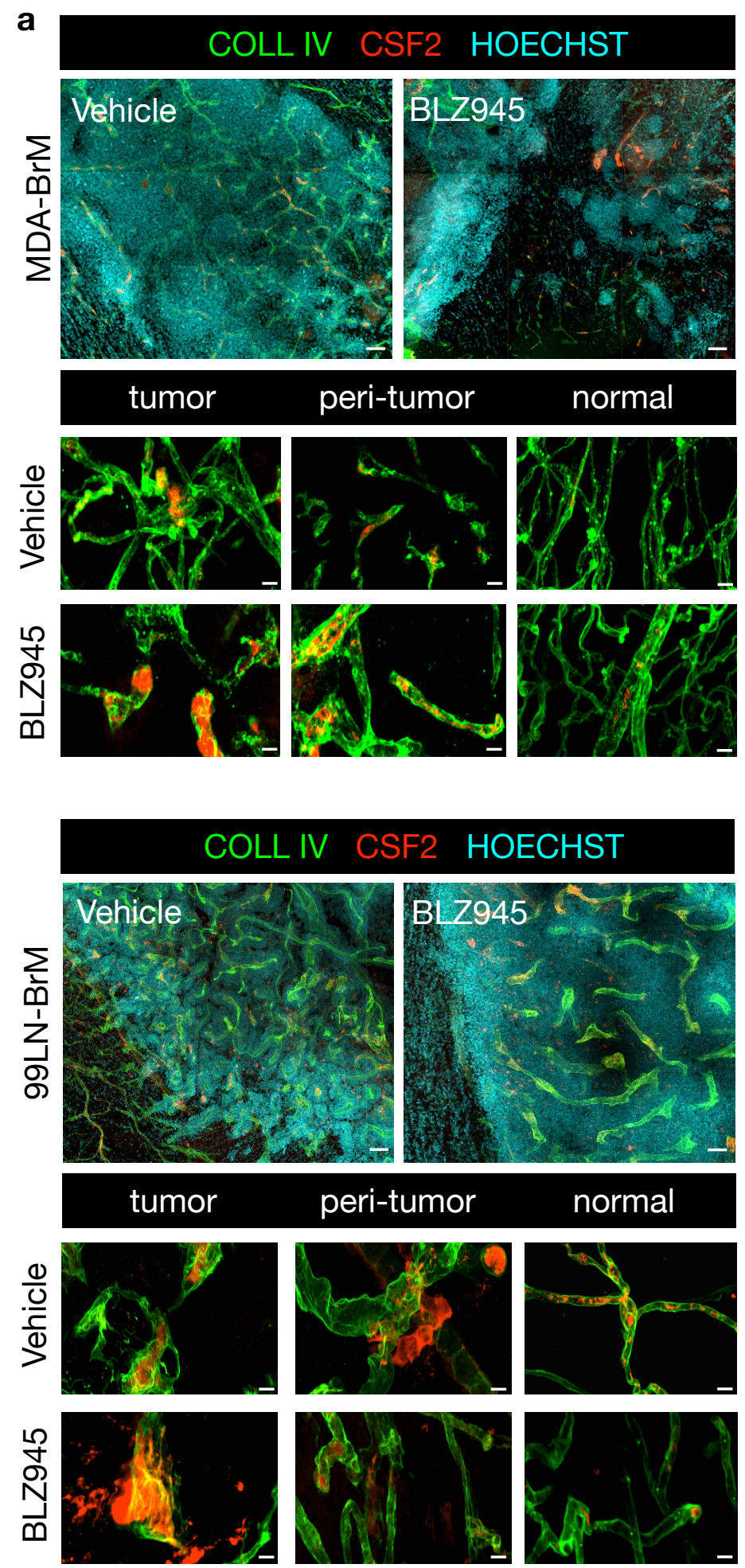
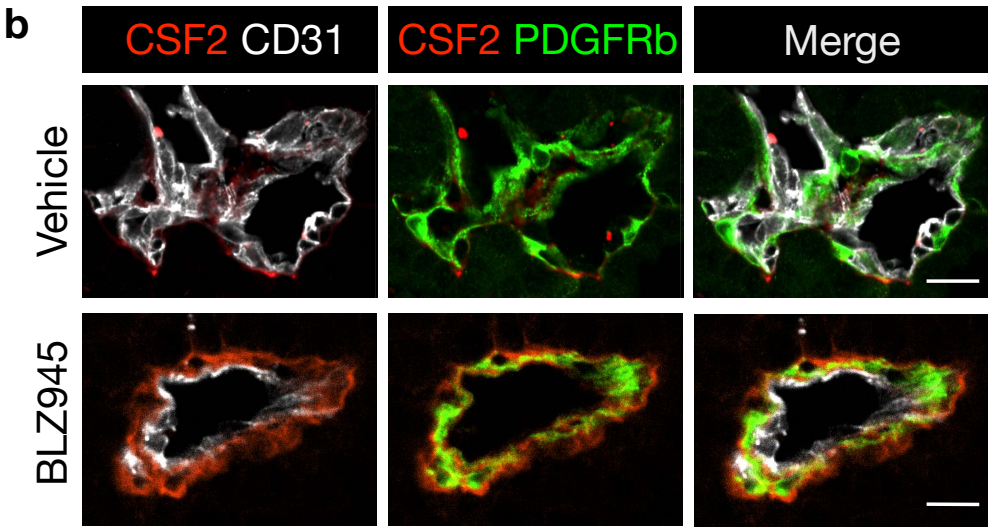

99LN-BrM

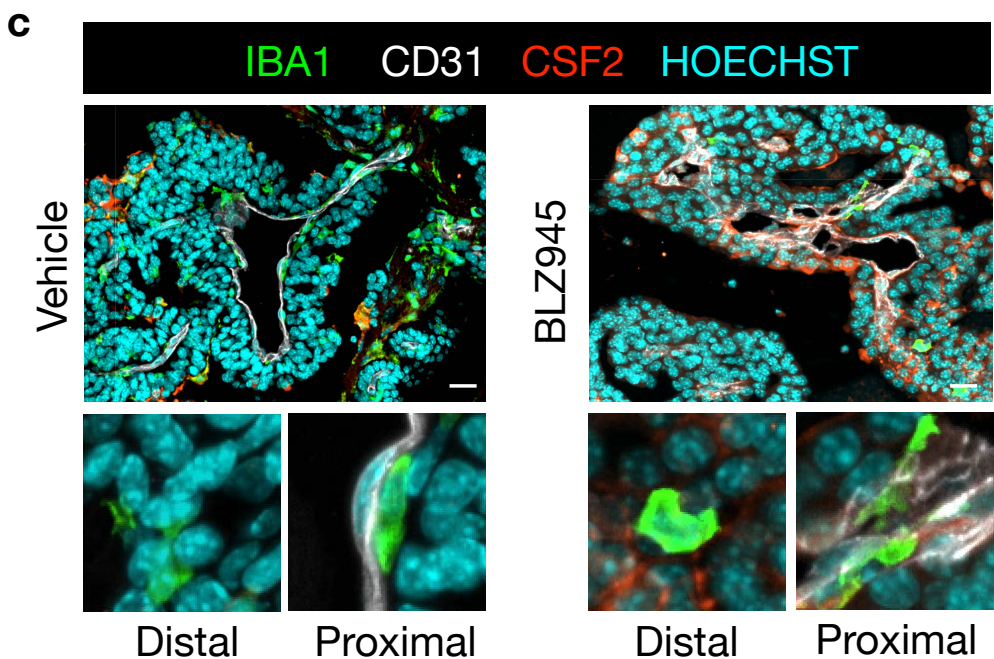

d

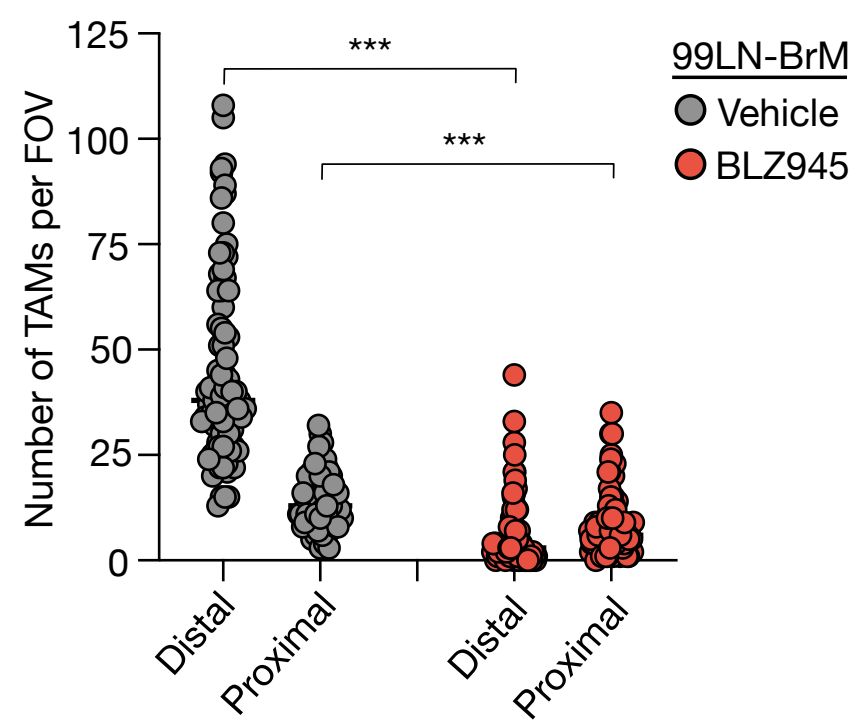


Fig. 7 CSF2-mediated TAM activation in perivascular protective niches.

a, Representative IF images of vehicle- and 7d BLZ945-treated MDA-BrM and 99LN-BrM brains stained for CSF2 (red) and collagen IV (COLL IV, green) to visualize vessels. HOECHST was used as nuclear counterstain. Scale bars; 50 and $10 \mu \mathrm{m}$. b, Representative IF images of vehicle- and 7d BLZ945-treated 99LN-BrM brains stained for CD31+ endothelial cells (white) and PDGFRb+ pericytes (green) in co-staining with CSF2 (red). c, Representative IF images of vehicle- and 7d BLZ945-treated 99LN-BrM brains stained for IBA1+ macrophages/microglia (green), CD31+ endothelial cells (white) and CSF2 (red) to visualize the localization of macrophages relative to the vascular niche. Scale bars; $25 \mu \mathrm{m}$. d, Quantification of macrophages/microglia proximal or distal to blood vessels as depicted in the representative images in vehicle and 7d BLZ945-treated animals ( $n=6$ per group with 15-20 FOV analyzed per animal). ${ }^{* * *} P<0.001$; two-tailed Student's t-test. 
a

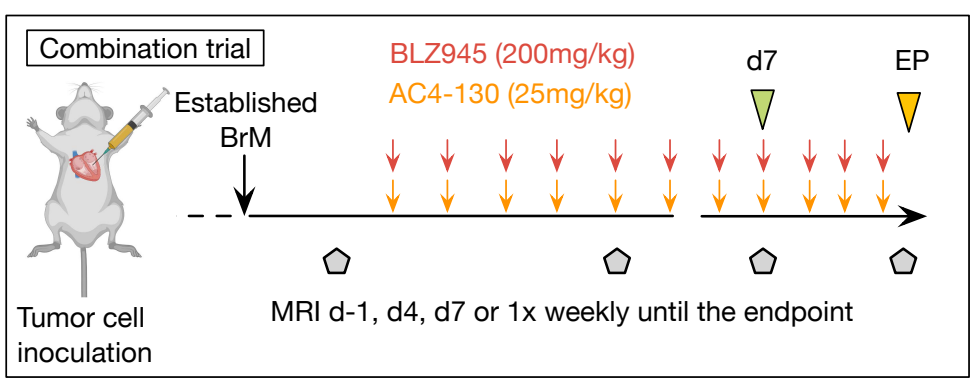

C
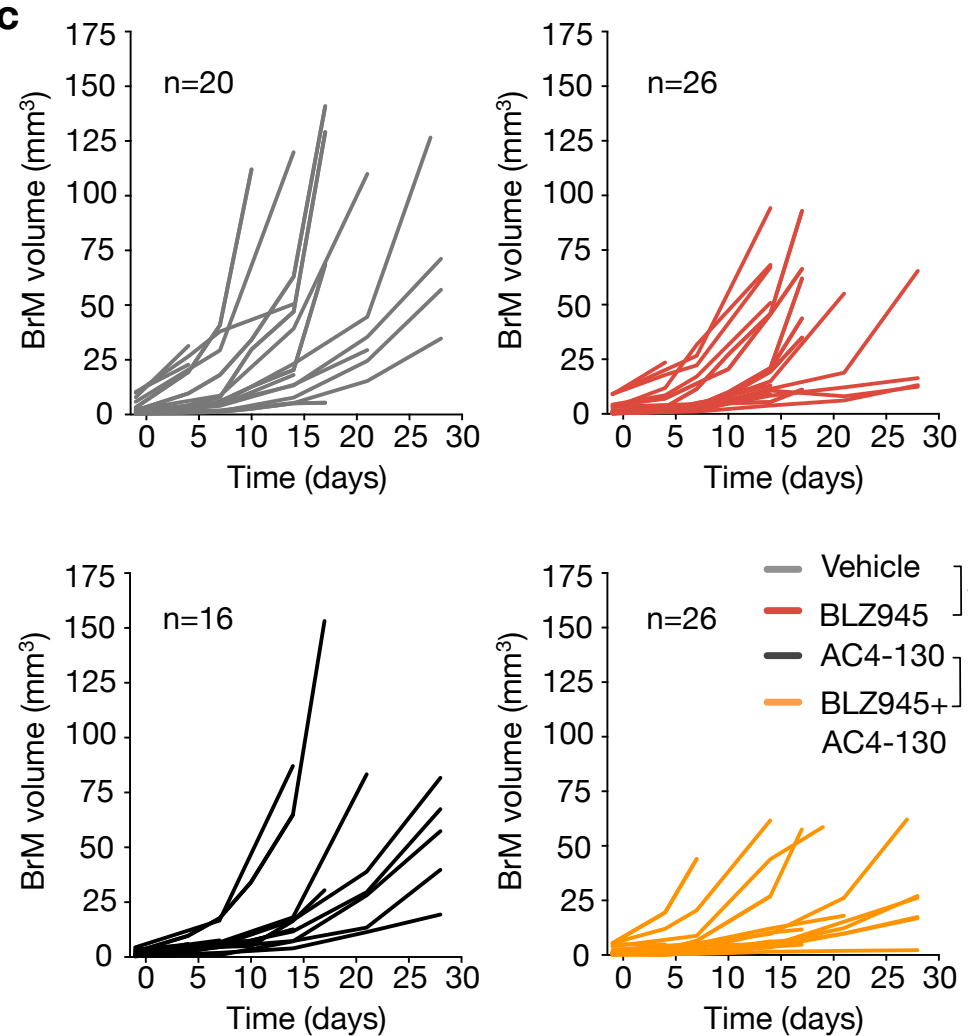

d

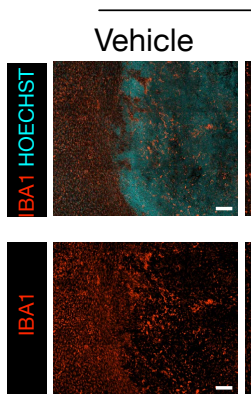

a
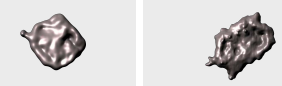

25
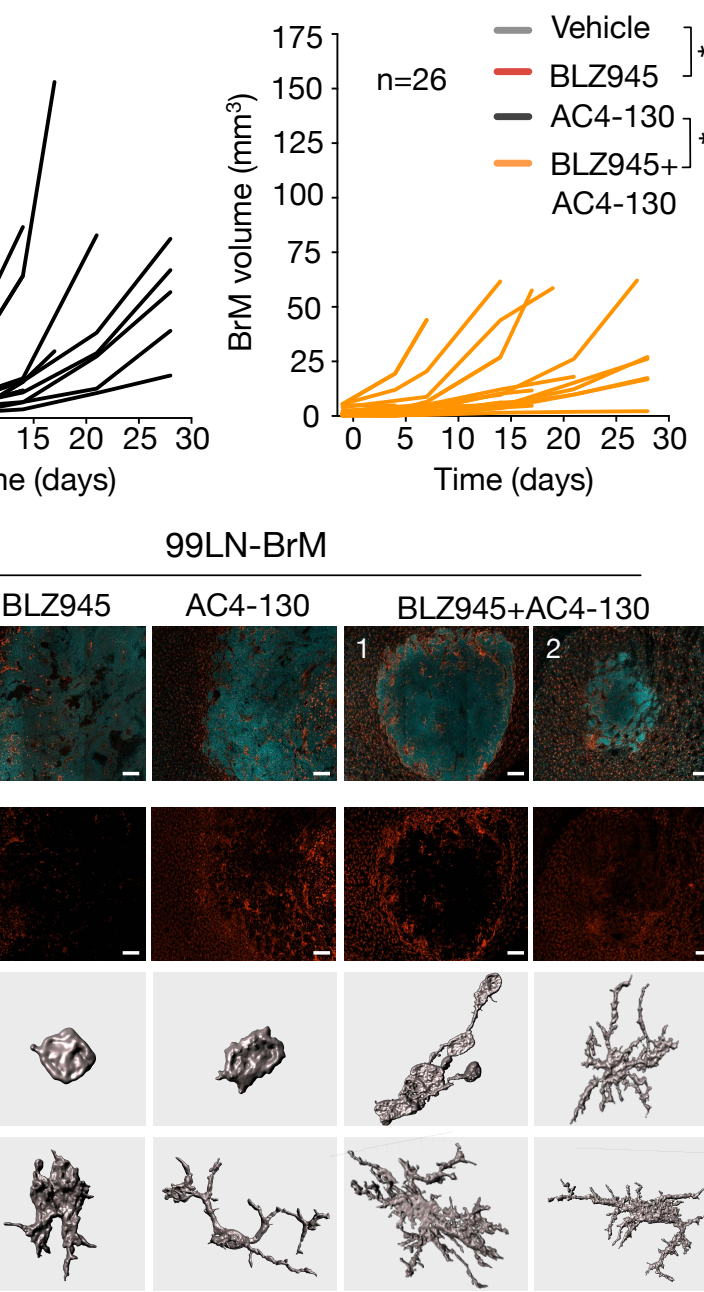

99LN-BrM
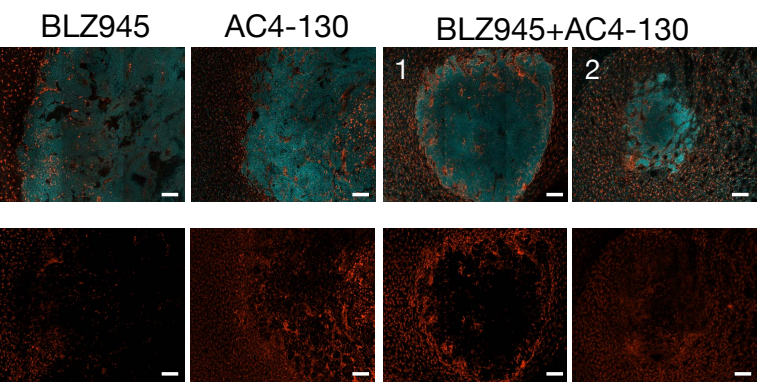

Adjacent brain
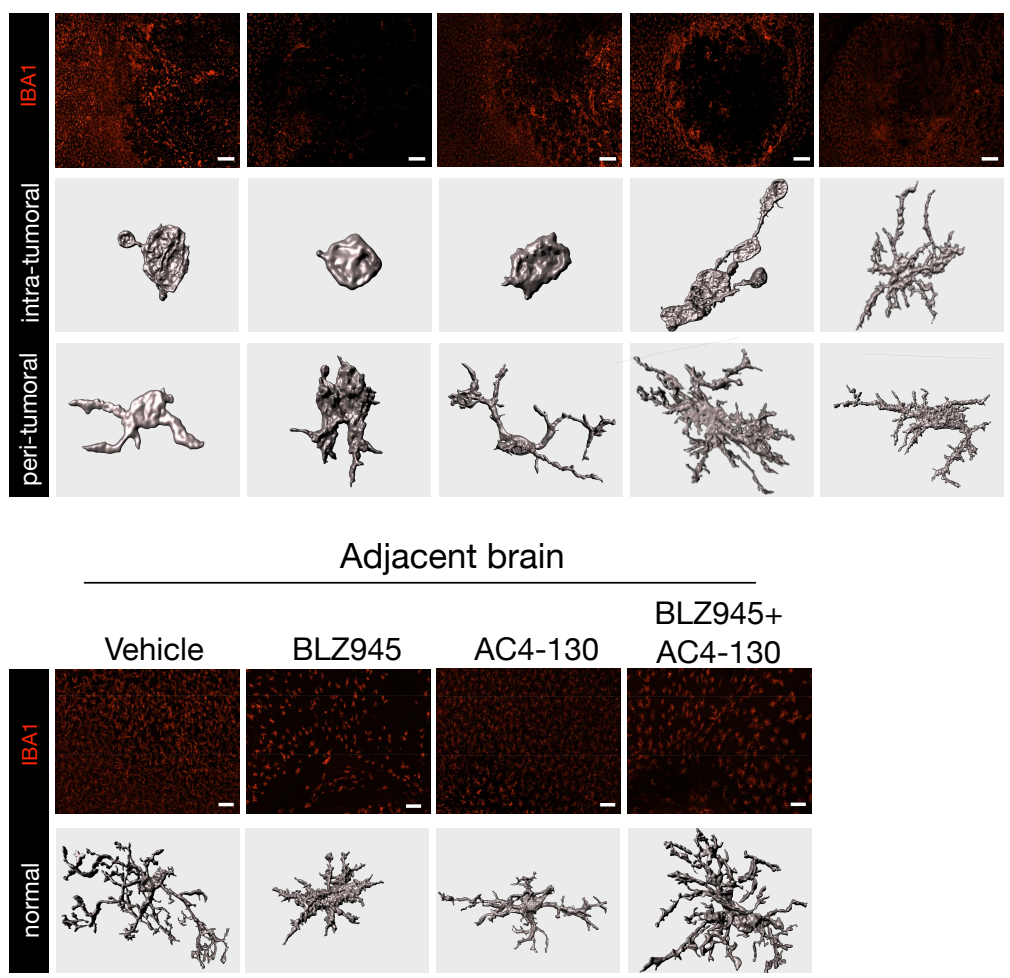
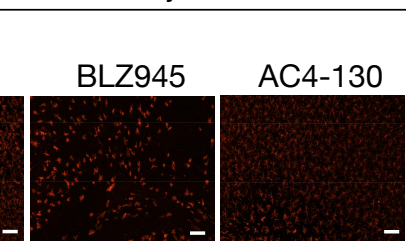

BLZ945+

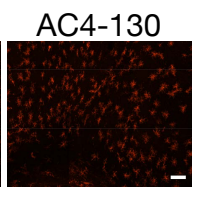

woris b
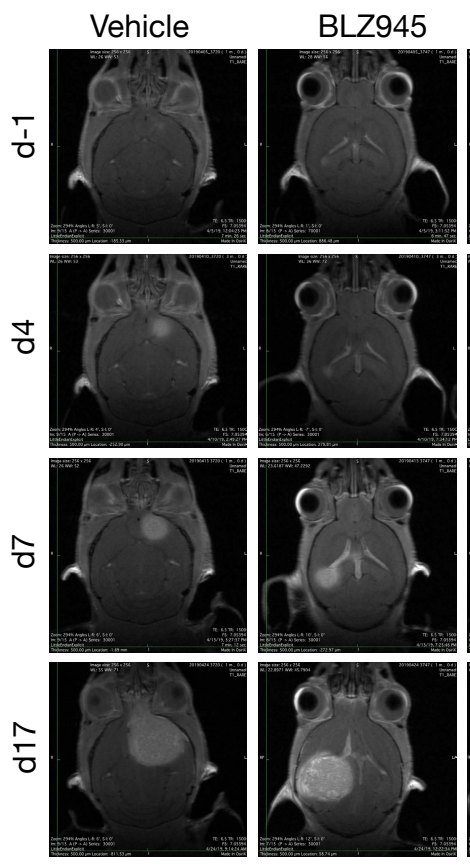

99LN-BrM

e
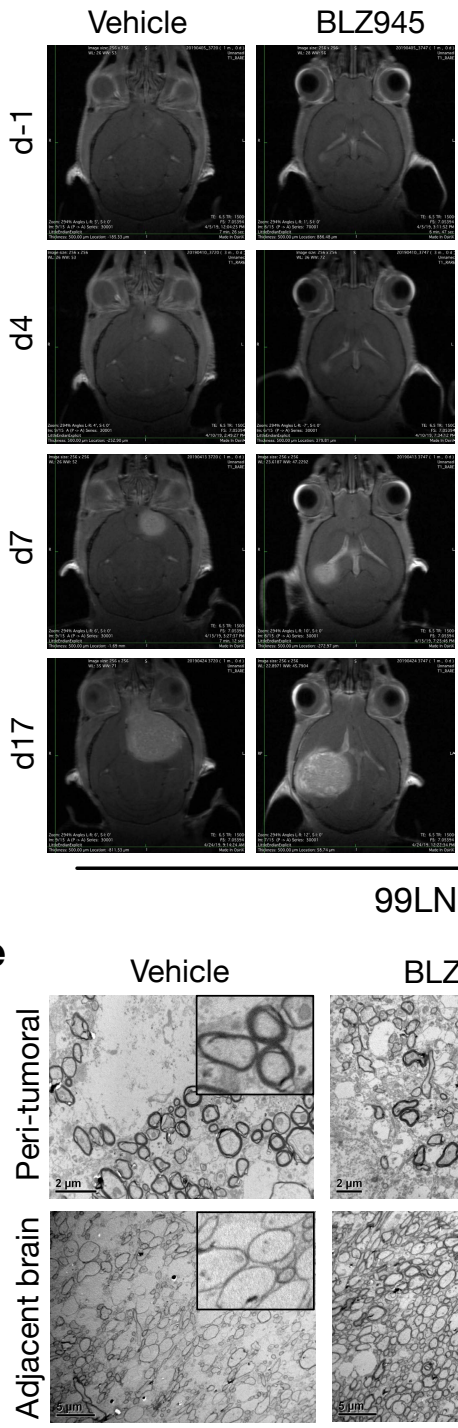

BLZ945
BLZ945+
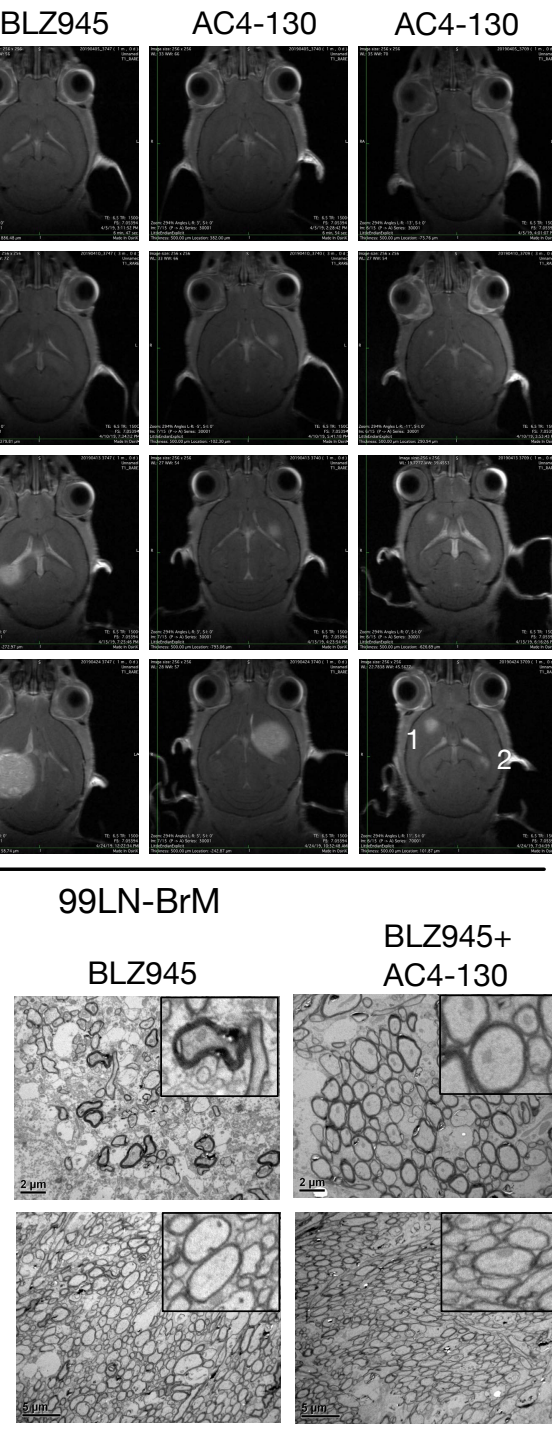

BLZ945+

AC4-130

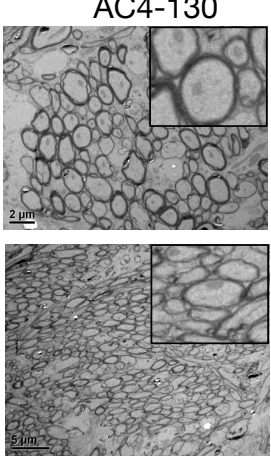

f Peri-tumor area
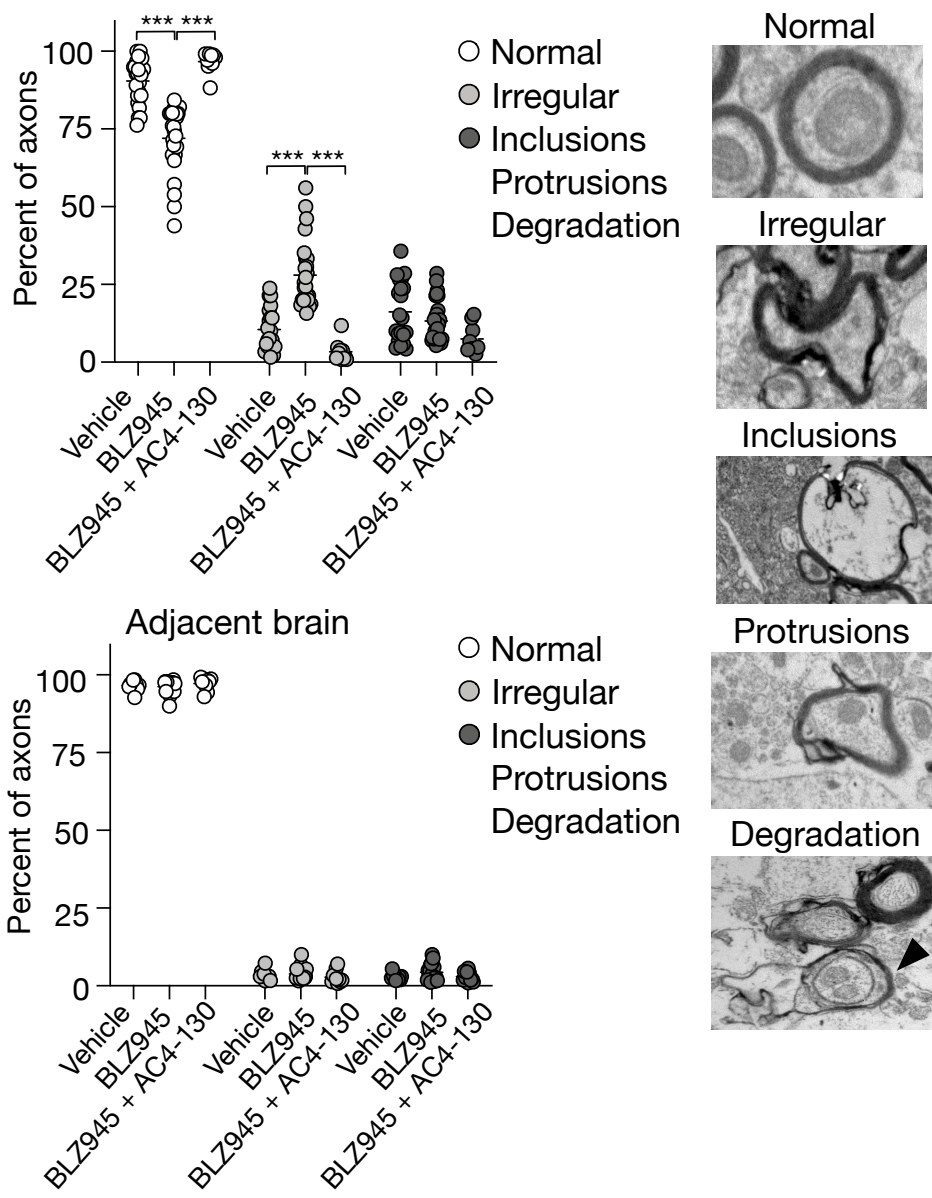

Protrusions
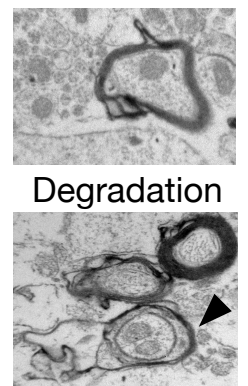
Fig. 8 Combined CSF1R and STAT5 inhibition leads to sustained anti-tumor control and normalization of TAM morphology.

a, Experimental design of the combination trial. b, Representative MRI images of 99LN-BrM mice at the indicated time points in response to treatment groups as illustrated in (a). Numbers 1,2 in the combination treatment group indicate lesions shown in panel $7 \mathrm{~d}$. c, Tumor growth curves for individual mice based on MRI volume $(n=20$; vehicle, $n=26$; BLZ945, $n=16$; AC4130, n=26; BLZ945+AC4130, ${ }^{*} P<0.05,{ }^{* *} P<0.01$, ${ }^{* * *} P<0.01$, two-tailed Student's t-test based on area under the curve. $\mathbf{d}$, Representative images and 3D reconstruction of IBA1+ cells in 99LN-BrM brains. Scale bars, 100 and $25 \mu \mathrm{m}$. e, Representative transmission electron microscopy images showing axons in the peri-tumor area (upper panel) and adjacent normal brain (lower panel) in the indicated experimental groups. Scale bar, $2 \mu \mathrm{m}$. f, Quantification of the percentage of normal and abnormal axons in the indicated experimental groups in the peri-tumor area and in the adjacent normal brain ( $n=2$ per group with 10 FOV analyzed per animal, ${ }^{* *} P<0.001$, two-tailed Students t-test). Abnormal axons were further classified into axons with irregular morphology (irregular) and those showing inclusions and protrusions or disaggregation of the myelin sheath indicative for degradation based on features depicted in the representative images. 
Klemm et al., Extended data Fig. 1
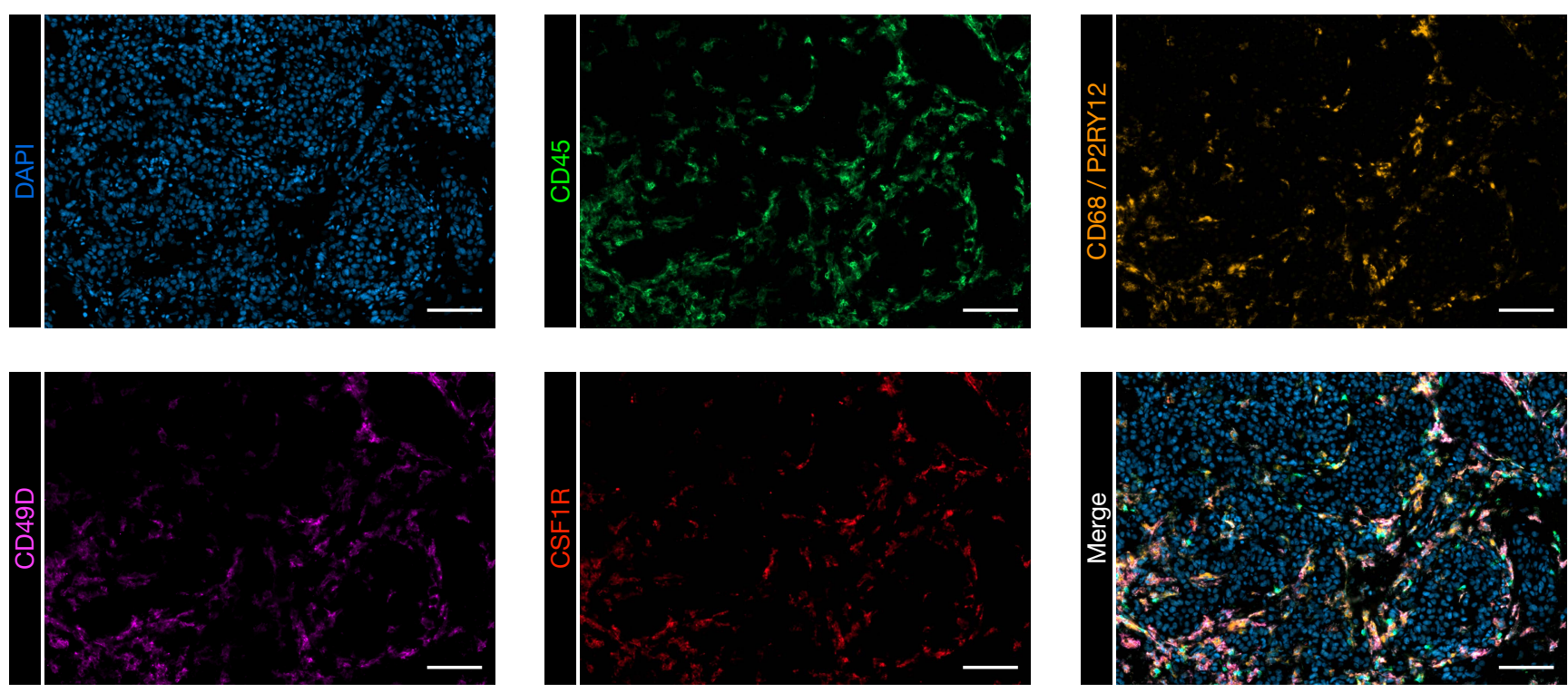


\section{Extended data Fig. 1 CSF1R expression in human BrM.}

Representative images of single channel and merged immunofluorescence (IF) images of CD45, CD68/ P2RY12, CD49D and CSF1R stainings used to identify CSF1R expression in different cell populations. Scale bar, $100 \mu \mathrm{m}$. 

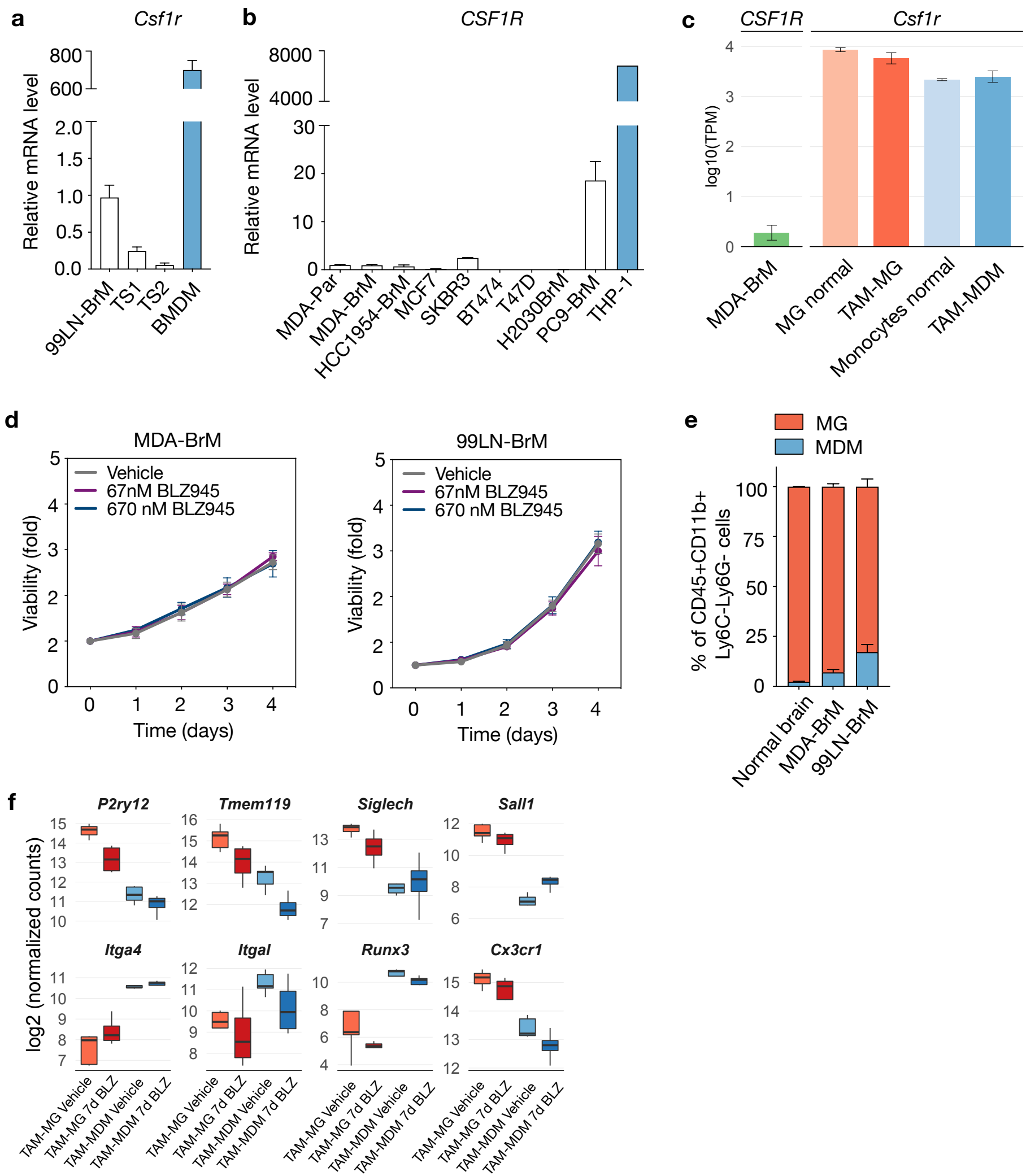

g

Non-TNBC

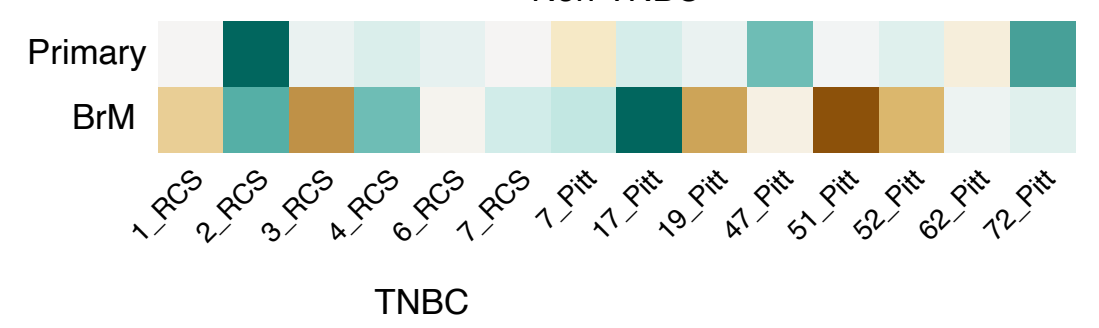

NES TAM

education genes

z-score

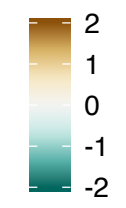

BrM 


\section{Extended data Fig. 2 CSF1R/Csf1r expression in mouse BrM.}

a,b, Quantification of CSF1R/Csf1r expression by qRT-PCR in (a) mouse and (b) human tumor cell lines in comparison to primary bone marrow-derived macrophages (BMDM) and the macrophage cell line THP1 ( $\mathrm{n}=3-9$ replicates). c, Quantification of CSF1R/Csf1r expression levels by RNAseq in FACS-purified MDA-BrM tumor cells, tumor-associated microglia and macrophages (TAM-MG and TAM-MDM) as well as normal microglia and blood monocytes isolated from tumor-free mice ( $n=5$ for tumor-bearing mice and $n=3$ for tumor-free controls). TPM, transcripts per million. $\mathbf{d}$, Measurement of cell viability in MDA-BrM and 99LN-BrM cells in response to BLZ945 treatment (0 nM- vehicle, $67 \mathrm{nM}$ and $670 \mathrm{nM})$ using MTS assay $(\mathrm{n}=3$ replicates). e, Quantification of the percentage of CD49d- MG and CD49d+ MDM in the CD45+CD11b+Ly6C-Ly6G- live cell population ( $n=3$ for tumor-free controls $n=5$ for MDA-BrM and $n=4$ for 99LN-BrM mice). f, Marker gene expression in normal MG and blood monocytes as well as TAM-MG and TAM-MDM to validate the purity of the FACS sorted populations. $(n=5$; vehicle, $n=4 ; B L Z 945)$. g, Heatmap depicting the z-standardized normalized enrichment score (NES z-score) of the union of DEG identified as BrM-TAM genes in matched human primary breast cancer and BrM from non-triple negative (Non-TNBC; upper panel) and triple negative breast cancer (TNBC; bottom panel). RNAseq data for patient breast cancer and BrM samples was obtained from ${ }^{25}$. NES between primary tumors and BrM was assessed using a Wilcoxon signed-rank test $(P=0.021)$. 


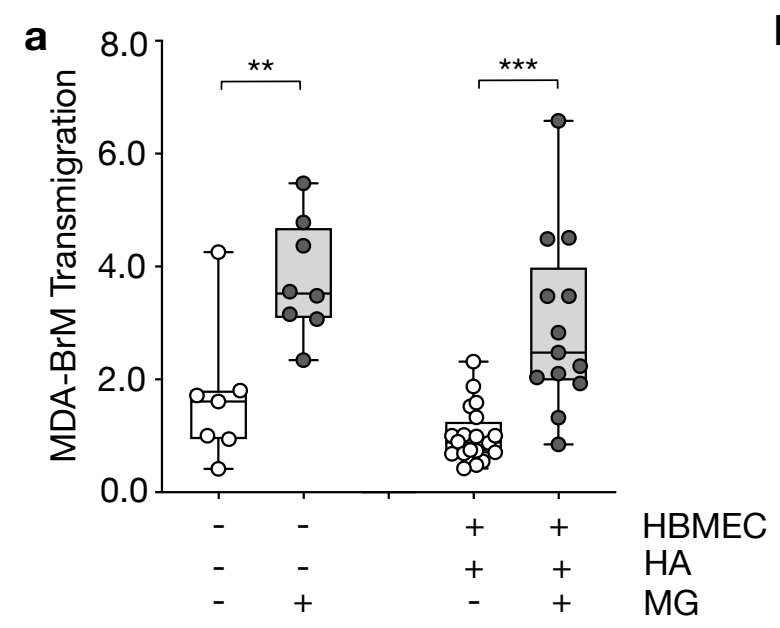

d

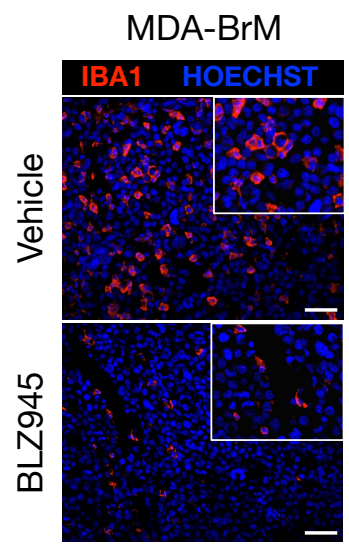

e

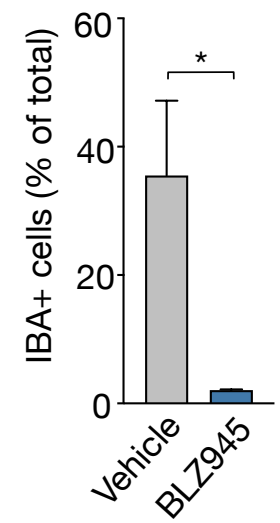

h

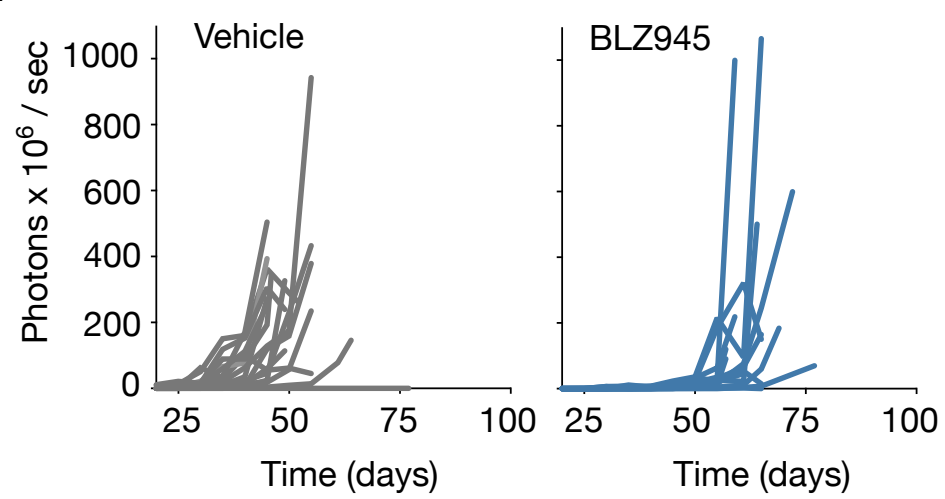

b
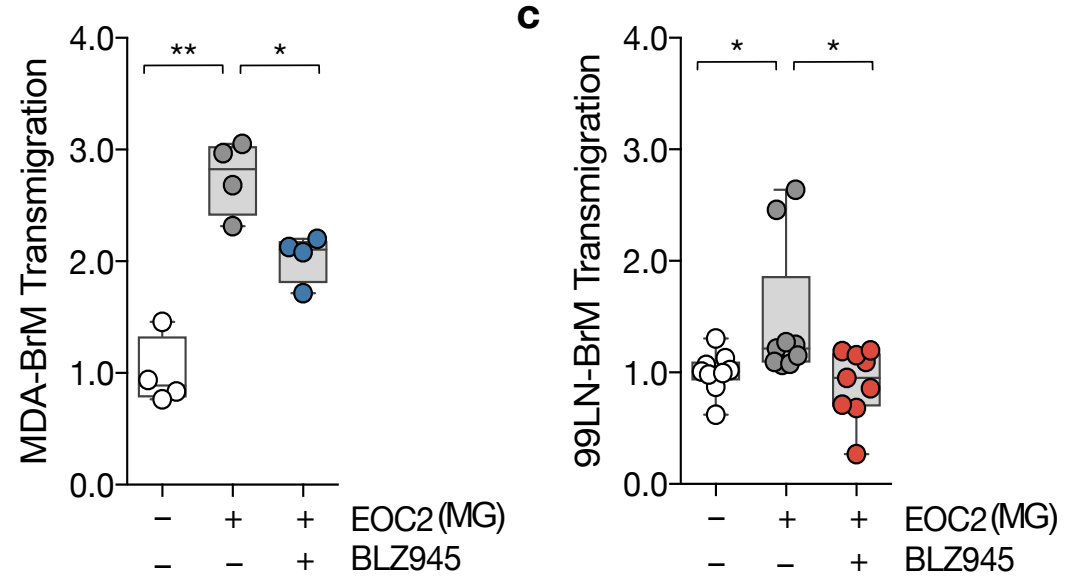

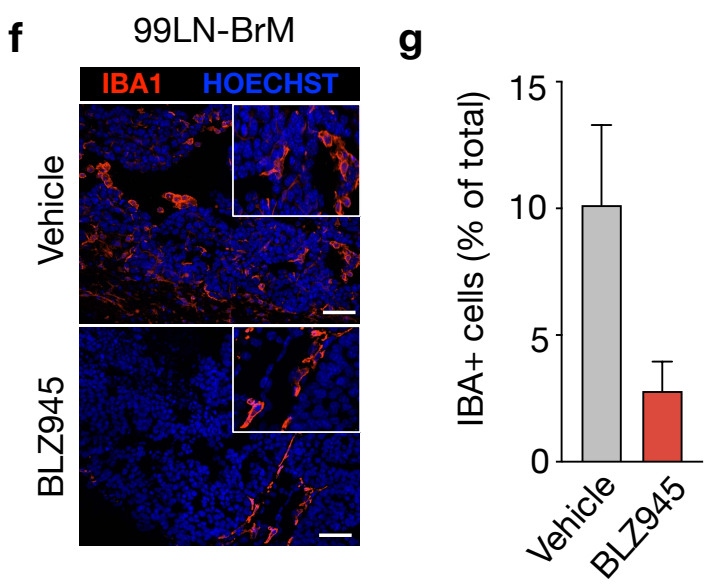

i
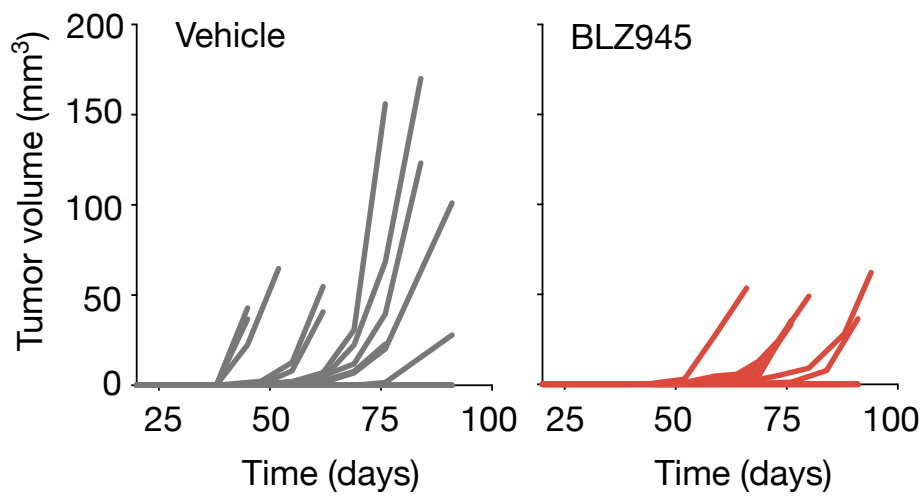


\section{Extended data Fig. 3 MG and BMDM support tumor cell extravasation.}

a, Quantification of the transmigration of MDA-BrM cells in the presence or absence of primary MG through an artificial blood brain barrier (BBB) formed by human astrocytes (HA) and human brain microvascular endothelial cells (HBMECs), or without an artificial BBB ( $n=7-11$ replicates per condition). Values are relative to the control condition. b, Quantification of the transmigration of MDA-BrM cells, with or without the addition of the microglia cell line EOC2, in response to BLZ945 or vehicle treatment ( $n=4$ replicates per condition). Values are relative to the control condition. c, Quantification of the transmigration of 99LN-BrM cells, with or without the addition of the microglia cell line EOC2, in response to BLZ945 or vehicle treatment ( $n=10$ replicates per condition). Values are relative to the control condition. $\mathbf{d}$, Representative immunofluorescence images of endpoint tumors from vehicle and BLZ945 treated MDA-BrM mice stained with IBA1 to visualize macrophages/microglia. HOECHST was used for nuclear counterstain. Scale bar, $50 \mu \mathrm{m}$. e, Quantification of the number of IBA1+ TAMs in vehicle and BLZ945 treated animals in MDA-BrM ( $n=5$ for each condition). f, Representative immunofluorescence images of endpoint tumors from vehicle and BLZ945 treated 99LN-BrM mice stained with IBA1 to visualize macrophages/microglia. HOECHST was used for nuclear counterstain. Scale bar, $50 \mu \mathrm{m}$. g, Quantification of the number of IBA1+ TAMs in vehicle and BLZ945 treated animals in 99LN-BrM mice ( $n=3$ for each condition). $\mathbf{h}$, Quantification of BLI intensity during tumor progression in the MDA-BrM model in vehicle or BLZ945 treated animals in prevention trial setting $(n=17 ;$ Vehicle, $n=14 ; B L Z 945)$. i, Quantification of the tumor volume based on T1 weighted MRI images during tumor progression in the $99 \mathrm{LN}-\mathrm{BrM}$ model in vehicle or BLZ945 treated animals in the prevention trial setting $\left(\mathrm{n}=10\right.$; vehicle, $\mathrm{n}=11$; BLZ945). ${ }^{*} P<0.05,{ }^{* *} P<0.01,{ }^{* * *} P<0.001$; two-tailed Student's t-test. 
a

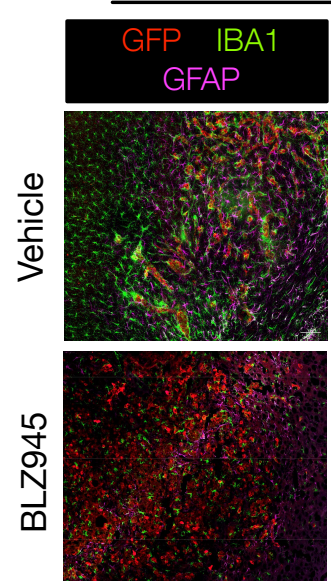

c

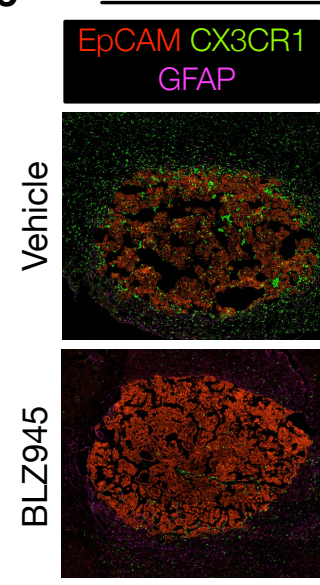

e
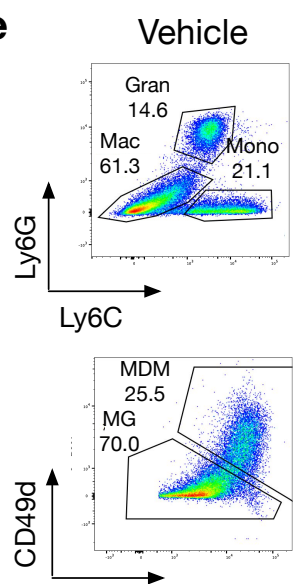

CD45
MDA-BrM
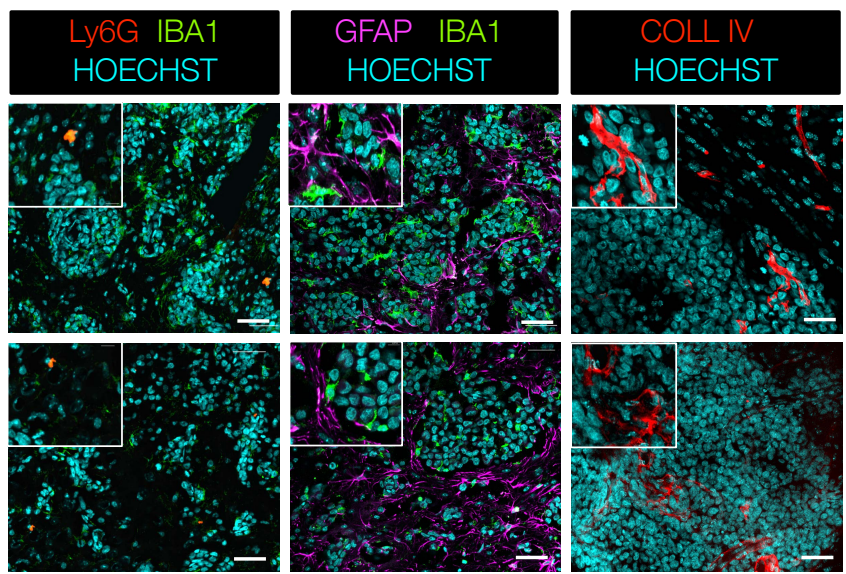

99LN-BrM
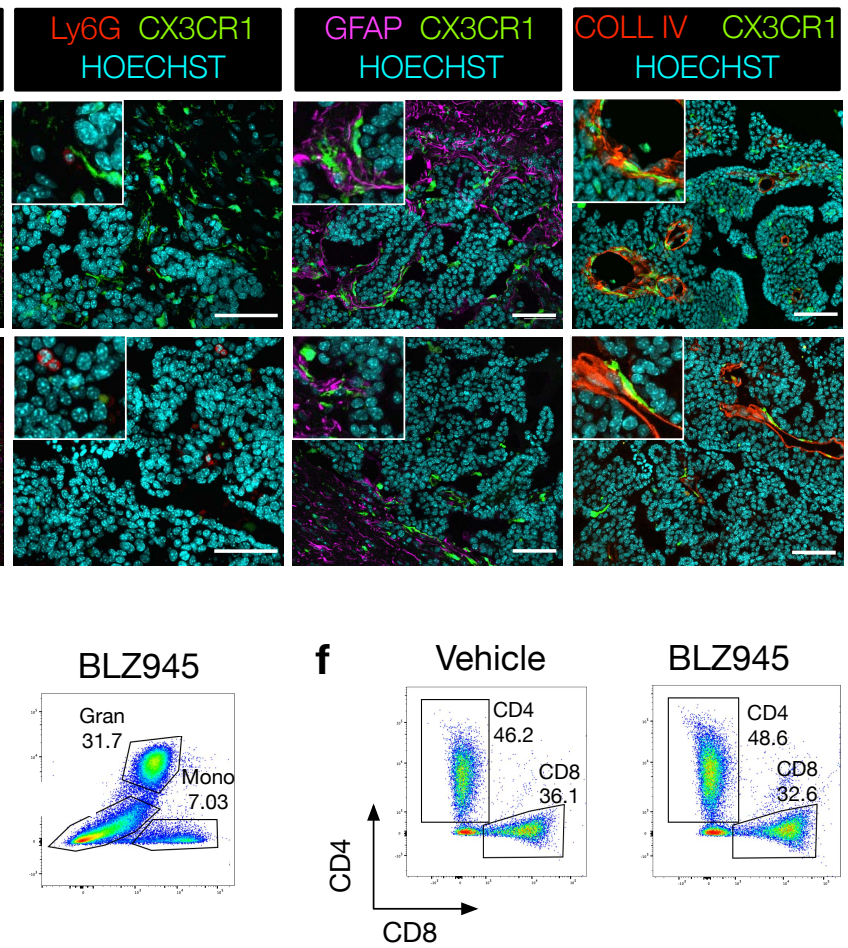

BLZ945
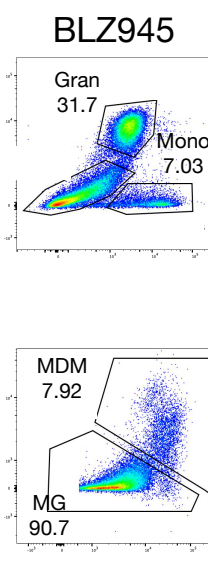

g

MDA-BrM
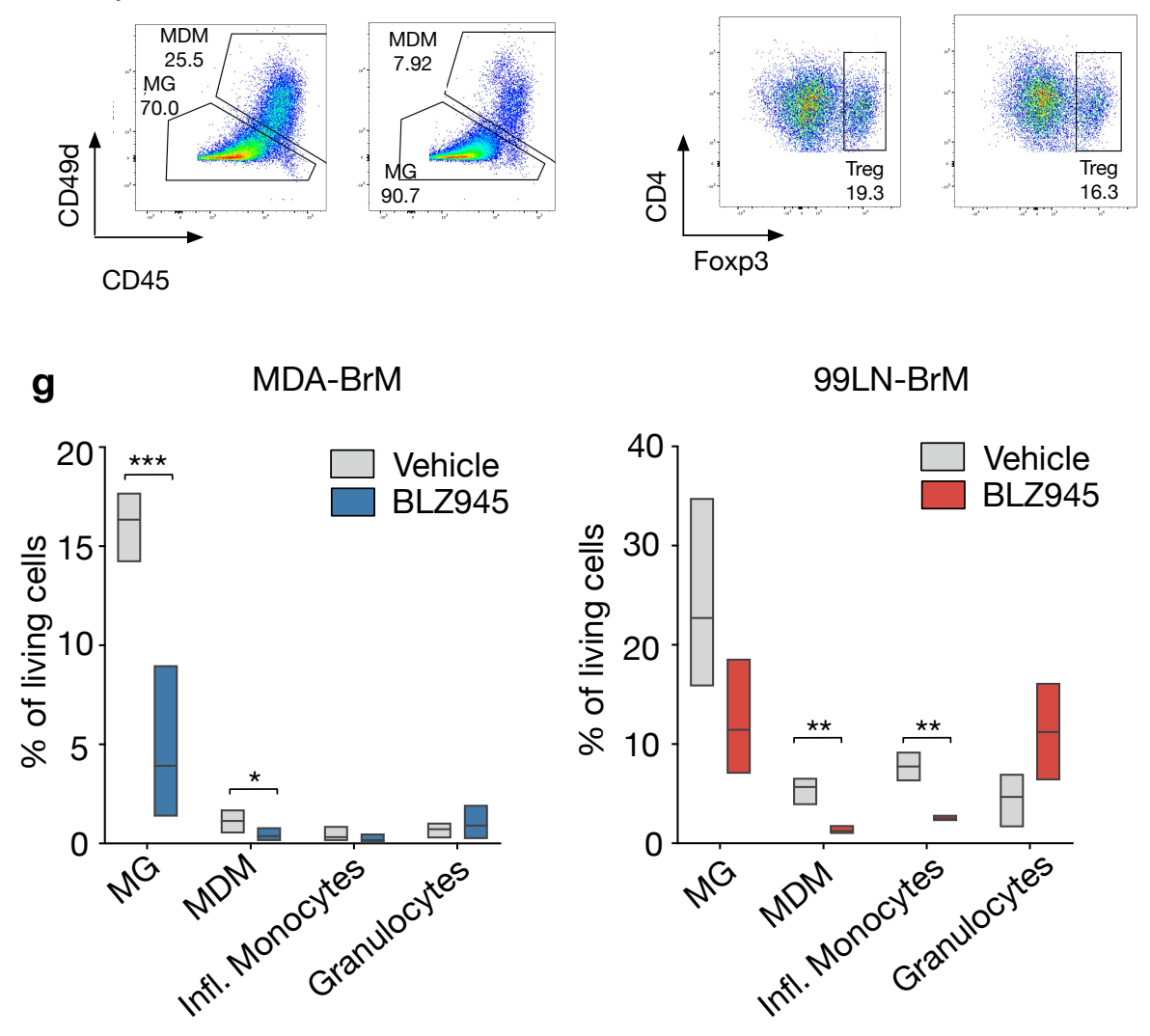

b

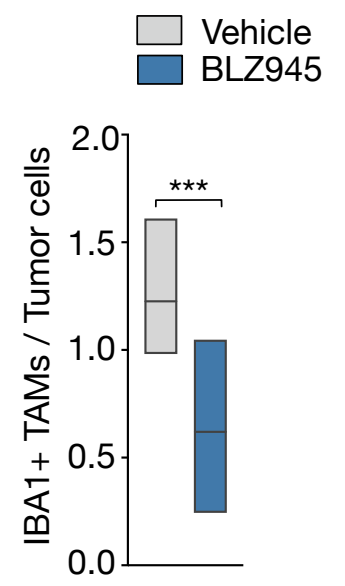

d

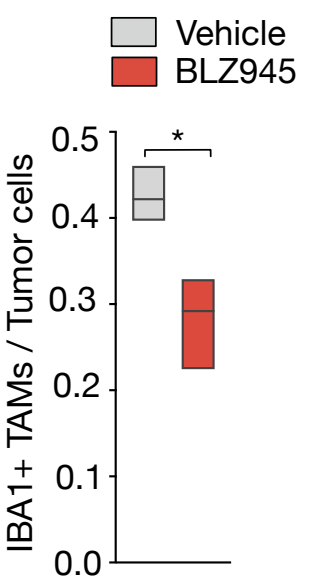

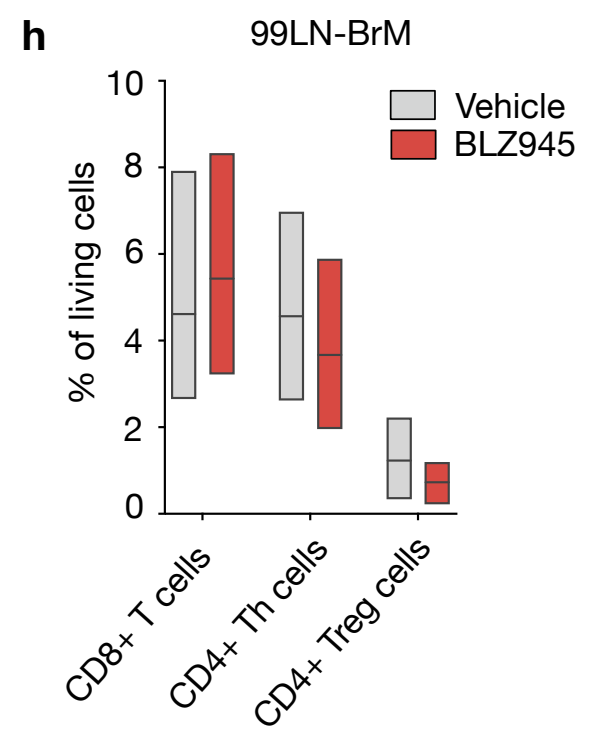




\section{Extended data Fig. 4 CSF1R inhibition leads to TAM depletion.}

a, Representative images of indicated markers in MDA-BrM brains after 7 days of vehicle or BLZ945 treatment. Scale bar, $50 \mu \mathrm{m}$. b, Quantification of TAMs in vehicle and BLZ945 treated MDA-BrM tumors ( $n=7$; vehicle, $n=7$; BLZ945). c, Representative images of indicated markers in $99 \mathrm{LN}-B r M$ brains after 7 days of vehicle or BLZ945 treatment. Scale bar, $50 \mu \mathrm{m}$. d, Quantification of TAMs in vehicle and BLZ945 treated 99LN-BrM mice $(n=3$; vehicle, $n=4$; BLZ945). e, Representative FACS plots of the myeloid panel used for flow cytometric analysis. f, Representative FACS plots of the lymphoid panel used for flow cytometric analysis. $\mathbf{g}$, Quantification of myeloid cell populations in the MDA-BrM ( $n=5$; vehicle, $n=4 ; B L Z 945)$ and 99LN-BrM model ( $n=3$; vehicle, $n=3$; BLZ945). $\mathbf{h}$, Quantification of lymphoid cell populations in the 99LN-BrM model ( $n=3$; vehicle, $n=3$ BLZ945). ${ }^{*} P<0.05$, ${ }^{* *} P<0.01,{ }^{* *} P<0.001$; two-tailed Student's t-test. 


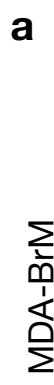

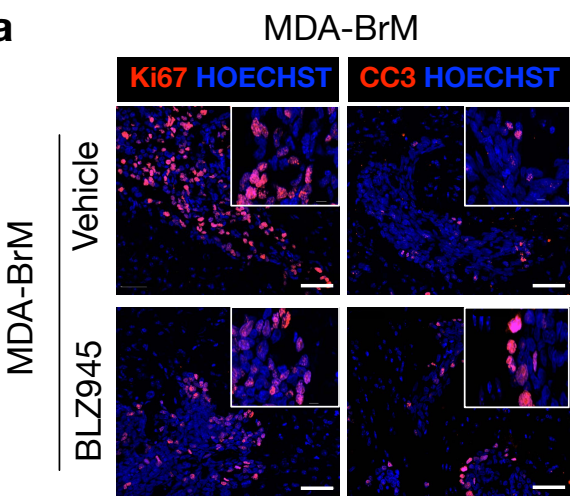

b

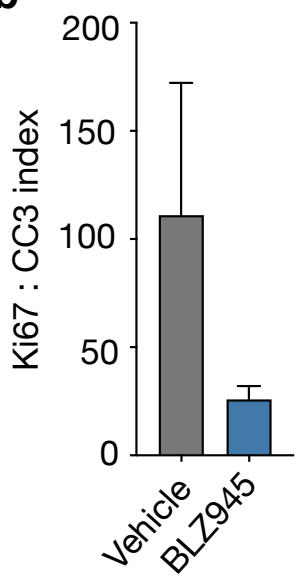

e

MDA-BrM
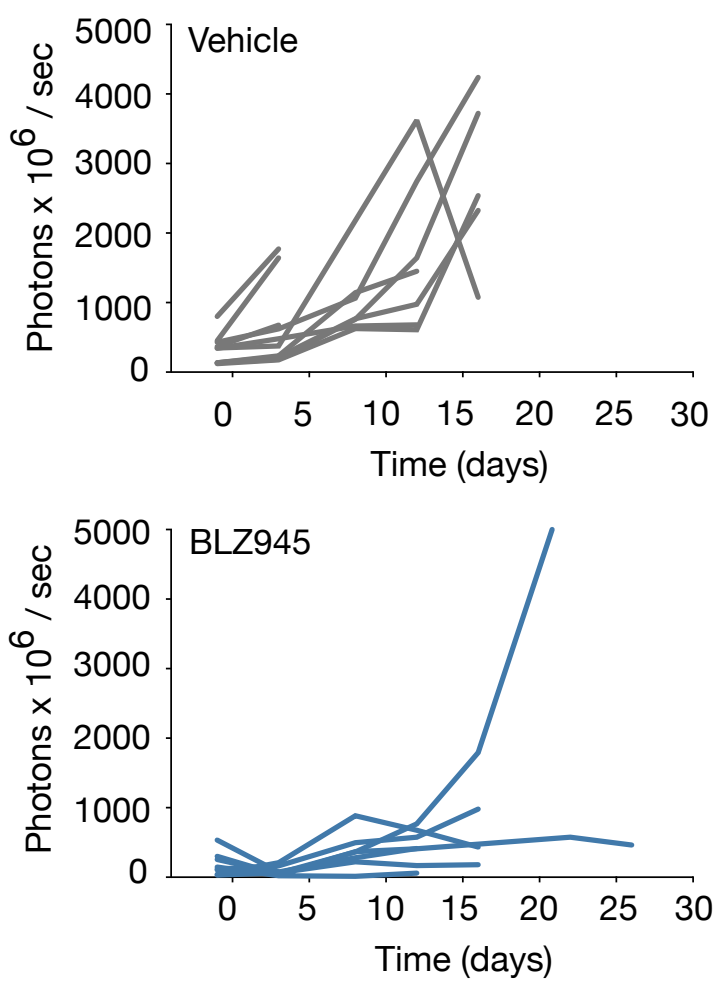

C
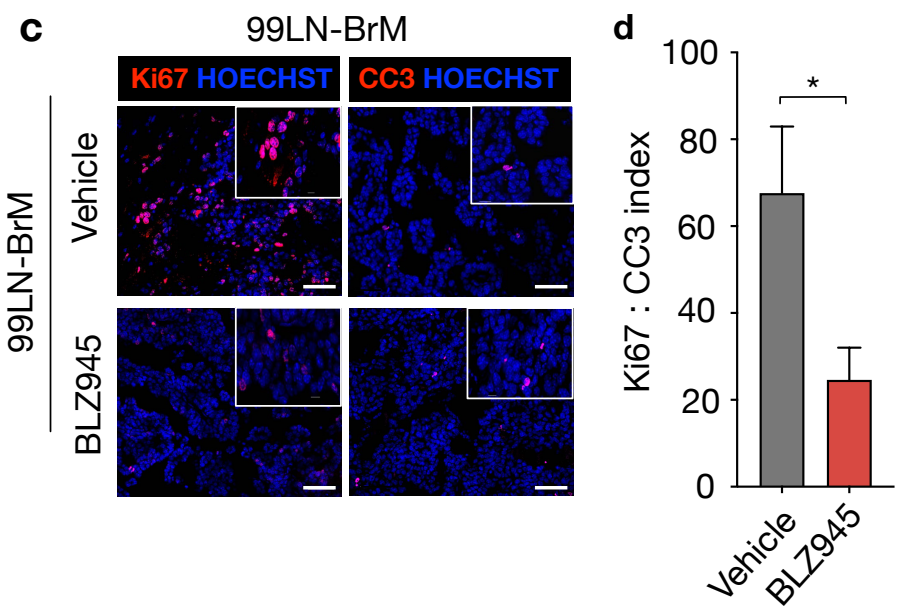

$\mathbf{f}$

99LN-BrM
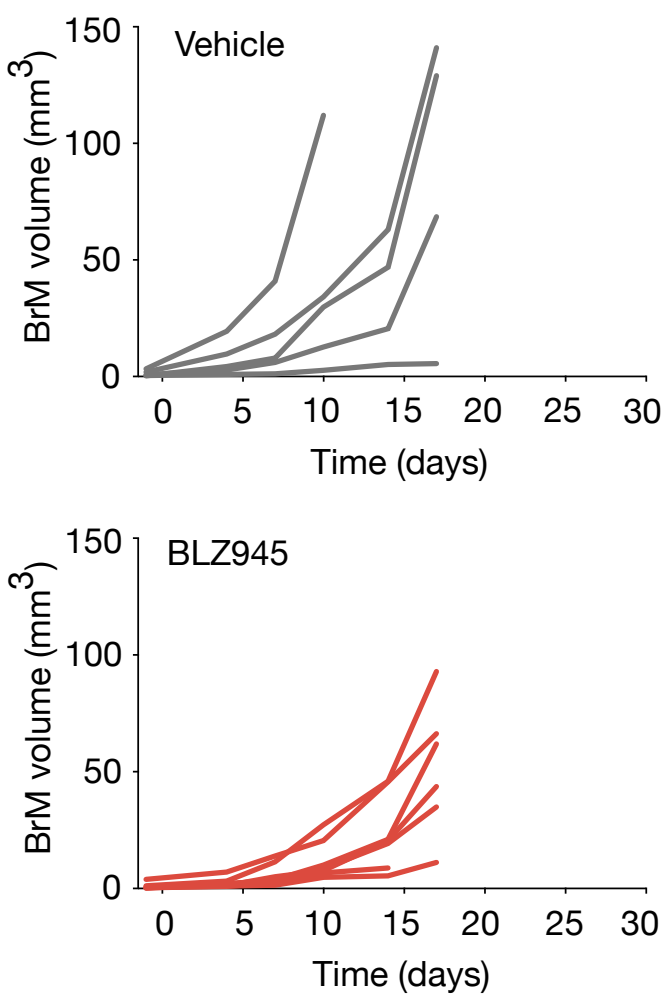
Extended data Fig. 5 BLZ945 treatment blocks tumor growth and reduces the proliferative index of tumor cells in intervention trials.

a, Representative immunofluorescence images stained for Ki67 (left panel) and cleaved caspase 3 (CC3, right panel) to visualize proliferating and apoptotic cells respectively in vehicle and BLZ945-treated mice from the MDA-BrM model. Scale bars, $50 \mu \mathrm{m}$. b, Ki67:CC3 proliferation:apoptosis index from immunofluorescence staining of vehicle and BLZ945treated MDA-BrM tumors ( $n=5)$. c, Representative immunofluorescence images stained for Ki67 (left panel) and cleaved caspase 3 (CC3, right panel) to visualize proliferating and apoptotic cells in vehicle and BLZ945-treated mice from the 99LN-BrM. Scale bar, $50 \mu \mathrm{m}$. d, Ki67:CC3 proliferation:apoptosis index from immunofluorescence staining of vehicle and BLZ945-treated 99LN-BrM tumors ( $n=3)$. e, Quantification of BLI intensity of vehicle and BLZ945-treated mice from the MDA-BrM model $(n=10$; vehicle and $n=8 ; B L Z 945)$. f, Quantification of the tumor volume based on T1 weighted MRI images during tumor progression in the 99LN-BrM model in vehicle or BLZ945 treated animals in the intervention trial setting ( $n=5$; vehicle, $n=7$; BLZ945). * $P<0.05$; two-tailed Student's t-test. 
a

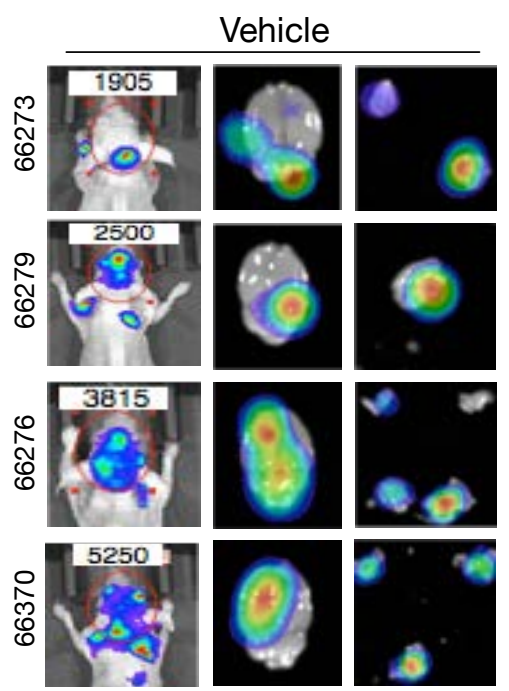

b
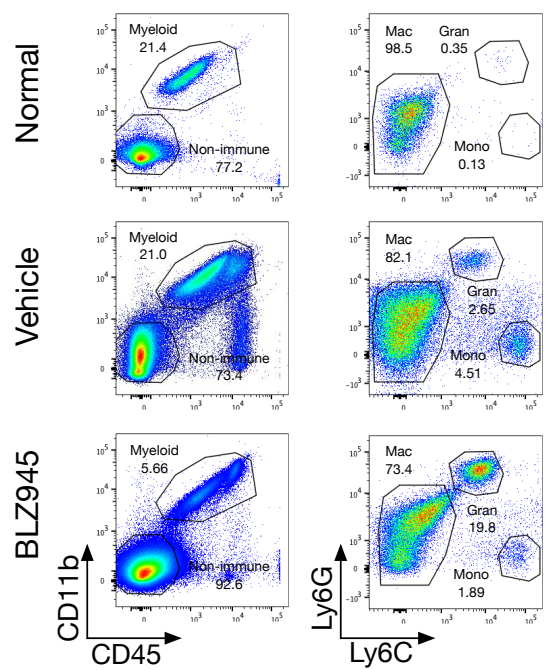

d

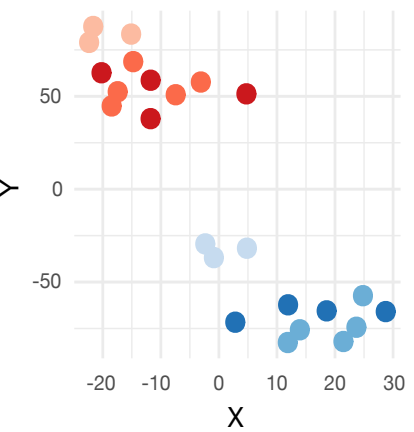

\section{Condition}

MG normal

Monocytes normal

TAM-MG Vehicle

TAM-MDM Vehicle

TAM-MG 7d BLZ
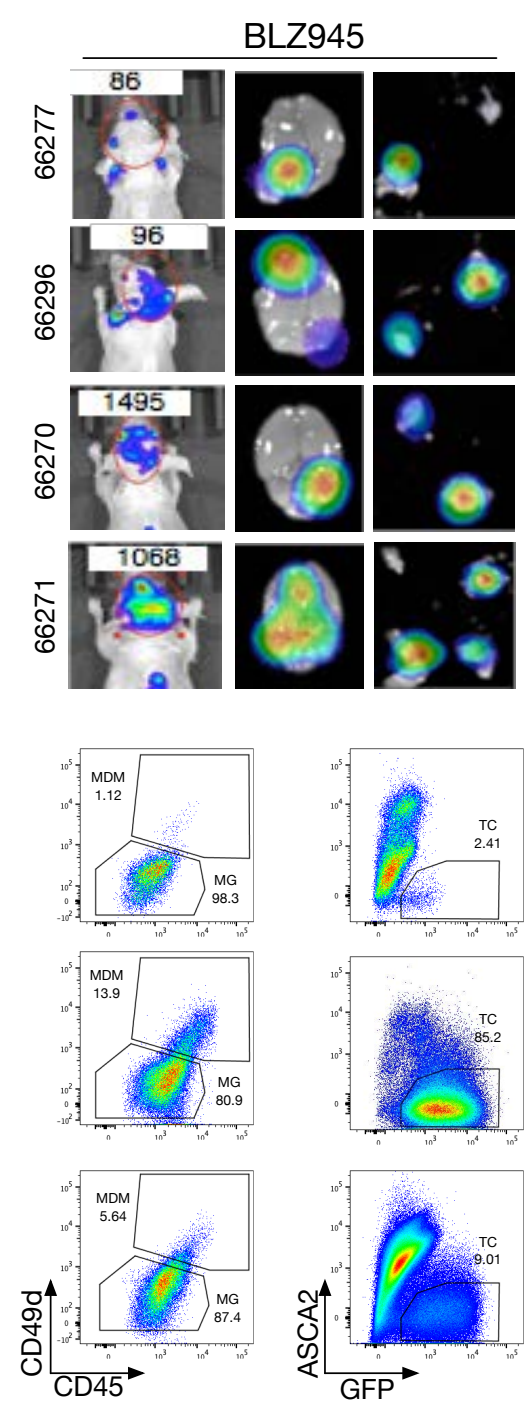

e

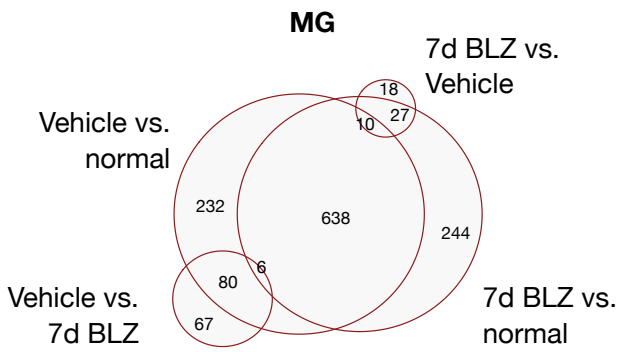

C

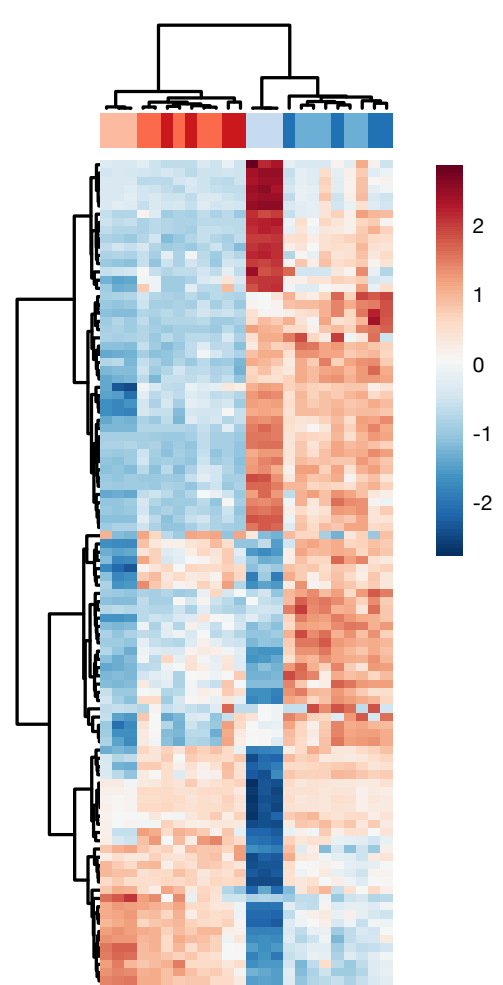

\section{Condition}

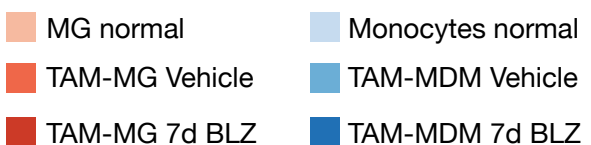

TAM-MG vs. normal

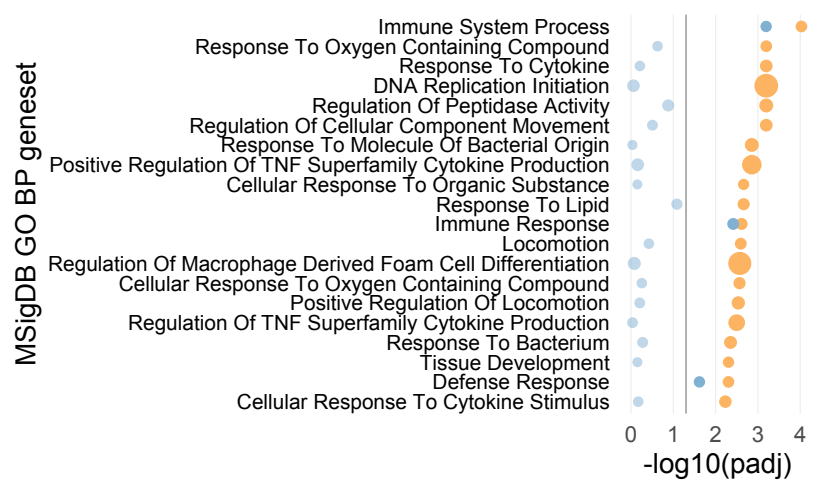

TAM-MDM vs. normal

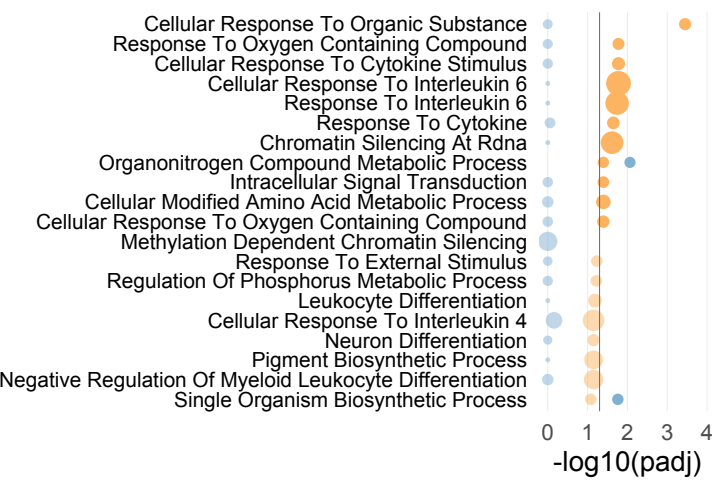

Condition

- TAM Veh TAM 7d BLZ

Fraction of gene set

- 0.1

0.2 7d BLZ vs. normal

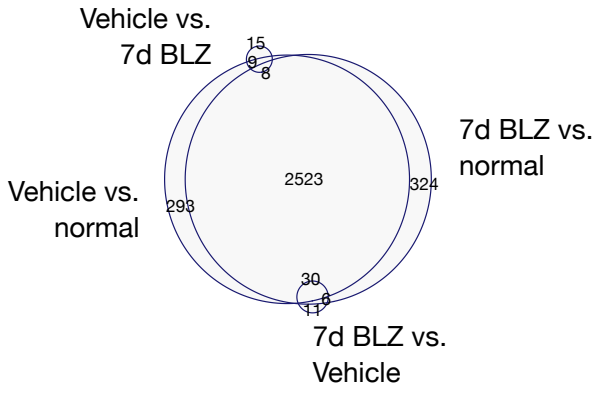




\section{Extended data Fig. 6 Differential gene expression in FACS-purified tumor cells and myeloid cell populations.}

a, Representative in vivo and ex vivo images of vehicle and BLZ945-treated MDA-BrM mice at $\mathrm{d} 7$ after treatment start. b, Representative FACS plots of the gating strategy for FACS purification of GFP+ tumor cells and TAM-MG (CD45+CD11b+Ly6C-Ly6G-CD49d-) and TAM-MDM (CD45+CD11b+Ly6C-Ly6G-CD49d+) from the MDA-BrM model used for RNAseq experiments. Tumor-free mice were used to isolate blood monocytes and normal MG. c, Heatmap of 100 most variant genes in myeloid populations across all samples. Columns and rows were clustered using Ward's method with 1 - Pearson's correlation coefficient as the distance measurement. d, tSNE plot on 2000 most variant genes shows clustering of individual samples. e, Euler plot depicts shared and unique DEG (p-adjusted<0.05, $\log 2 \mathrm{fc}>1$, mean expression > 10) in MG (right panel) and MDM (left panel) in the indicated comparisons. f, Gene set ORA (from the MSigDB GO collection) of DEGs that are specifically induced in MDA-BrM TAM-MG and TAM-MDM in response to CSF1R blockade compared to normal MG and blood monocytes (fdr $\leq 0.1)$. 
a
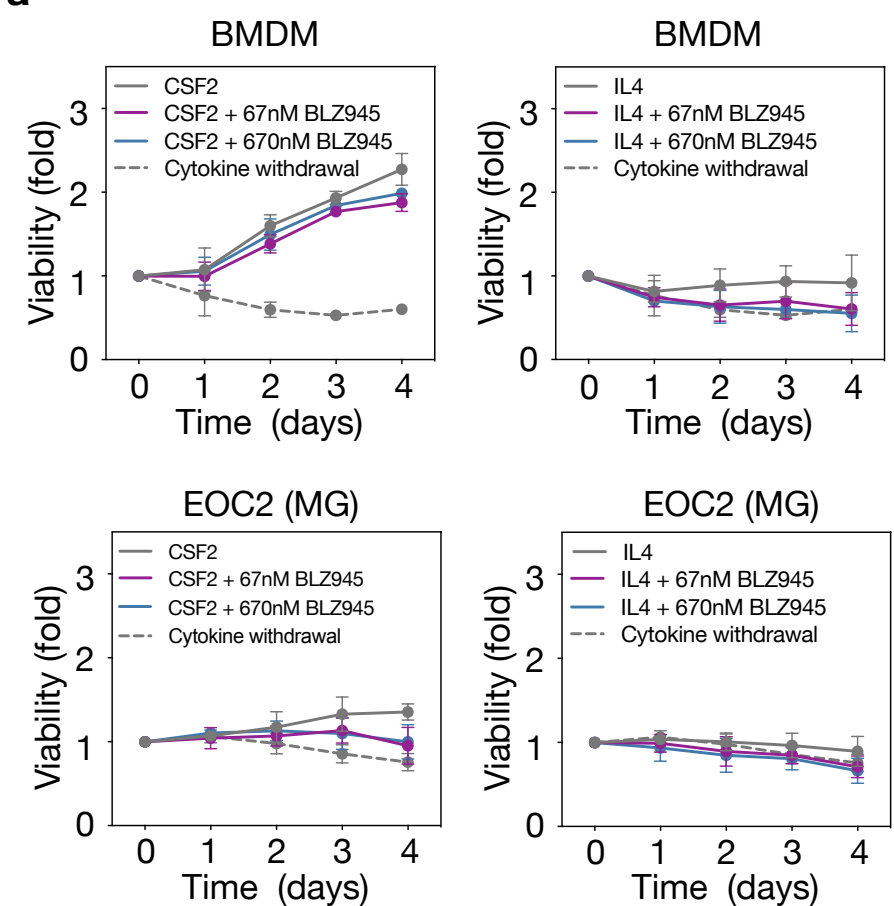

C

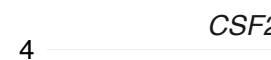

3

2

要

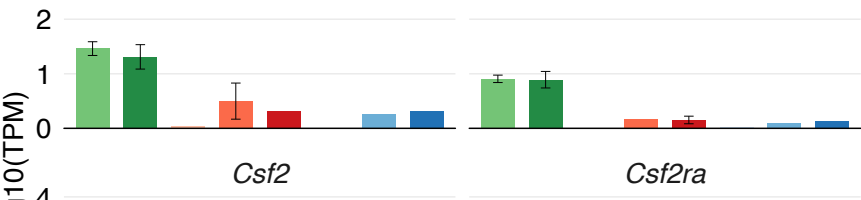

은 4

1 0

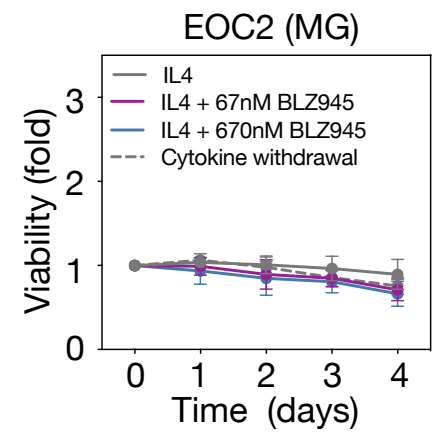

CSF2RA b
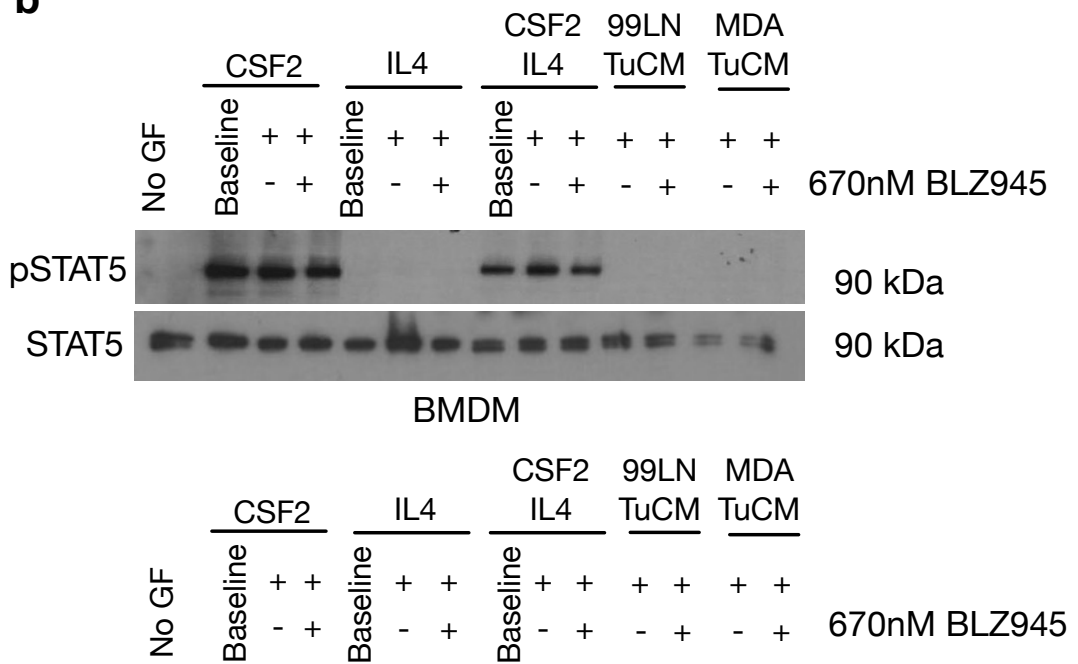

pSTAT5

STAT5

$---$

$90 \mathrm{kDa}$

$90 \mathrm{kDa}$

CSF2RB

Condition

MDA-BrM vehicle

- MDA-BrM BLZ

MG normal brain

Csf2rb

- TAM-MG vehicle

- TAM-MG BLZ

Monocytes normal

TAM-MDM vehicle

- TAM-MDM BLZ

\section{d CSF2 mediated neuro-inflammation}

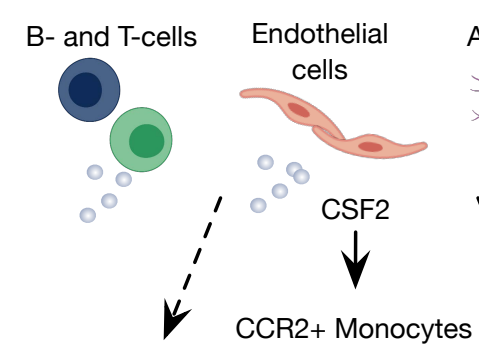

Neutrophils
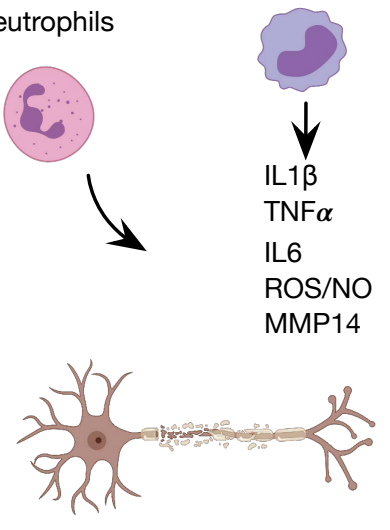
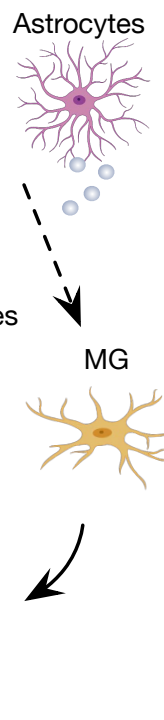

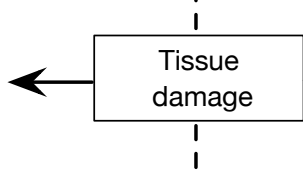

\section{CSF2 mediated inflammation in BrM}

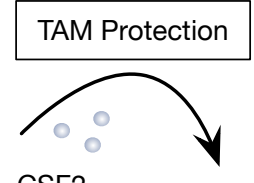

CSF2

CSF2

activated TAM

FSF1R

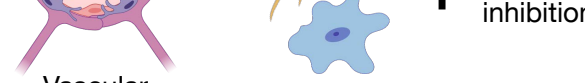

Vascular

niche

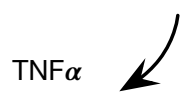

IL6

ROS/NO

MMP14

IL4
GO-terms (Fig. 6c+Ext. Fig. 6f).

- Response to CSF2RB and IL4

- STAT5 targets

- MMP14 correlated

- (Cellular) response to oxygen containing compound

- (Positive) regulation of TNF superfamily cytokine production

- (Cellular) response to IL6

- Cellular response to IL4 


\section{Extended data Fig. 7 CSF2 protects TAMs from BLZ945 induced killing.}

a, Quantification of the cell viability of BMDM and EOC2 MG cells in response to CSF2 and/or IL4 stimulation in the presence or absence of 67nM and 670nM BLZ945 ( $n=4-8$ replicates per condition). b, Western blot analysis of the CSF-2R downstream signaling effector STAT5 in response to cytokines or tumor cell-conditioned media (Tu-CM) stimulation in the presence or absence of 670nM BLZ945 in BMDMs and EOC2. Result shown is representative of three independent experiments. c, Gene expression of CSF2/Csf2, CSF2RA/Csf2ra and CSF2RB/Csf2rb in FACS purified MDA-BrM tumor cells, TAM-MG and TAM-MDM as well as normal MG and blood monocytes and $M G(n=5$; tumor-bearing mice and $n=3$; tumor-free controls). d, Model summarizing the findings from this study in comparison to results from models of neuro-inflammation. In neuro-inflammation, B- and T-cells as well as endothelial cells and astrocytes are known sources for CSF2. CSF2-activated CCR2+ monocytes are the predominant cell type in EAE models to secrete effector molecules that lead to neurotoxic tissue damage. CSF2-activated TAMs in BrM displayed gene signatures that indicate the activation of similar effector molecules (TNF, IL6, ROS/NO and MMP14) based on the listed GO terms we uncovered herein. 


\section{Extended data Fig. 8 CSF1R and STAT5 inhibition in vitro and in vivo.}

a, Schematic overview of CSF1R and CSF2Rb downstream signaling. Molecular targets inhibited in this study (in preclinical models and in cell culture) are indicated. b, Quantification of cell viability in BMDM and EOC2 MG cells in response to increasing concentrations of neutralizing CSF2Rb antibody by MTS assay ( $n=4$ replicates per condition). c, Quantification of cell viability in BMDM and EOC2 cells in response to increasing concentrations of the STAT5 inhibitor AC4-130 by MTS assay ( $n=4-12$ replicates per condition). $\mathbf{d}$, Western blot analysis of the CSF2R downstream signaling pathway STAT5 in response to CSF2 and increasing concentrations of AC4-130 in the presence or absence of 670nM BLZ945. Result shown is representative of three independent experiments. e, Quantification of the relative tumor growth rate based on volumetric evaluation of MRI images in the MDA-BrM model $(n=8$; vehicle, $n=10 ; B L Z 945, n=6 ; A C 4-130, n=8 ; B L Z 945+A C 4130)$. Data is represented as individual replicates with lines depicting the mean of each experimental group. Analyzed by two-tailed Students t-test based on area under the curve. $f$, Representative images and 3D reconstruction of IBA1+ cells in the MDA-BrM model depicting the morphology of TAMs in intra- and peri-tumoral areas as well as normal adjacent parenchyma. Scale bars, $100 \mu \mathrm{m}$ and $25 \mu \mathrm{m}$. 